\title{
In- and outpatient lifestyle interventions on diet and exercise and their effect on physical and psychological health: a systematic review and meta-analysis of randomised controlled trials in patients with schizophrenia spectrum disorders and first episode of psychosis
}

\author{
Authors: \\ Fernández-Abascal, Blanca ${ }^{a^{*}+}$ \\ Suárez-Pinilla, Paula ${ }^{a, \mathrm{e}^{* *+}}$ \\ Cobo-Corrales, Carlos ${ }^{\mathrm{c}}$ \\ Crespo-Facorro, Benedicto d, e \\ Suarez-Pinilla, Marta ${ }^{b+}$
}

a Department of Psychiatry, University Hospital Marqués de Valdecilla, IDIVAL, Santander, 39011, Spain

${ }^{b}$ Department of Neurodegenerative Disease, Institute of Neurology, University College of London, London, WC1N 3AX, UK

c School of Education, University of Cantabria, Santander, 39005, Spain

d Department of Psychiatry, School of Medicine, University Hospital Virgen del Rocío - IBiS, Sevilla, 41013, Spain

e Centro de Investigación Biomédica en Red de Salud Mental (CIBERSAM), Instituto de Salud Carlos III, Madrid, 28029, Spain

+ B F-A., M S-P. and P S-P. contributed equally to this work

*Corresponding author: Blanca Fernández-Abascal. Department of Psychiatry, University Hospital Marqués de Valdecilla. Avda Valdecilla s/n, 39008, Santander, Spain. Phone: +34-65097-90-88. E-mail: blancafap@hotmail.com 


\begin{abstract}
Patients with non-affective psychosis often lead unhealthy lifestyles. We performed a systematic review and meta-analysis on non-pharmacological RCTs for improvement of diet and physical activity in non-affective psychosis patients, including first-episode psychosis. A variety of outcomes was analysed, including metabolic, psychopathology, cognitive, functional and quality of life outcomes. Fifty-nine studies were included. An improvement in anthropometric measurements (BMI, weight, waist circumference) was observed post-intervention, persisting after follow-up. Post-intervention benefit was found also for psychotic symptoms severity (also persisting after follow-up), many cognitive domains and physical and global functioning and quality of life. Conversely, no effect was observed in relation to most blood metabolites, blood pressure and non-psychotic psychopathology and spontaneous physical activity. Improvement was generally larger for interventions including exercise, especially moderate/vigorous aerobic exercise, but follow-up maintenance was greater for psychotherapy interventions. Sensitivity analyses limited to chronic stages of psychosis and low risk of bias studies produced comparable results. Further studies are needed to design optimized interventions in this vulnerable population.
\end{abstract}

Key words: non-affective psychosis; schizophrenia; non-pharmacological interventions; physical activity; exercise; psychoeducation; health promotion; cardiometabolic risk; quality of life; meta-analysis. 


\section{HIGHLIGHTS}

- Non-pharmacological interventions improve many outcomes in non-affective psychosis.

- Effect is observed on cognitive, clinical and functional and life-quality outcomes.

- Benefit is larger in exercise interventions, especially moderate aerobic exercise

- Effect lasts after follow-up on anthropometric outcomes especially in psychotherapy

- Benefit is observed also specifically in chronic stages of non-affective psychosis 


\section{Introduction}

Persons with Schizophrenia Spectrum Disorders (SSD) have a high prevalence of obesity, unfavourable cardiometabolic parameters and therefore, a higher probability to develop physical health problems (De Hert et al., 2011). The mechanisms underlying these metabolic disturbances are complex and involve an interaction of genetic and external factors including lifestyle and environmental conditions (Henderson et al., 2015). External contributing factors playing an important part include unhealthy diet habits, smoking, sedentary behaviour and often living in more deprived environments due to poor social and labour functioning (Gage et al., 2016; Ratliff et al., 2012). In addition, the most effective second-generation antipsychotics, such as clozapine or olanzapine, have important metabolic side-effects, leading to weight gain and metabolic impairments (Cooper et al., 2016; Leucht et al., 2013), moreover, for some drugs, higher doses might not provide more efficacy but increase secondary effects (Leucht et al., 2020). For all of this, persons with SSD have higher prevalence of metabolic syndrome, with older age and illness duration exerting the strongest influence on the rate of metabolic syndrome (Mitchell et al., 2013). As consequence of obesity, metabolic disturbances and cardiovascular diseases, SSD patients has a life expectancy about 15-20 years shorter than the general population (Newcomer and Hennekens, 2007).

Moreover, quality of life (QoL) in persons with schizophrenia (SCZ) and related disorders is often worse than that of the general population and that of other physically ill patients; negative and depressive psychopathology correlates inversely with QoL, and is often associated with social and occupational dysfunction (Bobes et al., 2007). In both, general and SSD population, decreased physical activity has been associated with metabolic disturbances but also with worse psychosocial functioning and QoL (Ohi et al., 2019). Specifically, low exercise has been related to more negative symptomatology, poorer independence and motivational performance, and lower occupational and psychosocial functioning in patients with psychosis (Vancampfort et al., 2012a). In order to tackle these problems, it seems essential to implement non-pharmacological interventions promoting exercise or healthy lifestyles in patients with serious mental illness. Among non-pharmacological approaches, programs using physical activity, psychotherapies such as psychoeducation, behavioural counselling, motivational interviewing or cognitive behavioural therapy based on lifestyle interventions, or a combination of techniques, have been tested. Pharmacological approaches using topiramate, metformin, statins or switching to a more cardio-health agent were tested when the main objective is to improve physical health as a short-term outcome (Vancampfort et al., 2019). When results of different approaches are taken together, a major issue is the lack of randomization or the absence of control groups (Tumiel et al., 2019). Thus, a common form of assessing the effectivity of these interventions is through randomised controlled trials (RCT), with evaluation of the variables of interest (e.g., metabolic, cognitive, psychosocial functioning-related variables) in both the experimental and the control group, before and after the intervention. Occasionally, these participants are followed for a period after the intervention in order to assess its long-term effectivity.

These interventions have had conflicting results about their effectivity. While many studies have reported improvement of metabolic parameters (weight, glucose, lipidic profile, etc), clinical 
symptomatology and functioning at the end of the intervention, the long-term persistence of such improvement is more doubtful (Caemmerer et al., 2012). A recent meta-review of metaanalyses shows that lifestyle counselling and exercise were the most effective interventions for weight reduction (Vancampfort et al., 2019). In the same vein, exercise and dietary counselling in conjunction with behavioural therapy should be firstly considered for weight reduction in SCZ (Faulkner et al., 2003). Regarding clinical and functional outcomes, cognitive behavioural therapy has a modest therapeutic effect on QoL in individuals diagnosed with SSD at end-oftrial, although this benefit is not evident at follow-up (Laws et al., 2018). Moreover, in patients with psychosis, interventions could significantly reduced negative symptoms when were focused on aerobic exercise (AE) (Sabe et al., 2020) or alternative forms of activity such as yoga or tai-chi (Vogel et al., 2019) and mindfulness based approaches (Sabe et al., 2019). Mind-body exercises (MBE) also seemed to improved patients' QoL and mental health (Vera-Garcia et al., 2015). As add-on treatments, yoga therapy reduced general psychopathology in patients with SCZ (Vancampfort et al., 2012b); and progressive muscle relaxation reduced state anxiety and psychological distress (Vancampfort et al., 2013).

Recent meta-analyses and systematic reviews about healthy-life interventions in schizophrenia have approached the study of the optimal characteristics of the intervention, such as its type (exercise, psychotherapy or a combination), intensity, duration, setting (inpatient/outpatient), treatment approach (group/individual therapy) and population target. In regard to physical activity, a systematic review recommend a frequency of at least three times a week during a minimum of 12 weeks among patients with SCZ (Stanton and Happell, 2014), while others show a modest but significant weight loss or improved cardiovascular fitness independently of the length of intervention (more or less than 12 weeks (Tumiel et al., 2019). However, as the lifestyle change needs to be permanent, most of authors state that interventions should last at least around six months (Sabe et al., 2020) and should provide follow-ups consisting in booster sessions for behavioural control of diet and activity (Bonfioli et al., 2012). Concerning to the duration of exercise, emerging data suggest that symptomatic or functional benefit in this population is observed with 150 minutes of moderate-vigorous physical activity per week (Ashdown-Franks et al., 2020; Ohi et al., 2019) or 30 min of $\mathrm{AE}$ at a minimum of $50 \%$ peak $\mathrm{O}_{2}$ consumption $\left(\mathrm{VO}_{2} \max \right)$ for each session (Sabe et al., 2020), while group approaches may enhance treatment adherence (Vogel et al., 2019). Moreover, it is possible that the effectivity of these programs may be higher in patients with first episode of psychosis (FEP) or relatively newly diagnosed SSD, as these patients have still relatively preserved cognitive and psychological functioning and may be more suitable for lifestyle intervention (Gates et al., 2015).

To date, many authors have been devoted to the study of the efficacy of non-pharmacological interventions in psychiatric population through review of previous RCTs. These interventions are focused in promoting healthy lifestyle changes in an active way (e.g., exercise intervention) or indirectly, through psychotherapy, and also by a combination of both. Moreover, the effect of non-pharmacological interventions on psychological and functional areas has been relatively less investigated than its effect on metabolic, cardiorespiratory and physical parameters (Gurusamy et al., 2018; Ohi et al., 2019). In regard to the setting, the majority of available RCTs are based on the community field, but recently, there are efforts to specifically explore the inpatient setting, and differences have been found in the baseline level of physical activity level 
(Ohi et al., 2019; Stubbs et al., 2016). Several studies have focused on serious mental illness settings, including major depression disorder, bipolar disorder and SSD as a whole (Tosh et al., 2011) or separately (Stubbs et al., 2018); others have evaluated studies based on patients taking antipsychotics for non-affective but also affective psychosis (Mucheru et al., 2019) and only a few contemplate samples of FEP patients (Gates et al., 2015). Nevertheless, studies based on SSD patients constitute a special subset because of their particular characteristics since SCZ is a severe, disabling, lifelong disorder, associated with severe social and occupational dysfunction (Bobes et al., 2007).

Therefore, the aim of this review is to examine the available evidence from RCTs on different non-pharmacological interventions in patients with SSD and/or FEP compared with controls, in order to systematically assess the effectiveness of such interventions in terms of improving physical and psychological health and well-being. We also aim to explore whether effectiveness depends on some of the abovementioned characteristics (e.g., patient diagnosis, setting, type of intervention, duration, etc) as well as whether the effects, if any, persist in the long term after the end of the intervention. 


\section{Material and methods}

The protocol for this review was previously registered on PROSPERO (registration CRD42020153408); and the PRISMA guidelines (Preferred Reporting Items for Systematic Reviews and Meta-Analysis) were followed to report the results (Moher et al., 2009).

\subsection{Comprehensive search of literature}

A comprehensive search in English and Spanish was conducted through the electronic databases Pubmed, Embase and the Cochrane Central Register of Controlled Trials. Scientific articles published in both English and Spanish before April 2020 were considered for inclusion. The employed key search terms, extracted from MeSH, were as follows: "psychotic disorders OR schizophrenia OR schizophrenia spectrum and other psychotic disorders" AND "metabolic syndrome OR cholesterol OR body weight OR body mass index OR waist circumference OR triglycerides OR blood pressure OR arterial pressure OR neuropsychological tests OR behavioral symptoms OR mental health OR quality of life" AND "weight reduction programs OR exercise OR health education OR health promotion OR cognitive behavioral therapy". Reference lists from the identified articles and previous reviews were also explored.

\subsection{Eligibility and study selection}

No time restriction was considered for the search. Studies were selected if they met the following criteria: 1) Randomised controlled trials (RCTs) evaluating the effect of nonpharmacological protocolized interventions based on exercise and/or psychotherapy focused on changes in diet and physical activity, aiming to promote physical health, psychological wellbeing and/or changes in lifestyle in patients with schizophrenia-spectrum disorder (SSD) or first episode of psychosis (FEP). SSD encompassed ICD-10 codes F20-F29: schizophrenia (SCZ), schizotypal, delusional, and other non-mood psychotic disorders. Interventions consisting on yoga or tai-chi were included as mind-body exercises (MBE). Psychotherapies that were not specifically designed to induce changes in diet and physical activity were not considered -such as meditation or cognitive remediation; 2) Reporting any results of anthropometric measures and metabolic parameters, and/or clinical and psychopathological outcomes, and/or results related to quality of life and functioning; 3 ) Reporting their results qualitatively or quantitatively; 4) Studies were carried-out on inpatient and outpatient settings; 5) Studies had a control group constituted by patients with SSD/FEP that did not perform the experimental intervention; thus, those trials comparing two different populations (e.g. patients versus healthy controls) were not eligible; 6) Studies were eligible if the comparison group had treatment as usual, defined as the standard care in that setting, waiting-list, or any non-protocolized non-active intervention (e.g. casual counselling); thus, RCTs comparing two different active interventions (e.g. yoga and jogging) were not included. Non-RCT studies, reviews, meta-analyses, case series, case reports, books, comments, conference papers, editorials and animal research articles were also excluded. 
When articles comprised the results of the follow-up of a previous published RCT, they were also considered for this review if the index RCT meet the inclusion criteria. Follow-up studies of previous RCT were excluded when any group, experimental or control, performed the active intervention during the follow-up period.

Two reviewers ( $F-A, B$ and $S-P, P)$ searched the literature independently and determined the eligible studies after examining title and abstract. Full text was examined for each potentially eligible article. Discrepancies over included articles were addressed through discusions with a third reviewer $(S-P, M)$.

\subsection{Study quality and risk of bias}

Risk of bias (RoB) was assessed for each included study using the Cochrane Collaboration Risk of Bias Tool (Higgins et al., 2011) for the following domains: sequence generation, allocation concealment, masking of outcome assessors, incomplete outcome data and selective reporting. For each domain, studies were clasified in "high risk", "low risk" or "unclear risk" of bias. Unclear risk of bias was used when the RCT did not provide sufficient details for the specific domain. The total score ranges from 1 to 5 , with higher scores meaning less risk of bias. Two reviewers ( $F-A, B$ and $\mathrm{S}-\mathrm{P}, \mathrm{P})$ assessed independently the risk of bias and any discrepancies were solved through discussion with a third reviewer (S-P, M).

\subsection{Data extraction}

Data were extracted in duplicate by two independent reviewers (F-A,B and S-P,P). Disagreements were resolved by discusion with a third reviewer (S-P,M). A pre-defined database was created to extract the data for each study containing the following variables: 1$)$ Study: authors, country and year; 2) Patient's characteristics: age, gender, diagnosis (SCZ only, FEP, FEP+SSD, SSD includying SCZ), antipsychotic medication for each comparison group (percentage of patients taking clozapine/olanzapine, as they are associated with the highest risk of metabolic disturbances and weight gain (Allison and Casey, 2001; Cooper et al., 2016; Leucht et al., 2013); 3) Sample size of each randomised group; 4) Type of experimental intervention (physical exercise, psychotherapy or combined intervention including both, psychotherapy and exercise). Based on previous meta-analyses of physical activity in patients with schizophrenia, interventions based on exercise were grouped into $\mathrm{MBE}$, (including yoga and tai-chi) and physical exercises (Vogel et al., 2019); furthermore, physical exercise was divided into aerobic exercise (AE) and anaerobic exercise (AnE) (Sabe et al., 2020) Aerobic exercise was in turn subdivided in light $A E$-when the greatest part of the activity consisted of low consumption of energy such as walking or house-working-, and moderate/vigorous AE - if there was a high energy consumption for more than 5 minutes, such as running or dancing- . Therefore, there were five possible subtypes of physical exercise: Light $A E$, Moderate/vigorous $A E, A n E, M B E$, and mixed exercises (combinations of two of the previous types, such as light $A E+A n E$ or lightAE+MBE). As 
for psychotherapy interventions, they were classified into three subtypes: psychoeducation including structured counselling, psychoeducation and motivational interview; cognitivebehavioural therapy (CBT) -encopassing structured cognitive and/or behavioral therapies- and mixed psychotherapies (including both, psychoeducation and CBT) (Bechdolf et al., 2004). Interventions were named as "combined therapies" if they used both, exercise and psychotherapy; 5) Frequency and duration of intervention, as well as total number of hours of engagement; 6) Fetting (in vs outpatient); 7) mode (individual vs group format); 8) Type of control intervention; 9) Drop-outs; 10) Outcomes: means and measures of dispersion of anthropometric measures and metabolic parameters before and after intervention (body weight and body mass index (BMI), glucose, cholesterol, triglycerides, blood pressure (BP), and waist circumference (WC)); means and measures of dispersion of psychopathological and clinical variables before and after intervention; means and measures of dispersion of functionallity scores and QoL assessments before and after intervention; 11) Follow-up (when available): length and outcome values after the follow-up.

When studies included some patients with diagnoses other than the purpose of this review, but results were reported separately by diagnosis group, only those results of patients with SSD or FEP were included in the qualitative and quantitative synthesis. Likewise, when studies employed different types of active intervention (e.g. pharmacological and nonpharmacological), only those intervention groups that met our criteria were considered.

\subsection{Data analysis}

A meta-analysis on the results of included studies was performed for all available outcomes (reported in at least 2 studies) related to anthropometric measures, metabolic parameters, psychopathological and clinical symptoms, cognitive tests, functionality and QoL scores. Each outcome was analysed separately. When different scores were considered to measure the same theoretical construct (e.g., roughly equivalent QoL scales) they were pooled in a single analysis. When a single study examined more than two active treatments (e.g., aerobic exercise and yoga, each compared with a third control group), the effect size for each intervention was included independently. In those cases wherein a meta-analysis could not be performed, a summary of the qualitative results is reported.

The considered effect size was standardized mean difference (Hedges' g) of the change in the outcome variable between the intervention and control group, measured before and after the intervention, and when available, before intervention and the end of follow-up. A secondary meta-analysis based on non-standardized mean difference was also performed, if the form of the data permitted it.

Quantitative synthesis was possible when studies reported: 1) the summary effect size for the difference between groups in any manner that could be converted into standardized mean difference with its standard error; 2) mean and dispersion statistics of the changes in the outcome variable (pre/post intervention, and when available, pre-intervention/ end of followup) separated into intervention and control groups; 3 ) mean and dispersion statistics (or else 
full data for all participants) of the outcome variable before and after intervention (and at the end of follow-up when available), separated into intervention and control groups.

Effect sizes from individual studies were pooled by the DerSimonian and Laird random-effect model (DerSimonian and Laird, 2015). Between-study heterogeneity was investigated by visual inspection of the forest plots and calculation of the Cochrane's $Q, \tau^{2}$ and $I^{2}$ statistics (Cochran, 1954; Higgins et al., 2003; Sterne et al., 2008) .

Effect sizes in the Results section are described following the conventions suggested by Cohen and Sawilowsky, i.e., 'very small' for effects below 0.2, 'small' between 0.2 and 0.5, 'medium'/ 'moderate' for 0.5-0.8, 'large' for 0.8 - 1.2 and 'very large' for effects larger than 1.2, regardless of sign (Cohen, 1998; Sawilowsky, 2009). Likewise, the amount of heterogeneity was described in terms suggested by the Cochrane Handbook for Systematic Reviews of Interventions (Higgins et al., 2019)

Subgroup analyses were performed for exploring potential sources of heterogeneity in the effect size based on the type of intervention (exercise, psychotherapy, combined), subtype of intervention (Light $A E$, Moderate/vigorous $A E, M B E, A n E$, mixed exercises; psychoeducation, CBT, mixed psychotherapies; combined exercise + psychotherapy), mode of intervention (group or individual format), diagnosis (SCZ, SSD, FEP, FEP+SSD) and setting (in or outpatient).

To further ascertain the effectivity of intervention in chronic stages of psychosis, sensitivity analyses were performed on two study subsets: 1 ) all studies not including FEP patients (i.e., SCZ and SSD studies) and 2) all studies not including FEP patients and explicitly reporting an average time since diagnosis $\geq 5$ years).

Sensitivity analyses were also conducted on studies classified as low risk of bias (low-RoB) studies, defined as having $\geq 4$ points on the Cochrane Collaboration Risk of Bias Tool. For both, chronic stages of psychosis and low-RoB studies, sensitivity analyses involved calculation of overall and subgroup-based effect sizes in the defined subsets. The results of these analyses are only reported for those outcomes wherein they sensibly differ from results computed on the entire sample, but forest plots for all outcomes are presented in the Supplementary Materials regarding chronic psychosis, plots are provided for the first subset only.

Random-effects meta-regression was performed in order to formally ascertain the association between the effect size of the individual studies and specific characteristics of the intervention, namely setting, type of intervention and total hours of engagement (weekly hours multiplied by duration). In order to prevent spurious findings (Higgins and Thompson, 2004), meta-regressions were limited to the subsets of low-RoB studies and only performed on those outcomes where the study sample was $\geq 10$. Furthermore, to reduce the risk of false positives derived from multiple comparisons, the number of predictors was limited to those outlined above and $p$ values were adjusted by the Monte Carlo permutation method. Study-level averages of individual characteristics (i.e., mean initial BMI, mean age, etc) were not considered as they would be liable to ecological fallacy (where study-level associations may not apply to individuals). Meta-regression results are only reported in the Results section for those outcomes where at least borderline associations $(p<0.10)$ were found. 
Publication bias was assessed by visual inspection of the funnel plot and Egger's test for smallstudy effects. In presence of funnel plot asymmetry, contour-enhanced funnel plots were built with $90 \%, 95 \%$ and $99 \%$ levels of statistical significance, to better distinguish between outlying effect sizes in small studies and actual publication bias (where asymmetry would be mainly found in the non-significant regions)(Peters et al., 2008). These analyses are only detailed in the Results section for those outcomes where small-study effects were suspected by any of the aforementioned tests: in such cases, nonparametric trim-and-fill method was applied in order to correct the observed effect size by imputation of (purported) missing studies in case of publication bias. Funnel plots for all outcomes are provided as Supplementary Materials.

Unless stated otherwise, reported analyses pertain to standardized effect size (Hedges' g) and reported intervals are $95 \%$ confidence intervals $(95 \% \mathrm{Cl})$. However, for the overall analysis on each outcome variable (but not for subgroup analysis), non-standardized mean difference (WMD) is also explicitly reported, and the $95 \%$ prediction intervals (95\% P.I.) for the standardized mean difference are reported alongside confidence intervals, except for cases when these measures are not suitable. In order to prevent false positive findings in relation to the multiple tested outcomes, the cut-off point for statistical significance in the overall analyses was corrected from the conventional value $\mathrm{p}=0.05$ by Benjamini-Hochberg false discovery rate (FDR) controlling procedure.

All analyses were performed using Stata 13 (Stata Corp, College Station Tex., USA), with metan, metareg, metafunnel and metabias commands. 


\section{Results}

The process of study selection is shown in figure 1. Out of the 3089 articles encountered, 733 were selected after removal of duplicates. Following exclusion of articles based on reading title and abstract, full texts for the remaining 185 studies were assessed for eligibility; among these, 125 studies were excluded for different reasons detailed in figure 1. Eventually, 60 studies were included for the qualitative synthesis $(57$ reporting experimental interventions and three followup studies published separately); among those 60, 55 studies were included for meta-analysis.

\subsection{Description of included studies}

Data from included studies are summarized in table 1 and a synthesis of antipsychotic treatments used for each study is given in supplementary table 1.

Studies were divided into two blocks according to setting: in versus outpatient studies. These blocks were in turn split in three subgroups according to their experimental arm: any type of physical exercise (including yoga and tai-chi), any psychotherapy, and both interventions combined (physical exercise plus psychotherapy). Overall, there were 13 inpatient and 47 outpatient studies (including three follow-up-only studies). Within the inpatient subgroup, 9 were exercise-based interventions (one included follow-up), one employed psychotherapy (with follow-up data) and three implemented a combination of the two; with regards to diagnosis, 11 enrolled participants with SCZ only, one included any SSD diagnosis, and one a mixed sample of FEP + SSD patients. As for the outpatient subgroup, 22 ascertained the effect of exercise (with follow-up in five cases), 18 used psychotherapy (eight with a follow-up) and 4 a combined intervention; regarding diagnosis, 18 recruited SCZ only, 20 enrolled any SSD patients, 4 FEP patients only and 2 any of the previous (FEP + SSD). Out of the 60 included articles, 59 were written in English and 1 in Spanish.

\subsection{Quality assessment}

The methodological quality assessment of each included study was performed according to the Cochrane Collaboration Risk of Bias Tool (table 2). A low risk of bias was found in 53/60 for random sequence generation, $27 / 60$ for allocation concealment, $41 / 60$ for reporting bias. 40/60 for detection and 60/60 for attrition bias. The number of studies with unclear risk of bias was considerable in two domains: 26/60 for allocation concealment and 12/60 for detection bias, whereas it was small for random sequence generation (6/60), reporting bias (9/60) and for attrition bias (7/60). Twenty-three studies were considered to have high risk of bias in any domain: 160 for random sequence generation, 7/60 for allocation concealment, 10/60 for reporting bias, $8 / 60$ for detection bias and $3 / 60$ for the attrition domain.

\subsection{Anthropometric measures and metabolic outcomes}




\subsubsection{Body mass index (BMI)}

\subsubsection{BMI change at the end of intervention}

Twenty-six studies examined the effect on BMI change at the end of the intervention (ÁlvarezJiménez et al., 2006; Armstrong et al., 2016; Attux et al., 2013; Battaglia et al., 2013; Beebe et al., 2005; Brar et al., 2005; Cheng et al., 2017; Cordes et al., 2014; Gaitero-Calleja et al., 2007; Holt et al., 2019; Iglesias-García et al., 2010; Kaltsatou et al., 2015; Kwon et al., 2006; Littrell et al., 2003; Lovell et al., 2014; Marzolini et al., 2009; McCreadie et al., 2005; McKibbin et al., 2006; Methapatara and Srisurapanont, 2011; T. W. Scheewe et al., 2013; Speyer et al., 2016; Sugawara et al., 2018; Svatkova et al., 2015; Weber and Wyne, 2006; Wu et al., 2007, 2008). Twenty-four could be pooled for meta-analysis (figure 2a).

The effect size in favour of intervention was borderline large (Cohen, 1998): D+L Hedges'g = $0.797(-1.102,-0.492), p<0.001$. In terms of non-standardized mean difference (including 23 studies as this result was not available for one), the intervention entailed an overall decrease of WMD $=-0.883\left(\mathrm{~kg} / \mathrm{m}^{2}\right), 95 \% \mathrm{Cl}(-1.359-0.407), \mathrm{p}<0.001$. All further analyses pertain to standardized effect size; note that a more negative effect size is more in favour of intervention in the case of BMI change (greater BMI reduction in the intervention compared to control group).

There was considerable between-study heterogeneity: $Q^{2}=208.23(\mathrm{df}=23, p<0.001) ; \tau^{2}=0.4495$; $I^{2}=89.00 \%$. As a result, even though the $95 \%$ confidence intervals for Hedges' $\mathrm{g}$ were well below 0 -indicating that the average effect size obtained by random-effects pooling was significantly in favour of intervention-, the $95 \%$ prediction intervals did contain zero (95\% P.I. $(-2.22,0.63)$, indicating that some individual studies would be expected not to favour intervention.

\subsection{Subgroup analyses}

We aimed to explore the sources of this heterogeneity by subgroup. Splitting studies by setting (figure 2a), a larger effect size was observed, on average, for inpatient ( $N=4, g_{\text {inpatient }}=-1.479$ ($\left.2.587,-0.370), \mathrm{I}^{2}=92.1 \%\right)$ compared to outpatient setting $(\mathrm{N}=20$, goutpatient $=-0.689(-1.006,-$ $\left.0.371), I^{2}=88.4 \%\right)$. However, there was considerable heterogeneity within both subgroups, which affects the validity of the between-subgroup comparison.

Subgroup division by broad type of intervention (exercise, psychotherapy, combined) showed that combined therapies (exercise + psychotherapy) had the largest average effect size ( $\mathrm{N}=3$, $\left.\mathrm{g}_{\text {combined }}=-2.752(-5.227,-0.277), 1^{2}=97.3 \%\right)$, exercise-based therapies ranked second $(\mathrm{N}=7$, $\left.g_{\text {exercise }}=-0.906(-1.969,0.157), p=0.095,1^{2}=89.4 \%\right)$, and psychotherapy had the lowest effect size (but statistically significant: $\left.\mathrm{N}=14, \mathrm{~g}_{\text {psychotherapy }}=-0.449(-0.671,-0.228), \mathrm{I}^{2}=73.0 \%\right)$. Note that there was still considerable heterogeneity within the exercise and combined subgroups.

Examining specific subtypes of exercise interventions, moderate/vigorous aerobic exercise (AE) had large effect size $\left(\mathrm{N}=4, \mathrm{~g}=-1.685(-3.262,-0.108), \mathrm{I}^{2}=93.2 \%\right)$, whereas no effect was found for mixed aerobic/anaerobic (AE/AnE) programs $\left(N=3, g=-0.003(-1.004,0.998), I^{2}=79.9 \%\right)$. Regarding psychotherapies, small effect size was reported both for psychoeducation $(\mathrm{N}=4, \mathrm{~g}=-$ $\left.0.443(-0.814,-0.073), I^{2}=60.3 \%\right)$ and cognitive behavioral therapy (CBT) $(N=6, g=-0.320(-0.564$, $-0.076), I^{2}=56.3 \%$ ). However, mixed psychotherapy (combining psychoeducation and CBT) was associated to large effect size $\left(\mathrm{N}=4, \mathrm{~g}_{\mathrm{MPs}}=-0.860(-1.622,-0.099), \mathrm{I}^{2}=86.6 \%\right)$. 
Considering mode of intervention, the effect size for group-based approaches was large $(\mathrm{N}=18$, $\left.\mathrm{g}_{\text {group }}=-1.021(-1.466,-0.577), I^{2}=91.4 \%\right)$, whereas individual interventions had small effect size $\left(N=6, g_{\text {indiv }}=-0.427(-0.679,-0.176), I^{2}=55.5 \%\right)$.

Splitting studies by diagnosis, the two studies enrolling FEP patients (Álvarez-Jiménez et al., 2006; Wu et al., 2008) had the largest average effect size compared to other diagnostic categories, but with considerable within-subgroup heterogeneity $\left(N=2, g_{\text {FEP }}=-3.656(-9.557\right.$, 2.244), $\left.I^{2}=98.6 \%\right)$. Sensitivity analyses for the effectivity of intervention in chronic stage of disease produced results largely comparable to the entire dataset (see Supplementary Materials).

\subsection{Analyses on studies with low risk of bias}

Sensitivity analyses were conducted including only studies with low risk of bias (RoB) $(N=18)$. Overall and subgroup-based results were comparable in size and statistical significance - see Supplementary Materials.

Random-effects meta-regression was performed on these low-bias studies to further explore the influence of setting, type and total hours of intervention: no association was found to effectivity.

As mentioned in the Data analysis section of Material and Methods, sensitivity analyses for chronic psychosis and low-RoB studies were performed for all outcomes but henceforth reported in the main text only if they sensibly differed from the entire sample. Likewise, metaregression as described for $\mathrm{BMI}$ was performed for all outcomes with at least 10 studies, but only reported in light of (near) significant associations.

\subsection{Publication bias}

The funnel plot was highly asymmetrical as, confirmed by Egger's test for small-study effect $(p=0.002)$. However, further inspection with enhanced significance contours indicated that this was due to small studies with highly-significant effect sizes, unlikely to arise by publication bias (see Supplementary Materials for this and all funnel plots). Indeed, non-parametric adjustment by trim-and-fill imputation method yielded identical effect size to the original, with no studies imputed.

Two (of 26) studies were not included in the meta-analysis as the effect size could not be calculated: one with exercise therapy in SCZ outpatients (Beebe et al., 2005) and another about psychotherapy based on diet advice in SCZ outpatients (McCreadie et al., 2005). Neither of the two reported any significant $\mathrm{BMI}$ change.

\subsubsection{BMl change at the end of follow-up}

Out of the 26 studies on BMI change, only seven examined the potential persistence of the effect after follow-up (Álvarez-Jiménez et al., 2010; Cheng et al., 2017; Cordes et al., 2014; GaiteroCalleja et al., 2007; Littrell et al., 2003; McCreadie et al., 2005; McKibbin et al., 2010). Out of these, five were suitable for meta-analysis, with an average duration of 30.2 weeks since the end of the intervention and 47.2 since the beginning of experiment. The change in outcome variable (BMI) was assessed between end of follow-up and before the start of intervention.

The effect was medium-sized (figure 2b): D+L Hedges' $g=-0.570,95 \% \mathrm{Cl}(-0.916,-0.224) \mathrm{p}=$ $0.001,95 \%$ P.I. $(-1.60,0.46)$. In terms of non-standardized mean difference: $W M D=-1.087 \mathrm{~kg} / \mathrm{m} 2$ 
(95\% Cl $-1.804,-0.371) p=0.003)$. There was no significant between-study heterogeneity: $Q^{2}=$ $7.61(d f=4, p=0.107), \tau^{2}=0.0733, l^{2}=47.40 \%$.

Subgroup division into in and outpatient settings showed no clear differences (although the only inpatient-based study had the largest effect size of all five studies). In terms of type of intervention, while no significant long-term effect was observed for the single exercise-based study $(\mathrm{N}=1$, gexercise $=-0.031(-0.599,0.537))$, there was medium effect size for psychotherapy $\left(\mathrm{N}=4, \mathrm{~g}_{\text {psychotherapy }}=-0.694(-0.989,-0.398), \mathrm{I}^{2}=12-3 \%\right.$. Further examination on specific subtypes of exercise and psychotherapy showed that studies based on psychoeducation $(\mathrm{N}=1)$ and mixed psychotherapy ( $N=2)$ had significant long-term effect, whereas moderate/vigorous $A E$ and $C B T$ did not ( $\mathrm{N}=1$ each). No difference was established between individual and group therapies.

Regarding patient's diagnosis, subgroup splitting showed that SSD was associated to significant, large effect size $\left(\mathrm{N}=3, \mathrm{~g}_{S \mathrm{SD}}=-0.856,95 \% \mathrm{Cl}(-1.184,-0.528), \mathrm{I}^{2}=0 \%\right.$; by contrast, no significant effect was encountered for FEP or SCZ patients ( $\mathrm{N}=1$ each).

Two studies: one about psychotherapy in SCZ outpatients (McCreadie et al., 2005) and other with combined intervention in SSD outpatients (Gaitero-Calleja et al., 2007) with a follow-up of 52 and 12 weeks, respectively, did not provide numeric effect sizes but reported no effect on BMI.

\subsubsection{Body weight}

\subsubsection{Body weight change at the end of intervention}

There were twenty-five studies examining change in body weight (Álvarez-Jiménez et al., 2006; Armstrong et al., 2016; Attux et al., 2013; Battaglia et al., 2013; Brar et al., 2005; Brown et al., 2014; Cheng et al., 2017; Cordes et al., 2014; Fisher et al., 2020; Holt et al., 2019; Hsu et al., 2016; Iglesias-García et al., 2010; Jean-Baptiste et al., 2007; Kwon et al., 2006; Littrell et al., 2003; Lovell et al., 2014; Marzolini et al., 2009; McKibbin et al., 2006; Methapatara and Srisurapanont, 2011; Rotatori et al., 1980; T. W. Scheewe et al., 2013; Scocco et al., 2006; Speyer et al., 2016; Sugawara et al., 2018; Weber and Wyne, 2006; Wu et al., 2007, 2008); out of these, twenty-four reported results in a manner suitable for meta-analysis.

At the end of intervention, the experimental group had a weight reduction of $-2.121 \mathrm{~kg}(95 \% \mathrm{Cl}$ $-3.067,-1.176) p<0.001$, compared to controls. In terms of standardized mean difference, the effect in favour of intervention was medium-sized: $g=-0.528(95 \% \mathrm{Cl}-0.729,-0.327) p<0.001$, with $95 \%$ P.I. $(-1.38,0.33)$. There was substantial between-study heterogeneity: $Q^{2}=86.08$ $(d f=24, p<0.001), \tau^{2}=0.1601, l^{2}=72.1 \%$. See figure $3 a$.

Subgroup analysis by patient setting did not show a noticeable difference in average effect, which was medium-sized and statistically significant in both inpatient and outpatient setting.

In terms of type of intervention, the average effect size for the combination of exercise and psychotherapy was larger than for each component alone: $\mathrm{N}=5, \mathrm{~g}_{\text {combined }}=-0.845 \quad(-1.187,-$ $0.503), I^{2}=50.0 \% ; N=14, g_{\text {psychotherapy }}=-0.459(-0.683,-0.234), I^{2}=67.9 \% ; N=6$, gexercise $=-0.246(-$ $1.007,0.515), 1^{2}=81.2 \%$. The considerable heterogeneity within the exercise subgroup was partly explained by assessing separate subtypes: moderate/vigorous AE showed medium-sized benefit $\left(N=5, g=-0.571(-1.159,0.016), p=0.056, I^{2}=69.0 \%\right)$, whereas the single study with 
mixed $A E / A n E$ actually reported weight gain in the intervention group ( $N=1, g=2.699$ (1.046, 4.352). Regarding psychotherapy, the effect was very similar for all subtypes: small for psychoeducation $\left(N=5, g=-0.481(-0.823,-0.139), I^{2}=52.9 \%\right)$ and $C B T(N=6, g=-0.357(-0.653,-$ $0.061), I^{2}=47.3 \%$ ), and only slightly larger (but non-significant) for mixed psychotherapy $(N=3$, $\left.g=-0.586(-1.280 .0 .107), p=0.098, I^{2}=87.0 \%\right)$. Likewise, there were no differences in terms of mode of intervention: weight reduction was medium-sized and statistically significant both in group and individual approaches.

Splitting by diagnosis, all subgroups (SCZ, SSD, FEP) had significant medium-sized effect, except for the single study encompassing FEP + SSD patients, which had no effect whatsoever.

One study (Speyer et al., 2016) -psychotherapy in SSD outpatients- could not be pooled for meta-analysis; the authors reported no significant effect of the intervention on weight change.

\subsubsection{Weight change at the end of follow-up}

Out of the twenty-five studies examining body weight, only eight (Álvarez-Jiménez et al., 2010; Attux et al., 2013; Cheng et al., 2017; Cordes et al., 2014; Hsu et al., 2016; Littrell et al., 2003; McKibbin et al., 2010; Rotatori et al., 1980) explored follow-up persistence of the effect. Out of them, seven were suitable for meta-analysis, with an average follow-up of 23.85 weeks after the end of the intervention - totalling $\mathbf{3 8 . 8 6}$ weeks since the beginning of the study. As usual, the considered effect size measured weight change since the beginning of the intervention.

The intervention group had a comparative reduction of $-2.753 \mathrm{~kg}(-4.208,-1.298) \mathrm{p}<0.001$. This entailed follow-up persistence of a moderate effect size in favour of intervention: $g=-0.559$ (95\% $\mathrm{Cl}-0.807,-0.311) \mathrm{p}<0.001$, with $95 \%$ prediction intervals $(-1.13,0.01)$. There was no significant between-study heterogeneity: $Q^{2}=8.49(d f=6, p=0.204), \tau^{2}=0.0326, I^{2}=29.30 \%$. See figure $3 b$.

Subgroup analysis indicated no differences in effect size in terms of patient setting. Splitting by type of intervention (only exercise and psychotherapy were extant), the average effect size was non-significant for exercise studies $\left(\mathrm{N}=2, \mathrm{~g}_{\text {exercise }}=-0.232(-0.672,0.209), \mathrm{I}^{2}=0.0 \%\right)$ and moderate for psychotherapy studies ( $\left.N=5, g_{\text {psychotherapy }}=-0.654(-0.913,-0.395), I^{2}=17.7 \%\right)$. Considering specific subtypes of exercise and psychotherapy, statistical significance was observed for psychoeducation $(\mathrm{N}=1)$ and mixed psychotherapy $(\mathrm{N}=3)$, but not for moderate/vigorous $A E(N=$ 2 ) or $\mathrm{CBT}(\mathrm{N}=1)$. No difference was encountered in terms of mode of intervention (individual versus group approaches). In subgroup analysis by diagnosis, SSD-studies had the largest average effect size compared to FEP and SCZ.

One study using psychotherapy in SCZ outpatients (Rotatori et al., 1980) reported persistent weight loss in the intervention group after follow-up compared to controls, but gave no measure of effect size or statistical significance.

\subsubsection{Waist circumference}

\subsubsection{Change in waist circumference at the end of intervention}

Thirteen studies (Attux et al., 2013; Cordes et al., 2014; Holt et al., 2019; Iglesias-García et al., 2010; Lovell et al., 2014; Marzolini et al., 2009; McKibbin et al., 2006; Methapatara and 
Srisurapanont, 2011; T. W. Scheewe et al., 2013; Speyer et al., 2016; Sugawara et al., 2018; Wu et al., 2007, 2008) explored change WC as an outcome measure; all were suitable for metaanalysis.

Concerning unstandardized mean difference, there was a reduction in WC of $-1.453 \mathrm{~cm}(-2.514$, -0.392) $p=0.007$. In terms of standardized Hedges' $g$, the effect was small in size, but statistically significant: $g=-0.226,95 \% \mathrm{Cl}(-0.398,-0.054) \mathrm{p}=0.010,95 \%$ P.I $(0.750,0.300)$. There was moderate between-study heterogeneity: $Q^{2}=28.05(d f=12, p=0.005), \tau^{2}=0.0493, I^{2}=57.2 \%$. See figure $4 a$.

In subgroup analysis by setting, the inpatient subgroup had a moderate effect size in favour of intervention ( $\mathrm{N}=3$, ginpatient $\left.=-0.686(-1.173,-0.2), I^{2}=62.8 \%\right)$. Conversely, the effect was nonsignificant in outpatients $\left(N=10, g_{\text {outpatient }}=-0.086(-0.203,0.030), \mathrm{I}^{2}=8.8 \%\right)$, even though the outpatient subgroup had more studies and was largely homogeneous.

Subgroup analysis also suggested that type of intervention was a key variable in explaining out the overall between-study heterogeneity, since the three subgroups defined by this criterion (exercise, psychotherapy and combined therapy) were internally homogeneous. While the effect size was close to zero for exercise alone and for psychotherapy alone, the effect of the combined intervention was moderate: $\mathrm{N}=3, \mathrm{~g}_{\text {combined }}=-0.768 \quad(-1.082,-0.454), \quad I^{2}=6.5 \%$. Importantly, two of the three combined-intervention studies were based on inpatients, suggesting that both factors may have played a role. Considering specific subtypes of exercise and psychotherapy, all exercise-based studies had mixed AE/AnE programmes (which also reported no benefit for $\mathrm{BMI}$ and body weight). Small-sized benefit was observed for psychoeducation, but not for CBT or mixed psychotherapy. There were no differences of note by mode of intervention (group versus individual). In terms of diagnosis, SCZ subgroup ( $N=3$ ) had the largest effect size.

Funnel plot inspection showed no clear evidence of publication bias (Egger's test $p=0.095$ ).

\subsubsection{Change in waist circumference at the end of follow-up}

There were only two studies (Cordes et al., 2014; McKibbin et al., 2010) that measured change in WC at the end of a follow-up. Both had 24 weeks of follow-up, 48 in total since the beginning of the intervention.

WC reduction persisted after follow-up. In terms of unstandardized mean difference it was $W M D=-5.094 \mathrm{~cm}(-9.322,-0.866)$; standardized difference indicated small effect size (figure 4b): $g=-0.477(-0.905,-0.050) p=0.028$. There was not between-study heterogeneity: $Q^{2}=0.49(d f=$ $1, p=0.483), \tau^{2}=0.0000,1^{2}=0.0 \%$. Two issues must be considered, however: first, $p=0.028$ was (barely) no longer considered significant after FDR correction of $\alpha$-threshold to 0.025 ; second, when considering the only low-RoB study of the two, the effect for intervention was also nonsignificant.

\subsubsection{Blood glucose}




\subsubsection{Change in blood glucose at the end of intervention}

Out of the eleven studies that examined post-intervention effect on blood glucose (Attux et al., 2013; Cordes et al., 2014; Holt et al., 2018, 2019; Ikai et al., 2014; Kwon et al., 2006; McCreadie et al., 2005; McKibbin et al., 2006; T. W. Scheewe et al., 2013; Sugawara et al., 2018; Wu et al., $2007,2008)$, nine were suitable for quantitative synthesis.

Overall, there was no significant change in blood glucose compared to the control group: $\mathrm{WMD}=$ $-1.508 \mathrm{mg} / \mathrm{dL}, 95 \% \mathrm{Cl}(-6.115,3.099), \mathrm{p}=0.521$; Hedges' $\mathrm{g}=-0.212,95 \% \mathrm{Cl}(-0.550,0.126), \mathrm{p}=$ $0.219,95 \% \mathrm{PI}(-1.38,0.96)$ - see figure $5 \mathrm{a}$. However, considerable heterogeneity between studies was observed: $Q^{2}=54.38(d f=8, p<0.001), \tau^{2}=0.2161, I^{2}=85.3 \%$.

Subgroup analysis by setting encountered no effect in neither in or outpatients.

Likewise, the effect size was non-significant for all three intervention types, even though, on average, it was largest for combined intervention. Considering subtypes of exercise (mixed $\mathrm{AE} / \mathrm{AnE}$ and mind-body exercise (MBE) and psychotherapy (psychoeducation, $\mathrm{CBT}$, mixture), no intervention was beneficial in terms of blood glucose reduction; rather, $M B E(N=1)$ and $C B T(N=$ 1) were associated to higher glucose levels in the intervention group. Note, however, that no study employed standard AE. Lack of effect was also found in both group and individual approaches.

Subgroup division by diagnosis showed that the largest effect size corresponded to the single study on FEP patients, the only diagnostic subgroup with statistically significant benefit: $\mathrm{N}=1$, $g=-1.627(-2.197,-1.058))$.

Egger's test for small-study effect was significant $(p=0.029)$, but inspection of the funnel plot with enhanced contours suggested that the asymmetry was related to outlier studies rather than publication bias, and imputation by trim-and-fill method did not correct the real effect size.

Two studies, both performing psychotherapy in outpatients ((McCreadie et al., 2005)- SCZ patients- and (Kwon et al., 2006)-SSD-) did not report effect size but found no significant effect on glucose.

\subsubsection{Change in blood glucose at the end of follow-up}

There were two follow-up studies, both performing psychotherapy ((McCreadie et al., 2005)SCZ outpatients, 52 weeks of follow-up- and (Cordes et al., 2014)- SSD inpatients, 24 weeks of follow-up-). Cordes et al. was the only one giving numeric effect size - reporting significant benefit in the intervention group. McCreadie et al. only stated lack of significant effect.

\subsubsection{Total cholesterol levels}

\subsubsection{Change in total cholesterol at the end of intervention}

Six studies (Attux et al., 2013; Cordes et al., 2014; Holt et al., 2019; Ikai et al., 2014; McCreadie et al., 2005; Wu et al., 2007) examined total cholesterol after intervention; four of them were suitable for meta-analysis.

Overall, no significant effect was observed (figure $5 b): W M D=-4.273 \mathrm{mg} / \mathrm{dL}(-10.725,2.178)$, 0.194; Hedges' $\mathrm{g}=-0.097,95 \% \mathrm{Cl}(-0.249,0.054), \mathrm{p}=0.206 ; 95 \% \mathrm{PI}(-0.43,0.23)$. Moreover, there was no between-study heterogeneity: $Q^{2}=0.44(d f=3, p=0.933), \tau^{2}=0.0000, I^{2}=0.0 \%$. 
Two studies (Cordes et al., 2014; McCreadie et al., 2005) could not be pooled for quantitative synthesis but reported not significant effect.

\subsubsection{Change in total cholesterol at the end of follow-up}

As with blood glucose, two studies reported follow-up on total cholesterol (Cordes et al., 2014; McCreadie et al., 2005) but were not suitable for meta-analysis: both observed no effect after follow-up.

\subsection{6. $L D L$}

Five studies (Attux et al., 2013; Ikai et al., 2014; Kwon et al., 2006; McKibbin et al., 2006; Speyer et al., 2016) explored low density lipoproteins (LDL) change at the end of intervention (in the case of Speyer et al., actually non-high density lipoprotein (non-HDL) change, and in Kwon et al., LDL/HDL ratio). Four were suitable for meta-analysis.

Overall, no effect was observed (figure $5 c): W M D=0.010 \mathrm{mg} / \mathrm{dL}(-7.330,7.351), \mathrm{p}=0.998$; Hedges' $g=-0.002,95 \% \mathrm{Cl}(-0.168,0.164), \mathrm{p}=0.983,95 \%$ P.I. $(-0.37,0.36)$. There was no heterogeneity between studies: $Q^{2}=0.41(d f=3, p=0.939), \tau^{2}=0.0000, I^{2}=0.0 \%$.

Kwon et al. (psychotherapy in SSD outpatients) was not appropriate for meta-analysis: they mentioned no significant effect on LDL/HDL ratio (although there was some decrease in the intervention group).

No study considered follow-up LDL.

\subsection{7. $H D L$}

\subsubsection{Change in HDL at the end of intervention}

There were eight studies exploring HDL change at the end of intervention (Attux et al., 2013; Holt et al., 2019; Ikai et al., 2014; McCreadie et al., 2005; McKibbin et al., 2006; T. W. Scheewe et al., 2013; Speyer et al., 2016; Sugawara et al., 2018); seven were suitable for meta-analysis.

No overall effect was found, neither in terms of unstandardized mean difference (WMD $=1.595$ $\mathrm{mg} / \mathrm{dL}(-0.455,3.645), \mathrm{p}=0.127)$ or for standardized effect size: $\mathrm{g}=0.160,95 \% \mathrm{Cl}(-0.051,0.371)$, $p=0.138$; P.I. $(-0.47,0.79)$-see figure $5 d$. However, individual studies exhibited substantial heterogeneity: $Q^{2}=17.18(d f=6, p=0.009), \tau^{2}=0.0480, l^{2}=65.10 \%$.

All studies were based on outpatient setting. Regarding type of intervention, the effect size was non-significant for both existing subgroups, although larger for exercise $(\mathrm{N}=2, \mathrm{~g}=0.522(-0.494$, $\left.1.538), I^{2}=85.8 \%\right)$ than for psychotherapy $\left(N=5, g=0.064(-0.065,0.193), I^{2}=11.8 \%\right)$ - note that, for $\mathrm{HDL}$, beneficial effect is signalled by a positive $\mathrm{g}$, indicating $\mathrm{HDL}$ increase. Exploring intervention subtypes, two single-study subgroups had significant effect for intervention, namely mixed AE/AnE (large effect size) and psychoeducation (small effect size), while the 
others found no effect (MBE, CBT and mixed psychotherapy). Additionally, no effect was found for subgroups based on mode of intervention or diagnosis.

As with other metabolic outcomes, McCreadie et al. (McCreadie et al., 2005) did not provide numeric results but observed no effect on HDL.

\subsubsection{Change in HDL at the end of follow-up}

McCreadie et al. had a 52-week follow-up: they observed no effect on HDL but did not report effect size.

\subsubsection{Triglycerides}

\subsubsection{Change in triglycerides at the end of intervention}

Ten studies explored post-intervention effect on triglycerides (Attux et al., 2013; Cordes et al., 2014; Holt et al., 2019; Ikai et al., 2014; Kwon et al., 2006; McKibbin et al., 2006; T. W. Scheewe et al., 2013; Speyer et al., 2016; Sugawara et al., 2018; Wu et al., 2007): seven were appropriate for meta-analysis. Overall, they reported a decrease of $-25.212 \mathrm{mg} / \mathrm{dL}(-37.145,-13.279), \mathrm{p}<$ 0.001 ; this corresponded to a small but significant effect size in favour of intervention: $g=-0.273$, $95 \% \mathrm{Cl}(-0.400,-0.147), \mathrm{p}<0.001,95 \% \mathrm{PI}(-0.440,-0.110)$, with no between-study heterogeneity: $\mathrm{Q}^{2}=3.93(\mathrm{df}=6, \mathrm{p}=0.687), \tau^{2}=0.000, \mathrm{I}^{2}=0.0 \%$ (figure $5 e$ ).

The largest individual effect size corresponded to the single inpatient study with combined intervention on SCZ patients; thus, inpatient subgroup, combined intervention and SCZ subgroup had moderate effect size ( $\mathrm{N}=1$ all), while the other subgroups based on setting (outpatients, $\mathrm{N}=6$ ), intervention type (exercise with $\mathrm{N}=2$, psychotherapy with $\mathrm{N}=4$ ) and diagnosis (SSD with $\mathrm{N}=5, \mathrm{FEP}+\mathrm{SSD}$ with $\mathrm{N}=1$ ) had small effect size. There were no noticeable differences in terms of subtype of exercise/psychotherapy or mode of intervention (group vs. individual). There were no studies specifically on FEP patients; nevertheless, removal of the single study including FEP + SSD participants yielded comparable results.

Funnel plot inspection and Egger test for small-study effect $(p=0.036)$ were suggestive of publication bias. However, after imputation of missing studies by non-parametric trim and fill method, the adjusted effect size remained significant: $N=9$ ( 7 observed +2 imputed), $g=-0.234$ $(-0.354,-0.115)$.

Three studies performing psychotherapy in SSD inpatients (Cordes et al., 2014) or outpatients (Kwon et al., 2006; Speyer et al., 2016) could not be pooled for meta-analysis; they reported no significant effect.

\subsubsection{Change in triglycerides at the end of follow-up}

Cordes et al. (psychotherapy in SSD inpatients) was the only study with follow-up data, reporting no significant effect. 


\subsubsection{Systolic blood pressure (SBP)}

\subsubsection{Change in SBP at the end of intervention}

Eleven studies examined post-intervention change in SBP (Armstrong et al., 2016; Attux et al., 2013; Brar et al., 2005; Cordes et al., 2014; Holt et al., 2019; Kwon et al., 2006; Marzolini et al., 2009; McKibbin et al., 2006; T. W. Scheewe et al., 2013; Speyer et al., 2016; Sugawara et al., 2018); ten were appropriate for meta-analysis.

Overall, no significant effect was observed: $W M D=-1.296 \mathrm{mmHg}(-3.53,0.938), p=0.256$; Hedges' $g=-0.126,95 \% \mathrm{Cl}(-0.33,0.079), p=0.229,95 \% \mathrm{PI}(-0.730,0.480)$-see figure $5 f$. There was substantial between-study heterogeneity: $Q^{2}=24.45, d f=9, p=0.004, \tau^{2}=0.0585, I^{2}=$ $63.20 \%$.

All studies were performed on outpatients. All subgroups based on intervention type or subtype, mode or diagnosis yielded no significant effect. However, meta-regression (applied to low RoB studies, $\mathrm{N}=7$ ) by type and total time of intervention suggested a trend toward greater SBP reduction in exercise-based studies $(B=-0.416$, Monte-Carlo permutation test: $p=0.089)$.

Egger test for small-study effects was near-significant $(p=0.094)$, but trim-and-fill method applied no correction to the observed effect size.

One study using psychotherapy in SSD inpatients (Cordes et al., 2014) could not be pooled for quantitative synthesis but reported no change in SBP.

\subsubsection{Change in SBP at the end of follow-up}

Cordes et al. reported no effect of intervention after 24 weeks of follow-up (48 weeks since the beginning of the study)(Cordes et al., 2014).

\subsubsection{Diastolic blood pressure (DBP)}

\subsubsection{Change in DBP at the end of intervention}

There were eight studies on DBP change at the end of intervention (Armstrong et al., 2016; Attux et al., 2013; Cordes et al., 2014; Holt et al., 2019; Kwon et al., 2006; McKibbin et al., 2006; T. W. Scheewe et al., 2013; Sugawara et al., 2018); seven were suitable for meta-analysis. They reported no overall effect: $W M D=-0.258 \mathrm{mmHg}(-2.104,1.589), p=0.785$; Hedges' $g=-0.028$, $95 \% \mathrm{Cl}(-0.189,0.134), \mathrm{p}=0.735,95 \% \mathrm{PI}(-0.370,0.320)$-see figure $5 \mathrm{~g}$. Furthermore, there was no significant between-study heterogeneity: $Q^{2}=7.95 \mathrm{df}=6 p=0.242, \tau^{2}=0.0113, I^{2}=24.60 \%$.

All studies were conducted in outpatients; subgroup analysis revealed no differences in terms of intervention or patients' diagnosis.

As for SBP, Cordes et al. could not be quantitatively pooled but reported no significant change in DBP. 


\subsubsection{Change in DBP at the end of follow-up}

Cordes et al. reported no effect after follow-up.

\subsubsection{Cardiovascular risk}

McCreadie et al. -psychotherapy based on diet advice in SCZ outpatients- examined potential effect on cardiovascular risk by the Framingham assessment, at the end of intervention and after a 52-week follow-up. They gave no numeric results, but reported no effect at any time point (McCreadie et al., 2005).

Speyer et al. -psychotherapy in SSD outpatients- also assessed 10-year cardiovascular risk by the Copenhagen risk score. They reported no benefit of intervention (Speyer et al., 2016).

\subsection{Psychopathology and clinical outcomes}

\subsubsection{Positive and Negative Syndrome Scale (PANSS) total score}

\subsubsection{Change in PANSS total score at the end of intervention}

Twenty-two studies (Beebe et al., 2005; Brar et al., 2005; Caponnetto et al., 2019; Cordes et al., 2014; Curcic et al., 2017; Ikai et al., 2013, 2014, 2017; Isuru et al., 2015; Kaltsatou et al., 2015; Kang et al., 2016; Kwon et al., 2006; Lin et al., 2015; McCreadie et al., 2005; Pajonk et al., 2010; T. W. Scheewe et al., 2013; Silva et al., 2015; Svatkova et al., 2015; Varambally et al., 2012; Visceglia and Lewis, 2011; Wang et al., 2018; Wu et al., 2008) considered change in PANSS total score at the end of the intervention. Out of these, eighteen were suitable for meta-analysis. Three studies (Lin et al., 2015; Silva et al., 2015; Varambally et al., 2012) provided two intervention groups, each consisting on a different subtype of exercise intervention; therefore, twenty-one intervention/control group comparisons entered the meta-analysis.

As shown in figure $6 a$, the overall improvement was of moderate size: $\mathrm{g}=-0.596,95 \% \mathrm{Cl}(-0.828$, -0.364), $\mathrm{p}<0.001 ; 95 \% \mathrm{PI}(-1.570,0.380)$. Substantial between-study heterogeneity was present: $Q^{2}=79.94, d f=20, p<0.001, \tau^{2}=0.2027, I^{2}=75.00 \%$. In terms of unstandardized mean difference (one study, (Pajonk et al., 2010), could not be pooled in this manner), the effect entailed a decrease in -7.351 points, $95 \% \mathrm{Cl}(-10.980,-3.722), \mathrm{p}<0.001$.

Subgroup analysis by patient setting showed that the effect was of similar, medium size for both in and outpatients.

In subgroup analysis by intervention type, only exercise $(\mathrm{N}=17)$ showed significant benefit; for psychotherapy and combined intervention ( $\mathrm{N}=2$ each), the effect was small-sized and nonsignificant. However, heterogeneity was substantial within all three subgroups. Considering specific subtypes of exercise, light $A E(N=1)$ showed no significant benefit, whereas moderate/vigorous $A E(N=5)$ and $M B E(N=6)$ had medium-sized effect. Mixed exercise (mixture of $\mathrm{AE}$ and another modality: either $\mathrm{MBE}$ or anaerobic exercise; $\mathrm{N}=4$ ) and anaerobic exercise ( $A n E, N=1$ ) had both large effect size. As for subtypes of psychotherapy, $C B T(N=1)$ did not prove beneficial, while mixed psychotherapies (psychoeducation $+\mathrm{CBT} ; \mathrm{N}=1$ ) had large effect size. 
As for mode of intervention, all studies employed group therapies.

Considering subgroups by diagnosis, the largest benefit was observed in SCZ ( $N=13, g=-0.793$ ($\left.1.137,-0.449), I^{2}=79.3 \%\right)$, followed by FEP $\left(N=3, g=-0.793(-1.137,-0.449), I^{2}=76.1 \%, p=0.052\right)$ and SSD (where it was non-significant: $\left.\mathrm{N}=5, \mathrm{~g}=-0.271(-0.608,0.066), \mathrm{I}^{2}=0.115\right)$.

Egger's test indicated significant small-study effect $(p=0.038)$. However, most studies responsible for the funnel plot asymmetry were outside the pseudo $99 \%$ confidence intervals and thus unlikely to result from publication bias. Consequently, trim-and-fill method did not apply any correction to the effect size.

Four studies were not suitable for quantitative synthesis: Caponnetto et al. -yoga therapy in SCZ inpatients- (Caponnetto et al., 2019); Beebe et al., 2005, -exercise in SCZ outpatients- (Beebe et al., 2005), McCreadie et al. -psychotherapy in SCZ outpatients- (McCreadie et al., 2005); and Kwon et al. -psychotherapy in SSD outpatients- (Kwon et al., 2006). None of them reported a significant effect of the intervention compared to the control group; however, Caponnetto et al. informed of an improvement in both intervention and control groups (but only significant in the former), and Beebe et al. observed a non-significant improvement in the intervention group.

\subsubsection{Change in PANSS total score at the end of follow-up}

Four studies (Cordes et al., 2014; Lin et al., 2015; McCreadie et al., 2005; Wang et al., 2018), considered total PANSS after an average of 49 weeks since the beginning and 30 since the end of intervention. Three studies (comprising four intervention cohorts) were suitable for pooling of effect sizes.

Improvement of moderate size remained in the intervention group after end of follow-up: WMD $=-10.069$ points $(-16.005,-4.133)$; Hedges' $g=-0.625(-0.906,-0.344), p<0.001,95 \%$ P.I. ($1.420,0.170)$. See figure $6 b$.

All subgroups based on setting and type of intervention had significant effect - the single study in both the inpatient and psychotherapy subgroups had the largest effect size. All subgroups based on diagnosis were also significant; the FEP subgroup had smaller effect size.

One study, based on psychotherapy in SCZ outpatients (McCreadie et al., 2005) did not provide numeric effect size, but reported lack of effect after 52 weeks of follow-up.

\subsubsection{Positive and negative symptoms}

\subsubsection{Change in positive or negative symptoms at the end of intervention}

Twenty-four studies examined post-intervention change in positive and negative symptoms, separately: twenty employed the PANSS (positive/negative symptoms) scale (Curcic et al., 2017; Fisher et al., 2020; Ho et al., 2016; Ikai et al., 2017, 2014, 2013; Isuru et al., 2015; Kaltsatou et al., 2015; Kang et al., 2016; Lin et al., 2015; Loh et al., 2016; McCreadie et al., 2005; OertelKnöchel et al., 2014; Paikkatt et al., 2015; Sailer et al., 2015; Silva et al., 2015; Svatkova et al., 2015; Varambally et al., 2012; Visceglia and Lewis, 2011; Wang et al., 2018) and four the SAPS/SANS (Acil et al., 2008; Bhatia et al., 2017; Jayaram et al., 2013; Speyer et al., 2016) . Out of these, twenty-one were suitable for meta-analysis. Four studies (Ho et al., 2016; Lin et al., 
2015; Silva et al., 2015; Varambally et al., 2012) had two active exercise based-interventions (each being a different subtype of exercise); thus, there were in total twenty-five intervention groups suitable for meta-analysis. All twenty-five examined for both positive and negative dimensions separately. Another study also had two active interventions ( $A E$ and relaxation therapy), but the latter did not fulfil our inclusion criteria (Oertel-Knöchel et al., 2014).

The benefit of intervention was statistically significant for both dimensions. For positive symptoms (figure 6c), it was small-sized: $\mathrm{g}=-0.42695 \% \mathrm{Cl}(-0.633,-0.219), \mathrm{p}<0.001 ; 95 \% \mathrm{PI}(-$ $1.35,0.49)$, with substantial between-study heterogeneity: $Q^{2}=98.31 \mathrm{df}=24 \mathrm{p}<0.001, \tau^{2}=$ $0.1869, \mathrm{I}^{2}=75.60 \%$. For negative symptoms (figure $6 \mathrm{e}$ ) it was of moderate size: $\mathrm{g}=-0.539$, $(-$ $0.778,-0.300) p<0.001,95 \%$ P.I. $(-1.65,0.58)$, again in presence of substantial heterogeneity: $Q^{2}=132.33 \mathrm{df}=24 p<0.001, \tau^{2}=0.2753, I^{2}=81.90 \%$.

\subsection{Subgroup analyses}

Subgroup analysis by employed scale (PANSS or SAPS/SANS) produced similar, small effect sizes in all cases, except for PANSS in regards to negative symptoms, where the effect was mediumsized but with considerable heterogeneity. Division by setting indicated that the effect size was small for inpatients and medium for outpatients; this was true for positive (inpatients: $\mathrm{N}=8$, $g_{\text {inpatient positive }}=-0.271(-0.548,0.005), p=0.054, I^{2}=53.7 \%$; outpatients: $N=17$, goutpatient positive $=-$ $\left.0.521(-0.801,-0.241), I^{2}=80.6 \%\right)$ and for negative symptoms (inpatients: $\mathrm{N}=8, \mathrm{~g}_{\text {inpatient negative }}=-$ $0.228(-0.434,-0.022), I^{2}=22.0 \%$; outpatients: $N=17$, goutpatient negative $=-0.228(-0.434,-0.022), I^{2}=$ $86.7 \%)$.

Most interventions were based on exercise ( $\mathrm{N}=22)$, and had medium-sized effect, while the single psychotherapy study found none. Only the effect of combined interventions $(N=2)$ differed between psychopathological dimensions, being non-significant for positive and medium-sized for negative symptoms. Positive symptoms: $g_{\text {exercise }}=-0.507(-0.747,-0.268), I^{2}=$ $75.0 \%$; g gsychotherapy $=-0.184(-0.416,0.048) ; g_{\text {combined }}=0.152(-0.091,0.395), I^{2}=0 \%$. Negative symptoms: $g_{\text {exercise }}=-0.602(-0.882,-0.323), I^{2}=81.8 ; g_{\text {psychotherapy }}=0.083(-0.149,0.315) ; g_{\text {combined }}=$ $-0.537(-0.784,-0.290), I^{2}=0.0 \%$.

Considering specific subtypes of exercise, the effect was non-significant for light $A E(N=2)$; conversely, moderate/vigorous $A E(N=7)$ had small effect on positive symptoms and mediumsized on negative symptoms. $\mathrm{MBE}(\mathrm{N}=9)$ had small effect size. The effect size of mixed exercise (combining two modalities, $\mathrm{N}=3$ ) was large, although only significant for negative symptoms. AnE also had large effect size $(N=1)$. As for subtypes of psychotherapy, the single study was based on CBT, and was also the only study applying an individual (rather than group) approach.

According to patients' diagnosis, the effect on positive symptoms was similar for SCZ ( $N=19, \mathrm{~g}=$ $\left.-0.499(-0.782,-0.216), I^{2}=80.9 \%\right)$ and FEP $\left(N=3, g=-0.524(-0.818,-0.231), I^{2}=0 \%\right)$, but nonsignificant for SSD $\left(\mathrm{N}=3, \mathrm{~g}=-0.182(-0.382,0.019), \mathrm{I}^{2}=0 \%\right)$.

Results were very similar on negative symptoms, although FEP studies observed larger effects. SCZ: $N=19, \mathrm{~g}=-0.538(-0.826,-0.251), \mathrm{I}^{2}=81.6 \%$; FEP: $\mathrm{N}=3, \mathrm{~g}=-0.996(-1.353,-0.639), \mathrm{I}^{2}=20.8 \%$; SSD: $N=3, g=-0.174(-0.582,0.234) p=0.075, l^{2}=0 \%$. Considering only non-FEP studies, or studies with explicit mention to over 5 years of disease, results were comparable to the entire sample, although significance was lost for inpatients, MBE and mixed exercise subgroups. 


\subsection{Publication bias}

For both positive and negative symptoms, the funnel plot was asymmetrical, with significant Egger test for small-study effects; however, trim-and-fill method did not apply correction to the observed effect size.

Three studies could not be pooled for meta-analysis. One (McCreadie et al., 2005) psychotherapy on SCZ outpatients- assessed PANSS positive and negative subscales; another (Sailer et al., 2015) -exercise on SCZ inpatients- measured only PANSS negative symptoms; whereas another (Bhatia et al., 2017) -exercise on SCZ outpatients- measured SAPS and SANS scores. All reported lack of comparative benefit in the intervention group.

\subsubsection{Change in positive or negative symptoms at the end of follow-up}

Four studies investigated follow-up change in positive or negative symptoms for between 12 60 weeks since the end of intervention (Bhatia et al., 2017; Ho et al., 2016; Lin et al., 2015; Wang et al., 2018). Out of them, three studies and five intervention groups were suitable for quantitative synthesis, all of them using PANSS.

Overall, no significant effect persisted on positive symptoms $(g=-0.193(-0.557,0.172) p=0.300$, $95 \%$ P.I. $(-1.44,1.06))$ - see figure $6 \mathrm{~d}$. Negative symptoms exhibited small effect $(g=-0.425$ ($0.748,-0.102) p=0.036,95 \%$ P.I. $(-1.48,0.63))$, although the observed $p$ value was slightly above the FDR-corrected $\alpha$-threshold for statistical significance $(0.027)$-see figure $6 f$. There was substantial between-study heterogeneity for both positive $\left(Q^{2}=13.46 \mathrm{df}=4 p=0.009, \tau^{2}=0.1203\right.$, $\left.I^{2}=70.30 \%\right)$ and negative symptoms $\left(Q^{2}=10.36 \mathrm{df}=4 p=0.035, \tau^{2}=0.0822, I^{2}=61.40 \%\right)$.

Subgroup analysis observed significant benefit in outpatient studies, moderate/vigorous AE (but not in MBE) and FEP (but not SCZ) patients. This was true for both symptom dimensions; however, regarding negative symptoms, the mean effect size in SCZ patients was larger than for FEP and approached statistical significance.

One study had low RoB: (Lin et al., 2015), which employed two exercise interventions (moderate/vigorous $\mathrm{AE}$ and $\mathrm{MBE}$ ) on FEP outpatients, reporting significant effect for both positive and negative symptoms.

Only for negative symptoms, small-study effect was observed (Egger's test $p=0.002$ ) in relation to an apparent outlier; trim-and-fill method did not apply correction to the observed effect size.

One study (Bhatia et al., 2017) did not report numeric effect sizes: they studied two interventions on SCZ outpatients (AE and MBE) and observed no effect on SAPS/SANS after 24 weeks of follow-up.

\subsubsection{PANSS general symptoms}

\subsubsection{Change in PANSS general score at the end of intervention}

Thirteen studies explored post-intervention effect on PANSS general symptoms (Curcic et al., 2017; Fisher et al., 2020; Ikai et al., 2017, 2014, 2013; Isuru et al., 2015; Kaltsatou et al., 2015; Kang et al., 2016; Lin et al., 2015; Loh et al., 2016; Silva et al., 2015; Visceglia and Lewis, 2011; Wang et al., 2018), totalling fifteen intervention cohorts. 
An overall reduction of $\mathrm{WMD}=-3.261$ points was observed (95\% $\mathrm{Cl}(-5.432,-1.089), \mathrm{p}=0.003)$, corresponding to medium-sized effect in standardized terms: $g=-0.527(-0.905,-0.148), p=$ $0.006,95 \% \mathrm{PI}(-2.05,1.00)$. There was considerable between-study heterogeneity: $Q^{2}=109.62$ $(d f=14, p<0.001), \tau^{2}=0.4589, I^{2}=87.20 \%$. See figure $6 g$.

Subgroup analysis indicated similar effect size for in and outpatient setting. All interventions were exercised-based but one, which combined exercise and psychotherapy and observed no effect. Regarding subtype of exercise, light $A E$, moderate/vigorous $A E$, $A n E$ and $M B E$ proved effective (MBE had $p=0.063)$, while mixed exercises $(n=2)$ did not. All were group interventions.

In terms of diagnosis, both SCZ and FEP studies observed medium-sized effect, while SSD was non-significant.

Contour-enhanced funnel plot indicated a pattern of asymmetry that appeared related to outliers rather than publication bias (Egger test: $p=0.056$ ); indeed, trim-and-fill method applied no correction to the observed effect.

\subsubsection{Change in PANSS general symptoms at the end of follow-up}

Two studies (comprising three intervention groups) explored effect on PANSS general symptoms score after 12 and 60 weeks of follow-up (Lin et al., 2015; Wang et al., 2018). The effect persisted in similar amount as immediately after intervention: unstandardized -3.987 points (-6.566, 1.407); standardized $g=-0.457(-0.731,-0.183) p<0.001,95 \%$ PI $(-2.24,1.32)$. No significant between-study heterogeneity was extant: $Q^{2}=1.12 \mathrm{df}=2 p=0.571, \tau^{2}=0.000, I^{2}=0.0 \%$. See figure $6 h$.

\subsubsection{Brief Psychiatric Rating Scale (BPRS)}

Two studies (Holt et al., 2019; Ryu et al., 2020) explored post-intervention change in BPRS. No significant effect was observed: $g=-0.237(-1.074,0.601) p=0.580$. In terms of unstandardized mean difference: $W M D=-3.246(-13.292,6.799) p=0.526$. There was considerable betweenstudy heterogeneity: $Q^{2}=7.77, d f=1, p=0.005, \tau^{2}=0.3215, I^{2}=87.10 \%$. See figure $7 a$.

No follow-up data was available.

\subsubsection{Depression}

\subsubsection{Change in depression score at the end of intervention}

Nine studies examined post-intervention effect on depression, using different scales: Beck Depression Inventory, BDI (Ryu et al., 2020; Sailer et al., 2015); Montgomery-Åsberg Depression Rating Scale, MADRS (T. W. Scheewe et al., 2013); Calgary Depression Scale, CDS (Lin et al., 2015; Lovell et al., 2014; Silva et al., 2015); 9-item Patient Health Questionnaire, PHQ-9 (Holt et al., 2019); a depression factor obtained from PANSS (Ho et al., 2016) and the depression subscale from Mental Health Inventory, MHI (Marzolini et al., 2009). Eight of these studies were suitable for quantitative synthesis, totalling eleven intervention groups. 
As depicted in figure $7 \mathrm{~b}$, the effect in favour of intervention was medium-sized: $g=-0.535$ ($0.873,-0.196) p=0.002$, with $95 \% \operatorname{PI}(-1.72,0.65)$. There was substantial between-study heterogeneity: $Q^{2}=60.23 \mathrm{df}=10 p<0.001, \tau^{2}=0.2464, I^{2}=83.4 \%$.

Depression measured by BDI, MADRS and CDS was associated to (near) significant effect, while PANSS, MHI and PHQ-9 were not. Significant improvement was found in outpatients (but not inpatients), exercise (but not psychotherapy) and group (but not individual) approaches. Concerning specific intervention subtypes, moderate/vigorous $A E$, AnE and mixed exercises had significant effect, while MBE did not. As for psychotherapy, neither psychoeducation nor CBT were effective.

Regarding diagnosis, significant improvement was observed for both SCZ and FEP, but not SSD $(p=0.097)$ or FEP+SSD.

Importantly, funnel plot inspection and Egger's test revealed the existence of publication bias; after correction by trim-and-fill imputation method, the overall effect was no longer significant: $\mathrm{N}=14$ (11 observed +3 imputed), $g=-0.276(-0.612,0.061) \mathrm{p}=0.108$.

The study of Sailer and colleagues (Sailer et al., 2015) could not be pooled quantitatively, but informed of an overall improvement in the entire sample, without significant differences between intervention and control groups.

\subsubsection{Change in depression score at the end of follow-up}

Two studies (Ho et al., 2016; Lin et al., 2015) examined depressive symptoms after 12 and 60 weeks of follow-up, respectively. In total, four intervention groups were available: the only individual cohort that showed persisting benefit was the moderate/vigorous AE group in Ho et al. after 12 weeks of follow-up. Overall, no effect remained, as shown in figure 7c: $g=-0.054$ ($0.314,0.206) \mathrm{p}=0.684$, with $95 \% \mathrm{PI}(-0.95,0.84)$. There was no significant heterogeneity: $\mathrm{Q}^{2}=$ $4.69 \mathrm{df}=3 \mathrm{p}=0.196, \tau^{2}=0.0254, \mathrm{I}^{2}=36.1 \%$.

\subsubsection{Anxiety}

Three studies explored post-intervention change in anxiety. Two of them (Oertel-Knöchel et al., 2014; Ryu et al., 2020) examined post-intervention change in the State-Trait Anxiety Inventory -STAI-. Oertel-Knöchel and colleagues used only the state-anxiety subscale, whereas the group of Ryu reported both -state and trait- subscales separately. A third study (Marzolini et al., 2009) reported the anxiety subscale of the MHI. State anxiety and the MHI subscale were pooled for meta-analysis.

As seen in figure $7 d$, there was no overall effect on state anxiety: $g=-0.257(-1.043,0.530) p=$ 0.522 . There was substantial between-study heterogeneity: $Q^{2}=9.98, d f=2, p=0.007, \tau^{2}=$ $0.3750, I^{2}=80.0 \%$.

Regarding trait anxiety -TRAI-, Ryu et al. (Ryu et al., 2020) -exercise in SCZ outpatients- observed significant improvement in the intervention group compared to controls.

No study explored follow-up values. 


\subsubsection{Perceived stress scale (PSS)}

\subsubsection{Change in PSS at the end of intervention}

There were two studies (Caponnetto et al., 2019; Ho et al., 2016) and three intervention groups examining change on perceived stress scale (PSS), as one of the studies (Ho et al., 2016) used two exercise groups (AE and tai-chi). Both had uncertain/high RoB.

No overall effect was detected (figure 7e): $g=0.094(-0.229,0.416), p=0.569,95 \% \operatorname{PI}(-2.79,2.98)$ - unstandardized $W M D=0.731(-1.548,3.01) p=0.530$. There was not significant between-study heterogeneity: $Q^{2}=2.85 d f=2 p=0.241, \tau^{2}=0.0246, I^{2}=29.80 \%$.

\subsubsection{Change in PSS at the end of follow-up}

A single study had follow-up results on PSS, performing two active interventions (AE and tai-chi) on SCZ inpatients, followed them for 12 weeks after end of intervention. No significant effect was observed in relation to any of the two interventions (Ho et al., 2016).

\subsubsection{Rosenberg self-esteem scale (RSES)}

Two studies (Attux et al., 2013; Ryu et al., 2020) ascertained the RSES, finding no postintervention effect (figure 7f): $g=0.215(-0.377,0.807), p=0.477$, with unstandardized WMD= $1.072(-1.569,3.714) p=0.426$. There was considerable between-study heterogeneity: $Q^{2}=5.26$ $d f=1 p=0.022, \tau^{2}=0.2037, I^{2}=81.0 \%$. No follow-up results were extant.

\subsubsection{Clinical Global Impressions (CGI)}

Two studies, both applying psychotherapy in SSD patients, but differing in setting: inpatients (Cordes et al., 2014) and outpatients (Brar et al., 2005), examined clinical condition by the Clinical Global Impressions (CGI) scale. Only Cordes et al. provided numeric data on CGI-S (state), reporting no effect at the end of intervention or after 24-week follow-up (Cordes et al., 2014). Brar et al. mentioned no effect on CGI-S, but greater proportion of patients classified as '(very) much improved' in the intervention group (Brar et al., 2005).

\subsubsection{Antipsychotic drug adherence}

Two outpatient studies (Lin et al., 2015; Lovell et al., 2014) ascertained adherence to antipsychotic therapy by the BARS (Brief Adherence Rating Scale) and CRS (Compliance Rating Scale), respectively; totalling three intervention groups. No post-intervention effect was found for any of the three, nor overall: $g=-0.008(-0.253,0.238) p=0.951,95 \%$ P.I. $(-1.60,1.58) ; Q^{2}=$ 
$0.93, d f=2 p=0.629, \tau^{2}=0.000, I^{2}=0.0 \%$ - see figure 7g. Furthermore, Lin et al. (Lin et al., 2015) confirmed such lack of effect after 60-week follow-up.

\subsubsection{Various psychopathological and clinical outcomes}

Several outcomes were investigated each by a single study and thus, could not be pooled in meta-analysis. The results are briefly described in the supplementary material.

\subsubsection{Cognitive outcomes}

\subsubsection{Digit span test}

Two studies (Ho et al., 2016; Lin et al., 2015), comprising four intervention groups, evaluated explored short-term memory and attention by the WAIS (Wechsler Adult Intelligence Scale) forward and backward digit span tests. Significant effect was observed at the end of intervention, of small size for digit span forward $(g=0.309(0.062,0.556) p=0.014,95 \%$ P.I. ($0.49,1.10) ; Q^{2}=4.22, d f=3 p=0.239, \tau^{2}=0.0183 I^{2}=28.8 \%$ - figure 8a) and medium size for the backward modality $\left(g=0.621(0.288,0.953) p<0.001,95 \%\right.$ P.I. $(-0.72,1.96) ; Q^{2}=7.31, d f=3 p=$ $0.063, \tau^{2}=0.0676 I^{2}=59.0 \%$ - figure $\left.8 c\right)$. However, the effect did not persist at the end of followup neither for forward $\left(g=-0.132(-0.600,0.337) p=0.528,95 \%\right.$ P.I. $(-2.24,1.98), Q^{2}=14.96, d f=$ $3 p=0.002, \tau^{2}=0.1826 I^{2}=80.0 \%$-figure $\left.8 b\right)$ or backward test $(g=-0.201(-0.574,0.172) p=0.291$, 95\% P.I. $(-1.79,1.38), Q^{2}=9.54, d f=3, p=0.023, \tau^{2}=68.5 \% I^{2}=0.0993$-figure $\left.8 d\right)$.

\subsubsection{Other}

Different cognitive outcomes were evaluated each by a single study. The results are briefly described in the supplementary material.

\subsection{Functionality and QoL outcomes}

\subsubsection{Self-reported physical activity}

\subsubsection{Change in self-reported physical activity at the end of intervention}

Seven studies employed questionnaires for self-reported physical activity: three (Attux et al., 2013; Lovell et al., 2014; Sailer et al., 2015) used the International Physical Activity Questionnaire (IPAQ); one (McKibbin et al., 2006) the Yale Physical Activity Survey (YPAS); one (Ryu et al., 2020) the Korean version of the physical activity scale for the elderly (K-PASE); 
another (McCreadie et al., 2005) the Scottish Physical Activity Questionnaire; and yet another (Speyer et al., 2016) the Physical Activity Scale. Four of these were appropriate for metaanalysis.

No overall effect was found (figure 9a): $g=0.036(-0.219,0.29) p=0.782 ; 95 \%$ PI $(-0.83,0.90)$. There was no significant between-study heterogeneity: $Q^{2}=4.60(d f=3, p=0.204), \tau^{2}=0.0235$, $\mathrm{I}^{2}=34.8 \%$.

All four studies that entered meta-analysis were performed in outpatients. No effect was found for any subgroup based on characteristics of the intervention or the patients.

Three studies did not report data suitable for effect size pooling, but observed no effect of intervention: two of them using psychotherapy in outpatients, one with SCZ (McCreadie et al., 2005) and other with SSD (Speyer et al., 2016), and Sailer et al., using exercise in FEP+SSD inpatients (Sailer et al., 2015).

\subsubsection{Change in self-reported physical activity at the end of follow-up}

McCreadie et al. did not provide numeric data, but noted that lack of effect remained the same after 52-week follow-up (McCreadie et al., 2005).

\subsubsection{Walking}

\subsubsection{Change in walking amount at the end of intervention}

Two studies examined amount of physical activity in terms of walking: one by recording daily steps with a pedometer (Ryu et al., 2020), and another by monitoring walking time by trained personnel (Beebe et al., 2011). In the latter study the authors did not exactly measure change between pre- and post-intervention but difference between first and last month of intervention.

A small increase in favour of intervention was observed: $g=0.368(0.023,0.713) p=0.037$ (figure $9 b)$. Note, however, that the observed $p$ value was no longer significant after FDR-correction of the $\alpha$-threshold (to 0.028). There was no significant between-study heterogeneity: $Q^{2}=1.09$ ( $d f=$ $1, p=0.296), \tau^{2}=0.0058, l^{2}=8.50 \%$. The single low-RoB study found significant benefit by itself.

\subsubsection{Change in walking amount at the end of follow-up}

Beebe et al., 2013 (Beebe et al., 2013) presented follow-up data for the intervention reported in Beebe et al., 2011 (Beebe et al., 2011). Eighty-eight weeks after the end of intervention, participants wore pedometers for a week, demostrating a larger number of daily steps in the exercise group compared to controls. However, the absence of equivalent pre-intervention measurements prevented conversion into suitable effect sizes.

\subsubsection{Accelerometry}

Two studies employed accelerometry to measure amount of physical activity: daily time of moderate or vigorous physical activity (MVPA) (Holt et al., 2019); minutes of activity (McKibbin et al., 2006) 
No significant post-intervention effect was observed (figure 9c): $g=0.105(-0.075,0.285) p=$ 0.252 (unstandardized $W M D=1.979 \min (-1.493,5.451) p=0.264)$, with no between-study heterogeneity: $Q^{2}=0.93(d f=1, p=0.334), \tau^{2}=0.0000, l^{2}=0.0 \%$.

No follow-up data was available.

\subsubsection{Peak $\mathrm{O2}$}

Six studies (Armstrong et al., 2016; Curcic et al., 2017; Lin et al., 2015; T. W. Scheewe et al., 2013; Speyer et al., 2016; Svatkova et al., 2015) explored post-intervention effect on physical performance by measuring peak $\mathrm{VO}_{2}$ change. In total, seven intervention groups were available.

Overall, there was a small effect in favour of intervention: $g=0.356(0.080,0.632) p=0.011,95 \%$ $\mathrm{PI}=(-0.45,1.16)$ - with unstandardized $\mathrm{WMD}=1.660 \mathrm{~mL} /(\mathrm{kg} \cdot \mathrm{min})(0.488,2.832) \mathrm{p}=0.006$. Between-study heterogeneity was moderate: $Q^{2}=15.44(d f=6 p=0.017), \tau^{2}=0.0787, l^{2}=61.10 \%$ (figure 9d).

The largest effect size corresponded to the single inpatient study. Conversely, the only psychotherapy-based study (CBT) produced no effect at all. Moderate/vigorous AE ( $n=3)$ conveyed larger effect than MBE $(n=1)$ or mixed exercises $(n=2)$. Regarding diagnosis, SCZ studies $(n=2)$ observed medium-sized benefit, while FEP $(n=2)$ and SSD $(n=3)$ had small, nonsignificant effect.

The funnel plot and Egger test suggested a trend toward small-study effect $(p=0.081)$, but trimand-fill method did not correct the observed effect size.

There was no follow-up data.

\subsubsection{Heart rate (HR)}

Three studies (Cordes et al., 2014; Hsu et al., 2016; Speyer et al., 2016) explored postintervention HR; two of them (Cordes et al., 2014; Hsu et al., 2016) also had follow-up data. Only Hsu provided data in a manner suitable for calculation of between-group Hedges' g (Hsu et al., 2016). None observed significant effect.

\subsubsection{Peak power output (W peak)}

Two studies (T. W. Scheewe et al., 2013; Svatkova et al., 2015) examined effect on peak power output $\left(W_{\text {peak }}\right)$ as a measure of cardiovascular fitness. There was no overall effect (figure 9e): $g=$ $0.106(-0.294,0.507) p=0.604$-in unstandardized terms: WMD $=25.008$ Watt $(-67.928,117.945)$. Between-study heterogeneity was non-significant: $Q^{2}=0.00, d f=1 p=0.949, \tau^{2}=0.0000, I^{2}=0.0 \%$. There was no follow-up information. 


\subsubsection{Six-minute walking test (6MWT).}

Four studies explored physical capacity by 6MWT performance (Armstrong et al., 2016; Beebe et al., 2005; Kaltsatou et al., 2015; Marzolini et al., 2009); three of them were suitable for metaanalysis. All considered the effect immediately after intervention but had no follow-up results.

A large effect size in favour of intervention was encountered: $g=0.857(0.150,1.564) p=0.018$, $95 \% \mathrm{PI}(-6.37,8.09)$; unstandardized $\mathrm{WMD}=67.414 \mathrm{~m}(36.212,98.615) \mathrm{p}<0.001$. There was no significant between-study heterogeneity: $Q^{2}=4.00(d f=2, p=0.136), \tau^{2}=0.1935, l^{2}=50.00 \%$ (figure 9f).

Results from Beebe et al., 2005 (Beebe et al., 2005) -exercise in SCZ outpatients- could not be quantitatively pooled, but they reported no significant effect at any time point.-

\subsubsection{Various tests for physical performance}

Different physical performance outcomes were evaluated each by a single study. See supplementary material.

\subsubsection{Global functioning}

\subsubsection{Change in global functioning at the end of intervention}

Twelve studies explored global functioning measured by different scales: the Global Assessment of Functioning (GAF) (Bhatia et al., 2017; Ikai et al., 2017; Kaltsatou et al., 2015; Ryu et al., 2020; Speyer et al., 2016); the Personal and Social Performance scale (PSP) (Loh et al., 2016); the Social and Occupational Scale (SOFS) (Jayaram et al., 2013; Varambally et al., 2012); the Functional Assessment for Comprehensive Treatment of Schizophrenia (FACT-Sz) (Ikai et al., 2014, 2013); the World Health Organisation Disability Assessment (WHODAS) (Fisher et al., 2020) and the Mini-ICF-APP Social Functioning scale (Caponnetto et al., 2019). Ten of these studies, totalling eleven intervention groups, could be pooled by meta-analysis. Because higher scores meant better or worse functioning in different scales, the sign of the effect size was adjusted if pertinent so that a positive $g$ indicated improvement in the intervention group.

The overall benefit was small but significant (figure 10a): $g=0.347(0.158,0.536) p<0.001,95 \%$ PI $(-0.13,0.83)$. There was not significant heterogeneity: $Q^{2}=16.08(d f=10 p=0.0 .097), \tau^{2}=$ $0.0355, I^{2}=37.80 \%$.

All assessment scales produced similar effect sizes. The effect was medium-sized for inpatients and small for outpatients, but the first subgroup had considerable heterogeneity. The single psychotherapy study (CBT) was not effective; this was also the only study with individual approach. As for specific subtypes of exercise, the effect was small for both light AE and MBE, and large for moderate/vigorous AE. Regarding diagnosis, the smallest (and non-significant) effect size was in SSD patients, while SCZ and FEP showed similar benefit. 
Funnel plot asymmetry and Egger test $(p=0.011)$ pointed to possible existence of publication bias; trim-and-fill imputation method adjusted the effect size accordingly, which nonetheless remained significant: $n=15$ (11 observed +4 imputed), $g_{\text {observed }+ \text { imputed }}=0.213(0.004,0.422)$.

Effect sizes could not be computed for two studies: Bhatia et al. (Bhatia et al., 2017)-yoga and AE in SCZ outpatients- and Caponnetto et al. (Caponnetto et al., 2019)- yoga in SCZ inpatients-. Bhatia et al. informed of no effect in neither of the two cohorts (yoga and exercise), whereas Caponnetto et al. observed significantly improved functioning at the endpoint in the yoga group.

\subsubsection{Change in global functioning at the end of follow-up}

Only Bhatia et al. examined global functioning after follow-up (24 weeks); they did not report effect sizes, but no effect was found in any of the two interventions (Bhatia et al., 2017).

\subsubsection{QoL: overall score}

Eleven studies reported post-intervention effect on overall QoL (QoL), measured by different scales: the QoL Enjoyment and Satisfaction scale (Q-LES) (Kaltsatou et al., 2015), the World Health Organization QoL scale (WHOQOL) (Fisher et al., 2020; Ryu et al., 2020), the EuroQoL-5D (EQ-5D) (Caponnetto et al., 2019; Holt et al., 2019; Ikai et al., 2017, 2014, 2013; Lovell et al., 2014; Speyer et al., 2016), the 36-item Short Form Health Survey (SF36) (Silva et al., 2015) and the Manchester Short Assessment of QoL (MANSA) (Speyer et al., 2016). Out of these, nine studies (ten intervention groups) were suitable for meta-analysis. The sign of the effect size was made uniform across scales.

The effect size was small (figure 10b): $g=0.298(0.023,0.573) p=0.033,95 \%$ PI $(-0.540,1.130)$. The observed $p$ value was slightly above the FDR-corrected $\alpha$-threshold for statistical significance (0.026). There was substantial between-study heterogeneity: $Q^{2}=25.00(d f=9, p=$ $0.003), \tau^{2}=0.1112, I^{2}=64.0 \%$.

Subgroup analysis showed that all employed scales were associated to positive effect sizes (though mostly non-significant) except EQ5D, which was virtually non-existent. According to setting, the inpatient subgroup $(n=2)$ had large effect size, while it was non-significant in outpatients $(n=8)$. In terms of type of intervention, psychotherapy $(n=2)$ was ineffective; this applied to both psychoeducation and CBT (with a single study each). Regarding subtypes of exercise, the largest effect sizes corresponded to moderate/vigorous $A E(n=3)$ and $\operatorname{AnE}(n=1)$; the benefit was small and non-significant for $\operatorname{MBE}(n=3)$ and virtually non-existent for mixed exercises. Regarding mode of intervention, the single study with individual approach was not effective. In terms of diagnosis, only the SCZ subgroup had significant effect size.

Funnel plot and Egger test $(p=0.029)$ suggested some small-study effect, but trim-and-fill method did not modify the observed effect size.

Two studies: Speyer et al. (Speyer et al., 2016) - psychotherapy in SSD outpatients - and Caponnetto et al. (Caponnetto et al., 2019)- MBE in SCZ inpatients- did not provide groupspecific QoL scores at baseline and thus effect sizes could not be computed. Speyer et al. 
reported no significant post-intervention differences in MANSA or EQ5D, whereas Caponnetto et al. informed of significantly higher post-intervention EQ5D in the yoga group.

No study reported follow-up results.

\subsubsection{QoL: physical component}

Twelve studies measured physical QoL: five employed the WHOQOL scale (Acil et al., 2008; Attux et al., 2013; Kang et al., 2016; Kwon et al., 2006; Visceglia and Lewis, 2011); four, the 36-item Short Form Health Survey (SF36)(Lin et al., 2015; Loh et al., 2016; Lovell et al., 2014; Silva et al., 2015); two, the 12-item Short Form (SF12) (Battaglia et al., 2013; Oertel-Knöchel et al., 2014); and another, the QLES (Kaltsatou et al., 2015). In total, fourteen intervention groups were available. All of these considered effect at the end of intervention, with no follow-up data.

A medium-sized effect was observed in favour of intervention (figure 10c): $g=0.596(0.319$, $0.874) \mathrm{p}<0.001,95 \% \mathrm{PI}(-0.38,1.58)$; there was substantial between-study heterogeneity: $\mathrm{Q}^{2}=$ $50.05(d f=13, p<0.001), \tau^{2}=0.1822, I^{2}=74.00 \%$. The scale employed was not associated to effect size.

Considering the employed scale for QoL assessment, all subgroups had positive effect size, though it was smaller for WHOQOL. Effect was medium-sized for both in and outpatient settings. However, while exercise interventions $(n=9)$ had large-sized benefit, it was non-significant for psychotherapy $(n=3)$ and combined intervention $(n=2)$. Considering exercise subtypes, the effect was medium-sized for light $A E$ and $M B E$; and large for moderate/vigorous $A E, A n E$ and mixed exercises. Regarding subtypes of psychotherapy, the single CBT study had near-significant effect, unlike psychoeducation and mixed psychotherapies. Both group and individual approaches had positive effect (smaller and near-significant for individual).

According to patients' diagnosis, both SCZ and FEP had medium-sized effect, while for SSD it was smaller and non-significant.

Funnel plot and Egger test $(p=0.001)$ indicated possible publication bias, although the corrected effect size by trim-and-fill imputation remained significant: $n=18$ (14 observed +4 imputed), gobserved + imputed $=0.392(0.121,0.662)$.

\subsubsection{QoL: mental component}

Ten studies, comprising twelve intervention groups, explored mental QoL at the end of intervention: four employed the WHOQOL scale (Acil et al., 2008; Attux et al., 2013; Kang et al., 2016; Visceglia and Lewis, 2011); four, the SF36 (Lin et al., 2015; Loh et al., 2016; Lovell et al., 2014; Silva et al., 2015) one, the SF12 (Battaglia et al., 2013), and another, the QLES (Kaltsatou et al., 2015).

The effect size in favour of the intervention was small (figure 10d): $g=0.370(0.126,0.614) p=$ $0.003,95 \% \mathrm{PI}(-0.41,1.15)$. There was substantial between-study heterogeneity: $Q^{2}=31.49$ (df= $11, p=0.001), \tau^{2}=0.1062, I^{2}=65.1 \%$. 
Subgroups based on employed QoL scale produced similar effects (albeit for the single SF-12 study, which was larger). Likewise, in and outpatient settings had similar effect sizes, although the inpatient subgroup ( $n=3)$ was non-significant. Exercise interventions $(n=9)$ were associated to medium-sized benefit, while for psychotherapy $(n=2)$ and combined intervention $(n=1)$ it was very small and non-significant. Regarding specific subtypes of exercise, light $A E(n=1)$ and AnE $(n=1)$ were not effective; moderate/vigorous $A E(n=4)$ and mixed exercises $(n=1)$ had medium-sized effect, and MBE $(n=2)$ had large-size benefit. There was no substantial difference between group and individual interventions.

Concerning diagnosis, the largest effect size was encountered in the FEP subgroup $(n=2)$; both SCZ $(n=7)$ and SSD $(n=3)$ had small, non-significant effect. Sensitivity analysis on non-FEP studies $(n=10)$ yielded slightly smaller effect sizes, but otherwise comparable to the entire sample although the exercise subgroup barely lost significance $(p=0.064)$. When only studies with explicit reports of over 5 years since diagnosis were considered $(n=5)$, mean effect sizes remained comparable, but statistical significance was lost in most cases.

In meta-regression by setting, type and time of intervention, exercise was non-significantly associated to larger effect $(B=.8525$, permutation-generated $p=0.074)$.

Funnel plot inspection and Egger test $(p=0.046)$, provided some evidence of publication bias; nevertheless, after correction by trim-and-fill method, the effect size remained significant: $n=$ 15 (12 observed, 3 imputed), gobserved + imputed $=0.290(0.048,0.531)$.

As with other QoL components, no follow-up was available.

\subsubsection{QoL: social component}

Seven studies (eight intervention groups) considered post-intervention effect on social QoL: four employed the WHOQOL scale (Acil et al., 2008; Attux et al., 2013; Kang et al., 2016; Visceglia and Lewis, 2011); two, the SF36 (Loh et al., 2016; Silva et al., 2015); and another, the QLES (Kaltsatou et al., 2015). There was no follow-up information.

Moderate effect size was observed in favour of intervention (figure 10e): $g=0.648(0.219,1.076)$ $\mathrm{p}=0.003,95 \% \mathrm{PI}(-0.74,2.04)$. There was substantial between-study heterogeneity: $\mathrm{Q}^{2}=38.83$ $(d f=7, p<0.001), \tau^{2}=0.2753, l^{2}=82.0 \%$.

According to scale, WHOQOL subgroup had the smallest (non-significant) effect size. In and outpatient settings conveyed similar benefit. Regarding intervention type, exercise $(n=6)$ was associated to large effect size, while it was virtually non-existent for psychotherapy and combined intervention ( $n=1$ each). Light $(n=1)$ and moderate/vigorous $A E(n=2)$, as well as MBE $(n=1)$, had all medium-sized effect (though non-significant for MBE); for AnE and mixed exercises ( $n=1$ each), the effect was large. All studies were group-based. Concerning diagnosis, all studies enrolled SCZ patients except a single SSD study that showed no effect; there were no FEP populations.

Funnel plot inspection and Egger test $(p=0.018)$ were suggestive of small-study effect; however, trim-and-fill method did not apply correction to the observed effect size. 


\subsubsection{QoL: environmental component}

Four studies considered environmental QoL (Acil et al., 2008; Attux et al., 2013; Kang et al., 2016; Visceglia and Lewis, 2011) all of them employed the WHOQOL scale and only considered change at the end of intervention.

The overall effect size in favour of intervention was not statistically significant (figure 10f): $\mathrm{g}=$ $0.171(-0.014,0.355) p=0.071 ; 95 \%$ PI $(-0.24,0.58)$; FDR-corrected $\alpha$-threshold for statistical significance was 0.030 . There was no between-study heterogeneity: $Q^{2}=0.98(d f=3, p=0.806)$, $\tau^{2}=0.0000, I^{2}=0.0 \%$.

\subsubsection{Various measurements on psychosocial functioning}

Several outcomes of psychosocial functioning were examined each one in a single study and are described in the supplementary material. 


\section{Discussion}

\subsection{Summary of general findings}

This systematic review and meta-analysis examined the effectivity on multiple outcomes of nonpharmacological interventions such as physical activity or psychotherapy focused on lifestyle changes based on diet and exercise, performed on patients with non-affective psychosis. To the best of our knowledge, this is the first systematic review focused on the whole spectrum of nonaffective psychosis (including FEP), based on both, in and outpatient settings, exploring a wide range of outcomes including anthropometric measures and metabolic parameters, clinical and psychopathological symptoms, cognitive domains, cardiovascular fitness, QoL and overall functioning.

Briefly, an overall benefit at the end of intervention was observed for anthropometric measures (BMI, weigh and WC), psychotic symptoms severity, many cognitive domains and tests of physical performance, global functioning and QoL; conversely, there was no effect in relation to other metabolic parameters (except triglycerides), non-psychotic psychopathology (e.g., anxiety and depression, although for depression a medium-sized effect was observed, which became non-significant after correction for publication bias) and amount of physical activity (although an effect was observed for walking steps, which became non-significant after FDR correction). In general, benefit was greater for interventions involving exercise alone or in combination with psychotherapy, -especially moderate/vigorous aerobic exercise (AE)-, although this was not observed for all the outcomes. There might also be greater effectivity associated to group compared to individual interventions, although there were very few of the latter. The number of studies providing follow-up results was smaller, and available only for some outcomes. The benefit persisted after follow-up in anthropometric measures (although for WC, significance was lost after FDR correction) and several psychopathological domains such as PANSS total, general and negative symptoms (the latter became barely non-significant after FDR correction). Conversely, follow-up effect disappeared for triglycerides, positive psychotic symptoms, depression, digit span test and most cognitive outcomes, and global functioning. Follow-up effect in anthropometric measures seemed to be greater in relation to psychotherapy (unlike for results immediately after intervention), although the small number precluded strong conclusions.

Overall effect sizes, when significant, were usually medium-sized $(0.5-0.8)$. The largest effects were found for post-intervention BMI and six-minute walking test (6MWT). Follow-up effects were small to medium sized, when significant. There was substantial between-study heterogeneity for most outcomes.

\subsection{Post-intervention effects}

\subsubsection{Effects by type of intervention}

Interventions involving exercise alone or in combination with psychotherapy appeared to have greater effectivity than psychotherapy alone in several outcomes immediately after the end of 
intervention - though large heterogeneity between studies must be considered. In the case of anthropometric parameters and blood triglycerides, the greatest effect was observed for combined interventions. Regarding specific subtypes of interventions, moderate/vigorous AE also appeared associated to larger effect sizes in those outcomes. These results should be taken into account as it has been shown that triglyceride levels and WC are significantly associated with 10-year risk of coronary heart disease (Correll et al., 2006), being WC the strongest predictor for type-2- diabetes and cardiovascular diseases (Alberti et al., 2007). It is worth noting that other studies applying combined therapy that did not meet criteria for inclusion in our systematic review (non-RCT design or inclusion of other mental illness diagnosis or caring staff) also found significant reductions of anthropometric and metabolic parameters (Forsberg et al., 2008; Melamed et al., 2008; Poulin et al., 2007; Vreeland et al., 2003). Moreover, a systematic review of non-pharmacological interventions on weight gain among FEP patients found combined interventions seemed to be more effective than lifestyle counselling (Nyboe et al., 2019).

A potential association has been established between use of antipsychotics with high risk of metabolic syndrome -olanzapine and clozapine- (Leucht et al., 2020) and metabolic and anthropometric outcomes. It could be expected that those studies with greater prevalence of these drugs would report smaller effect of metabolic outcomes. However, we decided not to include this potential predictor in meta-regression as percent of patients taking clozapine/olanzapine was not reported in many studies (see supplementary table 1 ) and study level averages or proportions may produce spurious findings in relation to ecological fallacy. Further studies are needed to precisely explore the difference in response to cardiometabolic interventions in relation to antipsychotic treatment.

Moreover, effectivity was also found for exercise-based interventions on physical and cardiorespiratory performance, which has been associated with risk reduction for cardiovascular diseases and associated mortality. Several measurements of physical performance showed postintervention improvement, which was of large size for 6MWT; however, most studies did not find a consistent increase in spontaneous physical activity (measured by self-reported questionnaires and amount of walking and accelerometery) which casts doubts over the longterm maintenance. These findings are consistent with other exercise studies, such as a 14-week pilot program in SCZ patients without control group (Abdel-Baki et al., 2013). Evidence shows that the improvement in cardiorespiratory fitness is correlated with brain volumetric changes in several cortical areas: an increased volume of hippocampus, decreased ventricular and increased cerebral grey matter volume, thickening in the frontal, temporal and cingulate cortex of the left hemisphere (Scheewe et al., 2013).

These volumetric changes may partly explain the improvement in cognitive performance associated to exercise-based interventions. In this meta-review, we identified many studies reporting post-intervention improvements in several cognitive outcomes, although only Digit Span Test (a measure for short term memory) was suitable for meta-analysis, also finding postintervention effect in both, forward and backward modalities. In line with this, studies have proposed exercise-driven increase in brain-derived neurotrophic factor as a possible mechanism for reduction of cognitive deficits in chronic SCZ (Kim et al., 2014; Kimhy et al., 2015, 2014). Another possible mechanism might involve improvement in cardiovascular function: exercisedriven increase in peak $\mathrm{O} 2$ has been found to correlate with cognitive improvement (Angevaren et al., 2008). Another meta-analysis focusing on cognition demonstrated improved global 
cognition, working memory, social cognition and attention after aerobic exercise in SCZ patients (Firth et al., 2018). Deficits in verbal Short Term Memory and processing speed are the most sensitive domains to exercise; importantly benefit has been observed in both FEP (Andersen et al., 2013) and long-term SCZ patients (Leutwyler et al., 2014), and is predictive of overall functioning (Fett et al., 2011). In a recent meta-review, Ashdown-Franks and colleagues have suggested that exercise may be beneficial as an adjunctive therapy for mental disorders indicating improvement in global cognition as well as positive and negative symptoms of schizophrenia (Ashdown-Franks et al., 2020)

In our own assessment of the effect on psychopathology, we identified medium-sized postintervention effect over PANSS total score, negative symptoms and general symptoms, and small effect for positive symptoms. Importantly, exercise interventions were associated to larger effect size than other lifestyle interventions; this applied to most subtypes of exercise, including $A E, A n E, M B E$, etc, without consistent differences between subtypes. Conversely, no effect was found for exercise interventions on non-psychotic symptomatology, except for depression, where all subtypes of exercise but MBE had significant effect. In relation to this, three previous meta-analyses found significant benefit of small to medium effect size for $A E$ and MBE on negative symptoms (Sabe et al., 2020, 2019; Vogel et al., 2019), but in presence of substantial heterogeneity, which also applies to our data set Another meta-analysis found that MBE was more beneficial than other forms of physical exercise with regard to improvement of total PANSS score, but only two RCT were included and authors considered evidence as 'low quality' (Li et al., 2018).

Improvements in cognition, psychopathology and physical performance may drive the encountered benefits of lifestyle interventions on global functioning and QoL. As stated above, cognition in schizophrenia patients is correlated with global functioning (Fett et al., 2011). Furthermore, previous evidence collected that severity of psychopathology has a strong negative impact on QoL among older adults with SSD and is mainly mediated through two broad dimensions of psychopathology, i.e., negative and cognitive symptoms are significantly associated with poorer functional outcome (Lepage et al., 2014). Several studies have associated negative symptoms with psychosocial functioning (Kurtz, 2006; Lysaker et al., 2009; Ochoa et al., 2005; Ventura et al., 2013) and psychosocial functioning with health-related quality of life (HRQoL) (Browne et al., 1996). The present meta-analysis did found beneficial effect of exercise (but not psychotherapy) on global functioning and QoL outcomes, in accordance with a metaanalysis pointing exercise as a robust add-on treatment for improving QoL and overall functioning (Dauwan et al., 2016), and with another study comparing effects of training combined with cognitive remediation in multi-episode SCZ patients versus healthy controls demonstrating positive effect on everyday functioning in patients (Malchow et al., 2015). In our results, most subtypes of exercises were significantly associated to the majority of outcomes; moderate/vigorous AE had large effect on global functioning, overall and physical QoL, but medium size for mental and social QoL; likewise, AnE had large effect on overall QoL, physical and social QoL, but was non-significant for mental QoL. MBE had small to moderate effect size on most QoL outcomes, except for mental QoL, where it achieved large effect size. However, note that the number of studies per exercise subtype was small $(1-4)$ and there was important heterogeneity.

Contrary to exercise, the effect of psychotherapy immediately at the end of intervention was more discrete in general and often non-existent. A benefit was found for anthropometric 
measurements (BMI and body weight); indeed, for body weight, the effect of psychotherapy was larger than for exercise alone, and combined interventions showed the higher effect size for BMI, weight and WC. Concerning subtypes of psychotherapy, psychoeducation, CBT and mixture of both had all significant effect on BMI, while for weight, psychoeducation and CBT were significant, and for WC, with fewer studies, only psychoeducation was significant. Conversely, no effect of any studied psychotherapies was found on most psychopathology and clinical outcomes, as well as physical performance, global functioning and QoL; however, for most of these outcomes, psychotherapy studies were the minority.

\subsubsection{Effects by diagnosis}

One of the a priori aims of this review was to investigate whether benefits could be found at chronic stages of psychosis and not only in participants with FEP and early psychosis. In the latter, effectivity was likely to be greater due to their better clinical condition and less exposure to antipsychotic drugs. Previous studies have also observed successful results from lifestyle interventions in FEP on metabolic outcomes (Nyboe et al., 2019), psychopathology, social functioning and several cognitive domains (Firth et al., 2018). While the number of FEP studies in our meta-review is small to draw strong conclusions (Álvarez-Jiménez et al., 2010, 2006; Fisher et al., 2020; Holt et al., 2018; Lin et al., 2015; Lovell et al., 2014; Sailer et al., 2015), subgroup analyses by patient diagnosis revealed comparable effectivity in FEP patients and chronic disease for most outcomes, and sensitivity analyses on non-FEP studies produced similar results to the whole sample, even when analyses were restricted to studies that explicitly mention over five years of evolution of disease (see supplementary materials for more details). Therefore, as most studies were based on chronic stages of psychosis, benefit in these patients seemed established, albeit often in presence of substantial between-study heterogeneity.

\subsubsection{Effects by setting}

As secondary objective, this review aimed to study the influence of the setting where experimental intervention is performed. We were expecting to find a greater effect size in relation to inpatient setting, as hospitalization may facilitate personalized multidisciplinary interventions (Ward et al., 2015) and a more intensive dietary intake monitorization (Melamed et al., 2008), ensuring a greater adherence and less dropout rates (Gagliardi et al., 2016; Vancampfort et al., 2016). Contrary to our expectations, our results only revealed greater effect size in the inpatient setting for BMI, WC, triglycerides, overall functioning and overall quality of life; and similar or even larger benefit was found for activities implemented in the outpatient setting for most of the clinical and psychopathological outcome. At this point, it should be noted that only 13 of 57 studies (without counting the follow-up studies) were based in inpatients, and therefore, robust conclusions could not be drawn. Interestingly, the supposed benefits attributed to the inpatient setting in the literature (Stubbs et al., 2016) might not be the real results of interventions, rather than inpatient basal level of activity could be higher than outpatients and those living in the community (Vancampfort et al., 2017), and that the most important predictor of dropouts might be the qualification of professionals providing the physical interventions, which may tailor more individualized and supervised intervention (Stubbs et al., 2018; Vancampfort et al., 2015), and not the setting by itself. 


\subsection{Follow-up effects}

Despite the apparent superior post-intervention effect of exercise-based interventions, psychotherapy seems to be at least as effective or more in maintaining the improvement in anthropometric measures after follow-up. However, this result is supported by relatively few studies. An interpretation is that while exercise drives greater immediate effect in physical and mental domains, psychotherapy may be more effective in promoting long-term life style changes by using theoretical frameworks through behavioural change approaches that can increase adherence and maintain results highlighting the importance of autonomous motivation. In line with self-determination theory (Deci and Ryan, 2000), Vancampfort et al. postulated that people with SCZ may manage barriers to physical activity with support from three psychological constructs, namely autonomy, competence and relatedness (Vancampfort et al., 2013).

The improvement in psychiatric symptoms (PANSS total score, positive, negative and general dimensions) was maintained after follow-up, with large effect size for psychotherapy (only available for PANSS total score), and moderate/vigorous AE, while MBE had no significant effect. Follow-up effect of psychopathology was greater in outpatient setting.

\subsection{Limitations and strenghts}

This review has several limitations. First, the relatively small number of studies involving variables of interest (e.g., FEP patients, specific antipsychotic intake, specific types and modes of the intervention, etc), precluded in-depth examination of interesting questions on the effectivity of interventions or lead to lack of statistical significance. Second, funnel plots and Egger's tests for small study effect indicated the likely existence of publication bias in several outcomes, which might yield skewed results with overestimated effect size; moreover, many studies which did not provide numeric results and that could not be entered in meta-analysis explicitly mentioned lack of effect. Nevertheless, the effect size of outcomes affected by publication bias was corrected by trim-and-fill method of imputation of missing studies. Third, most outcomes showed considerable between-study heterogeneity which could not be solved by subgroup division or moderator analyses; in fact, even for those outcomes which a large overall effect size, predictive intervals indicated that some individual studies could be expected not to have an effect. Fourth, studies considering cognitive effects employed different and heterogeneous tests and cognitive batteries assessing specific domain, which prevented metanalysis of results (except for digit span test).

Conversely, this study has some strengths. The main one is the exhaustive examination of a wide range of outcomes. We aimed to assess the effectivity of any intervention based on exercise or promotion of metabolic health even if they did not primarily seek metabolic outcomes (for instance, exercise-based interventions focused exclusively on emotional wellbeing were also considered). In addition, we explored specific subtypes of exercise and psychotherapy, defined by type and intensity of intervention. While other similar systematic reviews were focused on antipsychotic intake and considered any diagnosis including non-psychotic diagnosis or affective psychosis, our review focussed exclusively on patients with non-affective psychosis, given the special needs of these vulnerable patient group and greater impairment associated to disease progression. Moreover, most articles included were of reasonable quality according to Cochrane 
criteria for the explored risk of bias in terms of random sequence generation, reporting bias, detection bias and attrition; however, there was a high number of studies with unclear or high risk of allocation concealment. In order to rule out spurious findings driven by los-quality studies, sensitivity analyses were performed repeating overall and subgroup analyses for all outcomes in the subset of low risk of bias, with similar results to the entire data set.

\subsection{Future research and general conclusions}

In conclusion, physical activity and lifestyle interventions are feasible and effective in nonaffective psychotic and FEP patients, with benefit in a broad variety of outcomes. Results show that there is a wide margin for improvement in the physical and mental condition of these patients, with apparently greater effect size in relation to interventions including physical exercise, and mainly moderate/vigorous aerobic exerciselmportantly, benefit can be achieved in all stages of the disease and not only in early episodes. More studies are needed in order to identify specific features of interventions that help to maintain this beneficial effect long after the end of intervention. It would also be desirable to apply standardized testing protocols (e.g. pre-defined scales to test for specific cognitive domains for all RCTs in the field) and structured evaluation to carry out a systematic exploration of the different outcomes (from physical to quality of life) in this type of studies. In summary, the final objective would be to tailor interventions to each participant's preferences and needs, in order to make healthy lifestyle accessible and intrinsically motivating.

\section{References}

Abdel-Baki, A., Brazzini-Poisson, V., Marois, F., Letendre, É., Karelis, A.D., 2013. Effects of aerobic interval training on metabolic complications and cardiorespiratory fitness in young adults with psychotic disorders: A pilot study. Schizophr. Res. 149, 112-115. https://doi.org/10.1016/j.schres.2013.06.040

Acil, A.A., Dogan, S., Dogan, O., 2008. The effects of physical exercises to mental state and quality of life in patients with schizophrenia. J. Psychiatr. Ment. Health Nurs. 15, 808815. https://doi.org/10.1111/j.1365-2850.2008.01317.x

Alberti, K.G.M.M., Zimmet, P., Shaw, J., 2007. International Diabetes Federation: A consensus on Type 2 diabetes prevention, in: Diabetic Medicine. Diabet Med, pp. 451-463. https://doi.org/10.1111/j.1464-5491.2007.02157.x

Allison, D.B., Casey, D.E., 2001. Antipsychotic-induced weight gain: A review of the literature. J. Clin. Psychiatry.

Álvarez-Jiménez, M., González-Blanch, C., Vázquez-Barquero, J.L., Pérez-Iglesias, R., MartínezGarcía, O., Pérez-Pardal, T., Ramírez-Bonilla, M.L., Crespo-Facorro, B., 2006. Attenuation of antipsychotic-induced weight gain with early behavioral intervention in drug-naive first-episode psychosis patients: A randomized controlled trial. J. Clin. Psychiatry 67, 1253-1260. https://doi.org/10.4088/JCP.v67n0812

Álvarez-Jiménez, M., Martínez-García, O., Pérez-Iglesias, R., Ramírez, M.L., Vázquez-Barquero, J.L., Crespo-Facorro, B., 2010. Prevention of antipsychotic-induced weight gain with early behavioural intervention in first-episode psychosis: 2-year results of a randomized controlled trial. Schizophr. Res. 116, 16-19. https://doi.org/10.1016/j.schres.2009.10.012 
Andersen, R., Fagerlund, B., Rasmussen, H., Ebdrup, B.H., Aggernaes, B., Gade, A., Oranje, B., Glenthoj, B., 2013. The influence of impaired processing speed on cognition in firstepisode antipsychotic-naïve schizophrenic patients. Eur. Psychiatry 28, 332-339. https://doi.org/10.1016/j.eurpsy.2012.06.003

Angevaren, M., Aufdemkampe, G., Verhaar, H.J.J., Aleman, A., Vanhees, L., 2008. Physical activity and enhanced fitness to improve cognitive function in older people without known cognitive impairment. Cochrane database Syst. Rev. CD005381. https://doi.org/10.1002/14651858.CD005381.pub2

Armstrong, H.F., Bartels, M.N., Paslavski, O., Cain, D., Shoval, H.A., Ballon, J.S., Khan, S., Sloan, R.P., Kimhy, D., 2016. The impact of aerobic exercise training on cardiopulmonary functioning in individuals with schizophrenia. Schizophr. Res. https://doi.org/10.1016/j.schres.2016.03.009

Ashdown-Franks, G., Firth, J., Carney, R., Carvalho, A.F., Hallgren, M., Koyanagi, A., Rosenbaum, S., Schuch, F.B., Smith, L., Solmi, M., Vancampfort, D., Stubbs, B., 2020. Exercise as Medicine for Mental and Substance Use Disorders: A Meta-review of the Benefits for Neuropsychiatric and Cognitive Outcomes. Sport. Med. https://doi.org/10.1007/s40279-019-01187-6

Attux, C., Martini, L.C., Elkis, H., Tamai, S., Freirias, A., Camargo, M. das G.M., Mateus, M.D., Mari, J. de J., Reis, A.F., Bressan, R.A., 2013. A 6-month randomized controlled trial to test the efficacy of a lifestyle intervention for weight gain management in schizophrenia. BMC Psychiatry 13, 60. https://doi.org/10.1186/1471-244X-13-60

Battaglia, G., Alesi, M., Inguglia, M., Roccella, M., Caramazza, G., Bellafiore, M., Palma, A., 2013. Soccer practice as an add-on treatment in the management of individuals with a diagnosis of schizophrenia. Neuropsychiatr. Dis. Treat. 9, 595-603. https://doi.org/10.2147/NDT.S44066

Bechdolf, A., Knost, B., Kuntermann, C., Schiller, S., Klosterkötter, J., Hambrecht, M., Pukrop, R., 2004. A randomized comparison of group cognitive-behavioural therapy and group psychoeducation in patients with schizophrenia. Acta Psychiatr. Scand. 110, 21-28. https://doi.org/10.1111/j.1600-0447.2004.00300.x

Beebe, L.H., Smith, K., Burk, R., McIntyre, K., Dessieux, O., Tavakoli, A., Tennison, C., Velligan, D., 2011. Effect of a motivational intervention on exercise behavior in persons with schizophrenia spectrum disorders. Community Ment. Health J. 47, 628-636. https://doi.org/10.1007/s10597-010-9363-8

Beebe, L.H., Smith, K.D., Roman, M.W., Burk, R.C., McIntyre, K., Dessieux, O.L., Tavakoli, A., Tennison, C., 2013. A pilot study describing physical activity in persons with schizophrenia spectrum disorders (Ssds) after an exercise program. Issues Ment. Health Nurs. 34, 214219. https://doi.org/10.3109/01612840.2012.746411

Beebe, L.H., Tian, L., Morris, N., Goodwin, A., Allen, S.S., Kuldau, J., 2005. Effects of exercise on mental and physical health parameters of persons with schizophrenia. Issues Ment. Health Nurs. 26, 661-676. https://doi.org/10.1080/01612840590959551

Bhatia, T., Mazumdar, S., Wood, J., He, F., Gur, R.E., Gur, R.C., Nimgaonkar, V.L., Deshpande, S.N., 2017. A randomised controlled trial of adjunctive yoga and adjunctive physical exercise training for cognitive dysfunction in schizophrenia. Acta Neuropsychiatr. 29, 102-114. https://doi.org/10.1017/neu.2016.42 
Bobes, J., Garcia-Portilla, M.P., Bascaran, M.T., Saiz, P.A., Bousoño, M., 2007. Quality of life in schizophrenic patients. Dialogues Clin. Neurosci.

https://doi.org/10.5294/aqui.2011.11.1.5

Bonfioli, E., Berti, L., Goss, C., Muraro, F., Burti, L., 2012. Health promotion lifestyle interventions for weight management in psychosis: A systematic review and metaanalysis of randomised controlled trials. BMC Psychiatry 12. https://doi.org/10.1186/1471-244X-12-78

Brar, J.S., Ganguli, R., Pandina, G., Turkoz, I., Berry, S., Mahmoud, R., 2005. Effects of behavioral therapy on weight loss in overweight and obese patients with schizophrenia or schizoaffective disorder. J. Clin. Psychiatry 66, 205-212. https://doi.org/10.4088/JCP.v66n0208

Brown, C., Goetz, J., Hamera, E., Gajewski, B., 2014. Treatment response to the RENEW weight loss intervention in schizophrenia: Impact of intervention setting. Schizophr. Res. 159, 421-425. https://doi.org/10.1016/j.schres.2014.09.018

Browne, S., Roe, M., Lane, A., Gervin, M., Morris, M., Kinsella, A., Larkin, C., O'Callaghan, E., 1996. Quality of life in schizophrenia: Relationship to sociodemographic factors, symptomatology and tardive dyskinesia. Acta Psychiatr. Scand. 94, 118-124. https://doi.org/10.1111/j.1600-0447.1996.tb09835.x

Caemmerer, J., Correll, C.U., Maayan, L., 2012. Acute and maintenance effects of nonpharmacologic interventions for antipsychotic associated weight gain and metabolic abnormalities: A meta-analytic comparison of randomized controlled trials. Schizophr. Res. 140, 159-168. https://doi.org/10.1016/j.schres.2012.03.017

Caponnetto, P., Auditore, R., Maglia, M., Pipitone, S., Inguscio, L., 2019. Psychological wellness, yoga and quality of life in patients affected by schizophrenia spectrum disorders: A pilot study. Ment. Illn. 11. https://doi.org/10.4081/mi.2019.8003

Cheng, S.L., Sun, H.F., Yeh, M.L., 2017. Effects of an 8-week aerobic dance program on healthrelated fitness in patients with schizophrenia. J. Nurs. Res. 25, 429-435. https://doi.org/10.1097/JNR.0000000000000200

Cochran, W.G., 1954. The Combination of Estimates from Different Experiments. Biometrics 10, 101. https://doi.org/10.2307/3001666

Cohen, J., 1998. Statistical Power Analysis for the Behavioral Sciences, Statistical Power Analysis for the Behavioral Sciences. Routledge, New York. https://doi.org/10.4324/9780203771587

Cooper, S.J., Reynolds, G.P., Barnes, T.R.E., England, E., Haddad, P.M., Heald, A., Holt, R.I.G., Lingford-Hughes, A., Osborn, D., McGowan, O., Patel, M.X., Paton, C., Reid, P., Shiers, D., Smith, J., 2016. BAP guidelines on the management of weight gain, metabolic disturbances and cardiovascular risk associated with psychosis and antipsychotic drug treatment. J. Psychopharmacol. 30, 717-748.

https://doi.org/10.1177/0269881116645254

Cordes, J., Thünker, J., Regenbrecht, G., Zielasek, J., Correll, C.U., Schmidt-Kraepelin, C., LangeAsschenfeldt, C., Agelink, M.W., Kahl, K.G., Gaebel, W., Klimke, A., Hauner, H., 2014. Can an early weight management program (WMP) prevent olanzapine (OLZ)-induced disturbances in body weight, blood glucose and lipid metabolism? Twenty-four-and 48week results from a 6-month randomized trial. World J. Biol. Psychiatry 15, 229-241. 
https://doi.org/10.3109/15622975.2011.592546

Correll, C.U., Frederickson, A.M., Kane, J.M., Manu, P., 2006. Metabolic syndrome and the risk of coronary heart disease in 367 patients treated with second-generation antipsychotic drugs. J. Clin. Psychiatry 67, 575-583. https://doi.org/10.4088/JCP.v67n0408

Curcic, D., Stojmenovic, T., Djukic-Dejanovic, S., Dikic, N., Vesic-Vukasinovic, M., Radivojevic, N., Andjelkovic, M., Borovcanin, M., Djokic, G., 2017. Positive impact of prescribed physical activity on symptoms of schizophrenia: Randomized clinical trial. Psychiatr. Danub. 29, 459-465. https://doi.org/10.24869/psyd.2017.459

Dauwan, M., Begemann, M.J.H., Heringa, S.M., Sommer, I.E., 2016. Exercise improves clinical symptoms, quality of life, global functioning, and depression in schizophrenia: A systematic review and meta-analysis. Schizophr. Bull. 42, 588-599. https://doi.org/10.1093/schbul/sbv164

De Hert, M., Correll, C.U., Bobes, J., Cetkovich-Bakmas, M., Cohen, D.A.N., Asai, I., Detraux, J., Gautam, S., Möller, H.J., Ndetei, D.M., Newcomer, J.W., Uwakwe, R., Leucht, S., 2011. Physical illness in patients with severe mental disorders. I. Prevalence, impact of medications and disparities in health care. World Psychiatry. https://doi.org/10.1002/j.2051-5545.2011.tb00014.x

Deci, E.L., Ryan, R.M., 2000. The "what" and "why" of goal pursuits: Human needs and the selfdetermination of behavior. Psychol. Inq. 11, 227-268. https://doi.org/10.1207/S15327965PLI1104_01

DerSimonian, R., Laird, N., 2015. Meta-analysis in clinical trials revisited. Contemp. Clin. Trials 45, 139-145. https://doi.org/10.1016/j.cct.2015.09.002

Faulkner, G., Soundy, A.A., Lloyd, K., 2003. Schizophrenia and weight management: A systematic review of interventions to control weight. Acta Psychiatr. Scand. https://doi.org/10.1034/j.1600-0447.2003.00218.x

Fett, A.K.J., Viechtbauer, W., Dominguez, M. de G., Penn, D.L., van Os, J., Krabbendam, L., 2011. The relationship between neurocognition and social cognition with functional outcomes in schizophrenia: A meta-analysis. Neurosci. Biobehav. Rev. https://doi.org/10.1016/j.neubiorev.2010.07.001

Firth, J., Carney, R., Elliott, R., French, P., Parker, S., Mclntyre, R., McPhee, J.S., Yung, A.R., 2018. Exercise as an intervention for first-episode psychosis: a feasibility study. Early Interv. Psychiatry 12, 307-315. https://doi.org/10.1111/eip.12329

Fisher, E., Wood, S.J., Upthegrove, R., Aldred, S., 2020. Designing a feasible exercise intervention in first-episode psychosis: Exercise quality, engagement and effect. Psychiatry Res. 286. https://doi.org/10.1016/j.psychres.2020.112840

Forsberg, K.A., Björkman, T., Sandman, P.O., Sandlund, M., 2008. Physical health - A cluster randomized controlled lifestyle intervention among persons with a psychiatric disability and their staff. Nord. J. Psychiatry 62, 486-495. https://doi.org/10.1080/08039480801985179

Gage, S.H., Smith, G.D., Munafò, M.R., 2016. Schizophrenia and neighbourhood deprivation. Transl. Psychiatry. https://doi.org/10.1038/tp.2016.244

Gagliardi, A.R., Berta, W., Kothari, A., Boyko, J., Urquhart, R., 2016. Integrated knowledge translation (IKT) in health care: A scoping review. Implement. Sci. 
https://doi.org/10.1186/s13012-016-0399-1

Gaitero-Calleja, A., Santed-Germán, M., Rullas-Trincado, M., Grande de Lucas, A., 2007. Cognitive behavioral weight-loss program for individuals with psychotic mental diseases. Psicothema 19, 640-5.

Gates, J., Killackey, E., Phillips, L., Álvarez-Jiménez, M., 2015. Mental health starts with physical health: Current status and future directions of non-pharmacological interventions to improve physical health in first-episode psychosis. The Lancet Psychiatry. https://doi.org/10.1016/S2215-0366(15)00213-8

Gurusamy, J., Gandhi, S., Damodharan, D., Ganesan, V., Palaniappan, M., 2018. Exercise, diet and educational interventions for metabolic syndrome in persons with schizophrenia: A systematic review. Asian J. Psychiatr. https://doi.org/10.1016/j.ajp.2018.06.018

Henderson, D.C., Vincenzi, B., Andrea, N. V., Ulloa, M., Copeland, P.M., 2015. Pathophysiological mechanisms of increased cardiometabolic risk in people with schizophrenia and other severe mental illnesses. The Lancet Psychiatry. https://doi.org/10.1016/S2215-0366(15)00115-7

Higgins, J.P.T., Altman, D.G., Gøtzsche, P.C., Jüni, P., Moher, D., Oxman, A.D., Savović, J., Schulz, K.F., Weeks, L., Sterne, J.A.C., 2011. The Cochrane Collaboration's tool for assessing risk of bias in randomised trials. BMJ 343. https://doi.org/10.1136/bmj.d5928

Higgins, J.P.T., Thompson, S.G., 2004. Controlling the risk of spurious findings from metaregression. Stat. Med. https://doi.org/10.1002/sim.1752

Higgins, J.P.T., Thompson, S.G., Deeks, J.J., Altman, D.G., 2003. Measuring inconsistency in meta-analyses. Br. Med. J. https://doi.org/10.1136/bmj.327.7414.557

Higgins, Thomas, Chandler, Cumpston, Li, Page, Welch, 2019. Cochrane Handbook for Systematic Reviews of Interventions, 2nd Editio. ed. John Wiley \& Sons, Chichester (UK).

Ho, R.T.H., Fong, T.C.T., Wan, A.H.Y., Au-Yeung, F.S.W., Wong, C.P.K., Ng, W.Y.H., Cheung, I.K.M., Lo, P.H.Y., Ng, S.M., Chan, C.L.W., Chen, E.Y.H., 2016. A randomized controlled trial on the psychophysiological effects of physical exercise and Tai-chi in patients with chronic schizophrenia. Schizophr. Res. 171, 42-49. https://doi.org/10.1016/j.schres.2016.01.038

Holt, R.I.G., Gossage-Worrall, R., Hind, D., Bradburn, M.J., McCrone, P., Morris, T., Edwardson, C., Barnard, K., Carey, M.E., Davies, M.J., Dickens, C.M., Doherty, Y., Etherington, A., French, P., Gaughran, F., Greenwood, K.E., Kalidindi, S., Khunti, K., Laugharne, R., Pendlebury, J., Rathod, S., Saxon, D., Shiers, D., Siddiqi, N., Swaby, E.A., Waller, G., Wright, S., 2019. Structured lifestyle education for people with schizophrenia, schizoaffective disorder and first-episode psychosis (STEPWISE): Randomised controlled trial. Br. J. Psychiatry 214, 63-73. https://doi.org/10.1192/bjp.2018.167

Holt, R.I.G., Hind, D., Gossage-Worrall, R., Bradburn, M.J., Saxon, D., McCrone, P., Morris, T.A., Etherington, A., Shiers, D., Barnard, K., Swaby, L., Edwardson, C., Carey, M.E., Davies, M.J., Dickens, C.M., Doherty, Y., French, P., Greenwood, K.E., Kalidindi, S., Khunti, K., Laugharne, R., Pendlebury, J., Rathod, S., Siddiqi, N., Wright, S., Waller, G., Gaughran, F., 2018. Structured lifestyle education to support weight loss for people with schizophrenia, schizoaffective disorder and first episode psychosis: The STEPWISE RCT. Health Technol. Assess. (Rockv). 22, 1-160. https://doi.org/10.3310/hta22650

Hsu, C.C., Liang, C.S., Tai, Y.M., Cheng, S.L., 2016. Incongruent changes in heart rate variability 
and body weight after discontinuing aerobic exercise in patients with schizophrenia. Int. J. Psychophysiol. 109, 132-137. https://doi.org/10.1016/j.ijpsycho.2016.08.011

Iglesias-García, C., Toimil-Iglesias, A., Alonso-Villa, M.J., 2010. Pilot study of the efficacy of an educational programme to reduce weight, on overweight and obese patients with chronic stable schizophrenia. J. Psychiatr. Ment. Health Nurs. 17, 849-851. https://doi.org/10.1111/j.1365-2850.2010.01590.x

Ikai, S., Suzuki, T., Uchida, H., Saruta, J., Tsukinoki, K., Fujii, Y., Mimura, M., 2014. Effects of weekly one-hour hatha yoga therapy on resilience and stress levels in patients with schizophrenia-spectrum disorders: An eight-week randomized controlled trial. J. Altern. Complement. Med. 20, 823-830. https://doi.org/10.1089/acm.2014.0205

Ikai, S., Uchida, H., Mizuno, Y., Tani, H., Nagaoka, M., Tsunoda, K., Mimura, M., Suzuki, T., 2017. Effects of chair yoga therapy on physical fitness in patients with psychiatric disorders: A 12-week single-blind randomized controlled trial. J. Psychiatr. Res. 94, 194201. https://doi.org/10.1016/j.jpsychires.2017.07.015

Ikai, S., Uchida, H., Suzuki, T., Tsunoda, K., Mimura, M., Fujii, Y., 2013. Effects of yoga therapy on postural stability in patients with schizophrenia-spectrum disorders: A single-blind randomized controlled trial. J. Psychiatr. Res. 47, 1744-1750. https://doi.org/10.1016/j.jpsychires.2013.07.017

Isuru, L., Dahanayake, D., de Alwis, A., Weerasinghe, A., Hewage, S., Ranasinghe, C., 2015. Impact of dance, drama, yoga and music therapy workshops on symptom reduction in patients with Schizophrenia: A randomized controlled study | Request PDF. South Asian J. Psychiatry 3:2, 1-7.

Jayaram, N., Varambally, S., Behere, R. V., Venkatasubramanian, G., Arasappa, R., Christopher, R., Gangadhar, B.N., 2013. Effect of yoga therapy on plasma oxytocin and facial emotion recognition deficits in patients of schizophrenia. Indian J. Psychiatry 55. https://doi.org/10.4103/0019-5545.116318

Jean-Baptiste, M., Tek, C., Liskov, E., Chakunta, U.R., Nicholls, S., Hassan, A.Q., Brownell, K.D., Wexler, B.E., 2007. A pilot study of a weight management program with food provision in schizophrenia. Schizophr. Res. 96, 198-205. https://doi.org/10.1016/j.schres.2007.05.022

Kaltsatou, A., Kouidi, E., Fountoulakis, K., Sipka, C., Theochari, V., Kandylis, D., Deligiannis, A., 2015. Effects of exercise training with traditional dancing on functional capacity and quality of life in patients with schizophrenia: A randomized controlled study. Clin. Rehabil. 29, 882-891. https://doi.org/10.1177/0269215514564085

Kang, R., Wu, Y., Li, Z., Jiang, J., Gao, Q., Yu, Y., Gao, K., Yan, Y., He, Y., 2016. Effect of community-based social skills training and tai-chi exercise on outcomes in patients with chronic schizophrenia: A randomized, one-year study. Psychopathology 49, 345-355. https://doi.org/10.1159/000448195

Kim, H. jae, Song, B. kil, So, B., Lee, O., Song, W., Kim, Y., 2014. Increase of circulating BDNF levels and its relation to improvement of physical fitness following 12 weeks of combined exercise in chronic patients with schizophrenia: A pilot study. Psychiatry Res. 220, 792796. https://doi.org/10.1016/j.psychres.2014.09.020

Kimhy, D., Vakhrusheva, J., Bartels, M.N., Armstrong, H.F., Ballon, J.S., Khan, S., Chang, R.W., Hansen, M.C., Ayanruoh, L., Lister, A., Castrén, E., Smith, E.E., Sloan, R.P., 2015. The impact of aerobic exercise on brain-derived neurotrophic factor and neurocognition in 
individuals with schizophrenia: A single-blind, randomized clinical trial. Schizophr. Bull. 41, 859-868. https://doi.org/10.1093/schbul/sbv022

Kimhy, D., Vakhrusheva, J., Bartels, M.N., Armstrong, H.F., Ballon, J.S., Khan, S., Chang, R.W., Hansen, M.C., Ayanruoh, L., Smith, E.E., Sloan, R.P., 2014. Aerobic fitness and body mass index in individuals with schizophrenia: Implications for neurocognition and daily functioning. Psychiatry Res. 220, 784-791. https://doi.org/10.1016/j.psychres.2014.08.052

Kurtz, M.M., 2006. Symptoms versus neurocognitive skills as correlates of everyday functioning in severe mental illness. Expert Rev. Neurother. https://doi.org/10.1586/14737175.6.1.47

Kwon, J.S., Choi, J.S., Bahk, W.M., Kim, C.Y., Kim, C.H., Shin, Y.C., Park, B.J., Oh, C.G., 2006. Weight management program for treatment-emergent weight gain in olanzapine-treated patients with schizophrenia or schizoaffective disorder: A 12-week randomized controlled clinical trial. J. Clin. Psychiatry 67, 547-553. https://doi.org/10.4088/JCP.v67n0405

Laws, K.R., Darlington, N., Kondel, T.K., McKenna, P.J., Jauhar, S., 2018. Cognitive Behavioural Therapy for schizophrenia - outcomes for functioning, distress and quality of life: A metaanalysis. BMC Psychol. 6. https://doi.org/10.1186/s40359-018-0243-2

Lepage, M., Bodnar, M., Bowie, C.R., 2014. Neurocognition: Clinical and functional outcomes in schizophrenia. Can. J. Psychiatry. https://doi.org/10.1177/070674371405900103

Leucht, S., Cipriani, A., Spineli, L., Mavridis, D., Örey, D., Richter, F., Samara, M., Barbui, C., Engel, R.R., Geddes, J.R., Kissling, W., Stapf, M.P., Lässig, B., Salanti, G., Davis, J.M., 2013. Comparative efficacy and tolerability of 15 antipsychotic drugs in schizophrenia: A multiple-treatments meta-analysis. Lancet 382, 951-962. https://doi.org/10.1016/S01406736(13)60733-3

Leucht, S., Crippa, A., Siafis, S., Patel, M.X., Orsini, N., Davis, J.M., 2020. Dose-response metaanalysis of antipsychotic drugs for acute schizophrenia. Am. J. Psychiatry 177, 342-353. https://doi.org/10.1176/appi.ajp.2019.19010034

Leutwyler, H., Hubbard, E.M., Jeste, D. V., Miller, B., Vinogradov, S., 2014. Associations of Schizophrenia Symptoms and Neurocognition With Physical Activity in Older Adults With Schizophrenia. Biol. Res. Nurs. 16, 23-30. https://doi.org/10.1177/1099800413500845

Li, J., Shen, J., Wu, G., Tan, Y., Sun, Y., Keller, E., Jiang, Y., Wu, J., 2018. Mindful exercise versus non-mindful exercise for schizophrenia: A systematic review and meta-analysis of randomized controlled trials. Complement. Ther. Clin. Pract.

https://doi.org/10.1016/j.ctcp.2018.04.003

Lin, J., Chan, S.K., Lee, E.H., Chang, W.C., Tse, M., Su, W.W., Sham, P., Hui, C.L., Joe, G., Chan, C.L., Khong, P.L., So, K.F., Honer, W.G., Chen, E.Y., 2015. Aerobic exercise and yoga improve neurocognitive function in women with early psychosis. npj Schizophr. 1, 15047. https://doi.org/10.1038/npjschz.2015.47

Littrell, K.H., Hilligoss, N.M., Kirshner, C.D., Petty, R.G., Johnson, C.G., 2003. The effects of an educational intervention on antipsychotic-induced weight gain. J. Nurs. Scholarsh. 35, 237-241. https://doi.org/10.1111/j.1547-5069.2003.00237.x

Loh, S.Y. i., Abdullah, A., Abu Bakar, A.K. adi., Thambu, M., Nik Jaafar, N.R. uzyane., 2016. Structured Walking and Chronic Institutionalized Schizophrenia Inmates: A pilot RCT Study on Quality of Life. Glob. J. Health Sci. 8, 238-248. 
https://doi.org/10.5539/gjhs.v8n1p238

Lovell, K., Wearden, A., Bradshaw, T., Tomenson, B., Pedley, R., Davies, L.M., Husain, N., Woodham, A., Escott, D., Swarbrick, C.M., Femi-Ajao, O., Warburton, J., Marshall, M., 2014. An exploratory randomized controlled study of a healthy living intervention in early intervention services for psychosis: The intervention to encourage activity, improve diet, and reduce weight gain (INTERACT) study. J. Clin. Psychiatry 75, 498-505. https://doi.org/10.4088/JCP.13m08503

Lysaker, P.H., Vohs, J.L., Tsai, J., 2009. Negative symptoms and concordant impairments in attention in schizophrenia: Associations with social functioning, hope, self-esteem and internalized stigma. Schizophr. Res. 110, 165-172. https://doi.org/10.1016/j.schres.2009.01.015

Malchow, B., Keller, K., Hasan, A., Dörfler, S., Schneider-Axmann, T., Hillmer-Vogel, U., Honer, W.G., Schulze, T.G., Niklas, A., Wobrock, T., Schmitt, A., Falkai, P., 2015. Effects of Endurance Training Combined with Cognitive Remediation on Everyday Functioning, Symptoms, and Cognition in Multiepisode Schizophrenia Patients. Schizophr. Bull. 41, 847-858. https://doi.org/10.1093/schbul/sbv020

Marzolini, S., Jensen, B., Melville, P., 2009. Feasibility and effects of a group-based resistance and aerobic exercise program for individuals with severe schizophrenia: $A$ multidisciplinary approach. Ment. Health Phys. Act. 2, 29-36.

https://doi.org/10.1016/j.mhpa.2008.11.001

McCreadie, R.G., Kelly, C., Connolly, M., Williams, S., Baxter, G., Lean, M., Paterson, J.R., 2005. Dietary improvement in people with schizophrenia: Randomised controlled trial. Br. J. Psychiatry 187, 346-351. https://doi.org/10.1192/bjp.187.4.346

McKibbin, C.L., Golshan, S., Griver, K., Kitchen, K., Wykes, T.L., 2010. A healthy lifestyle intervention for middle-aged and older schizophrenia patients with diabetes mellitus: $A$ 6-month follow-up analysis. Schizophr. Res. 121, 203-206. https://doi.org/10.1016/j.schres.2009.09.039

McKibbin, C.L., Patterson, T.L., Norman, G., Patrick, K., Jin, H., Roesch, S., Mudaliar, S., Barrio, C., O'Hanlon, K., Griver, K., Sirkin, A., Jeste, D. V., 2006. A lifestyle intervention for older schizophrenia patients with diabetes mellitus: A randomized controlled trial. Schizophr. Res. 86, 36-44. https://doi.org/10.1016/j.schres.2006.05.010

Melamed, Y., Stein-Reisner, O., Gelkopf, M., Levi, G., Sivan, T., llievici, G., Rosenberg, R., Weizman, A., Bleich, A., 2008. Multi-modal weight control intervention for people with persistent mental disorders. Psychiatr. Rehabil. J. 31, 194-200. https://doi.org/10.2975/31.3.2008.194.200

Methapatara, W., Srisurapanont, M., 2011. Pedometer walking plus motivational interviewing program for Thai schizophrenic patients with obesity or overweight: A 12-week, randomized, controlled trial. Psychiatry Clin. Neurosci. 65, 374-380. https://doi.org/10.1111/j.1440-1819.2011.02225.x

Mitchell, A.J., Vancampfort, D., Sweers, K., Van Winkel, R., Yu, W., De Hert, M., 2013. Prevalence of metabolic syndrome and metabolic abnormalities in schizophrenia and related disorders-a systematic review and meta-analysis. Schizophr. Bull. https://doi.org/10.1093/schbul/sbr148

Moher, D., Liberati, A., Tetzlaff, J., Altman, D.G., 2009. Preferred reporting items for systematic 
reviews and meta-analyses: the PRISMA statement. J. Clin. Epidemiol. 62, 1006-1012. https://doi.org/10.1016/j.jclinepi.2009.06.005

Mucheru, D., Hanlon, M.C., McEvoy, M., Thakkinstian, A., MacDonald-Wicks, L., 2019. Comparative efficacy of lifestyle intervention strategies targeting weight outcomes in people with psychosis: A systematic review and network meta-analysis. JBI Database Syst. Rev. Implement. Reports. https://doi.org/10.11124/JBISRIR-2017-003943

Newcomer, J.W., Hennekens, C.H., 2007. Severe mental illness and risk of cardiovascular disease. J. Am. Med. Assoc. https://doi.org/10.1001/jama.298.15.1794

Nyboe, L., Lemcke, S., Møller, A.V., Stubbs, B., 2019. Non-pharmacological interventions for preventing weight gain in patients with first episode schizophrenia or bipolar disorder: A systematic review. Psychiatry Res. https://doi.org/10.1016/j.psychres.2019.112556

Ochoa, S., Haro, J.M., Usall, J., Autonell, J., Vicens, E., Asensio, F., 2005. Needs and its relation to symptom dimensions in a sample of outpatients with schizophrenia. Schizophr. Res. 75, 129-134. https://doi.org/10.1016/j.schres.2004.06.002

Oertel-Knöchel, V., Mehler, P., Thiel, C., Steinbrecher, K., Malchow, B., Tesky, V., Ademmer, K., Prvulovic, D., Banzer, W., Zopf, Y., Schmitt, A., Hänsel, F., 2014. Effects of aerobic exercise on cognitive performance and individual psychopathology in depressive and schizophrenia patients. Eur. Arch. Psychiatry Clin. Neurosci. 264, 589-604. https://doi.org/10.1007/s00406-014-0485-9

Ohi, K., Kataoka, Y., Shimada, T., Kuwata, A., Okubo, H., Kimura, K., Yasuyama, T., Uehara, T., Kawasaki, Y., 2019. Meta-analysis of physical activity and effects of social function and quality of life on the physical activity in patients with schizophrenia. Eur. Arch. Psychiatry Clin. Neurosci. 269, 517-527. https://doi.org/10.1007/s00406-018-0903-5

Paikkatt, B., Singh, A.R., Singh, P.K., Jahan, M., Ranjan, J.K., 2015. Efficacy of Yoga therapy for the management of psychopathology of patients having chronic schizophrenia. Indian J. Psychiatry 57, 355-360. https://doi.org/10.4103/0019-5545.171837

Pajonk, F.G., Wobrock, T., Gruber, O., Scherk, H., Berner, D., Kaizl, I., Kierer, A., Müller, S., Oest, M., Meyer, T., Backens, M., Schneider-Axmann, T., Thornton, A.E., Honer, W.G., Falkai, P., 2010. Hippocampal plasticity in response to exercise in schizophrenia. Arch. Gen. Psychiatry 67, 133-143. https://doi.org/10.1001/archgenpsychiatry.2009.193

Peters, J.L., Sutton, A.J., Jones, D.R., Abrams, K.R., Rushton, L., 2008. Contour-enhanced metaanalysis funnel plots help distinguish publication bias from other causes of asymmetry. J. Clin. Epidemiol. 61, 991-996. https://doi.org/10.1016/j.jclinepi.2007.11.010

Poulin, M.J., Chaput, J.P., Simard, V., Vincent, P., Bernier, J., Gauthier, Y., Lanctôt, G., Saindon, J., Vincent, A., Gagnon, S., Tremblay, A., 2007. Management of antipsychotic-incluced weight gain: Prospective naturalistic study of the effectiveness of a supervised exercise programme. Aust. N. Z. J. Psychiatry 41, 980-989.

https://doi.org/10.1080/00048670701689428

Ratliff, J.C., Palmese, L.B., Reutenauer, E.L., Liskov, E., Grilo, C.M., Tek, C., 2012. The effect of dietary and physical activity pattern on metabolic profile in individuals with schizophrenia: A cross-sectional study. Compr. Psychiatry 53, 1028-1033. https://doi.org/10.1016/j.comppsych.2012.02.003

Rotatori, A.F., Fox, R., Wicks, A., 1980. Weight loss with psychiatric residents in a behavioral self control program. Psychol. Rep. 46, 483-486. 
https://doi.org/10.2466/pr0.1980.46.2.483

Ryu, J., Jung, J.H., Kim, J., Kim, C.H., Lee, H.B., Kim, D.H., Lee, S.K., Shin, J.H., Roh, D., 2020.

Outdoor cycling improves clinical symptoms, cognition and objectively measured physical activity in patients with schizophrenia: A randomized controlled trial. J. Psychiatr. Res.

120, 144-153. https://doi.org/10.1016/j.jpsychires.2019.10.015

Sabe, M., Kaiser, S., Sentissi, O., 2020. Physical exercise for negative symptoms of schizophrenia: Systematic review of randomized controlled trials and meta-analysis. Gen. Hosp. Psychiatry. https://doi.org/10.1016/j.genhosppsych.2019.11.002

Sabe, M., Sentissi, O., Kaiser, S., 2019. Meditation-based mind-body therapies for negative symptoms of schizophrenia: Systematic review of randomized controlled trials and metaanalysis. Schizophr. Res. https://doi.org/10.1016/j.schres.2019.07.030

Sailer, P., Wieber, F., Pröpster, K., Stoewer, S., Nischk, D., Volk, F., Odenwald, M., 2015. A brief intervention to improve exercising in patients with schizophrenia: A controlled pilot study with mental contrasting and implementation intentions (MCII). BMC Psychiatry 15, 211. https://doi.org/10.1186/s12888-015-0513-y

Sawilowsky, S., 2009. New Effect Size Rules of Thumb. Theor. Behav. Found. Educ. Fac. Publ.

Scheewe, Haren, V., Sarkisyan, G., Schnack, H.G., Brouwer, R.M., de Glint, M., Hulshoff Pol, H.E., Backx, F.J.G., Kahn, R.S., Cahn, W., 2013. Exercise therapy, cardiorespiratory fitness and their effect on brain volumes: A randomised controlled trial in patients with schizophrenia and healthy controls. Eur. Neuropsychopharmacol. 23, 675-685. https://doi.org/10.1016/j.euroneuro.2012.08.008

Scheewe, T.W., Backx, F.J.G., Takken, T., Jörg, F., van Strater, A.C.P., Kroes, A.G., Kahn, R.S., Cahn, W., 2013. Exercise therapy improves mental and physical health in schizophrenia: $A$ randomised controlled trial. Acta Psychiatr. Scand. 127, 464-473.

https://doi.org/10.1111/acps.12029

Scocco, P., Longo, R., Caon, F., 2006. Weight change in treatment with olanzapine and a psychoeducational approach. Eat. Behav. 7, 115-124.

https://doi.org/10.1016/j.eatbeh.2005.08.003

Silva, B.A., Cassilhas, R.C., Attux, C., Cordeiro, Q., Gadelha, A.L., Telles, B.A., Bressan, R.A., Ferreira, F.N., Rodstein, P.H., Daltio, C.S., Tufik, S., de Mello, M.T., 2015. A 20-week program of resistance or concurrent exercise improves symptoms of schizophrenia: Results of a blind, randomized controlled trial. Rev. Bras. Psiquiatr. 37, 271-279. https://doi.org/10.1590/1516-4446-2014-1595

Speyer, H., Christian Brix Nørgaard, H., Birk, M., Karlsen, M., Storch Jakobsen, A., Pedersen, K., Hjorthøj, C., Pisinger, C., Gluud, C., Mors, O., Krogh, J., Nordentoft, M., 2016. The CHANGE trial: No superiority of lifestyle coaching plus care coordination plus treatment as usual compared to treatment as usual alone in reducing risk of cardiovascular disease in adults with schizophrenia spectrum disorders and abdominal obesity. World Psychiatry 15, 155-165. https://doi.org/10.1002/wps.20318

Stanton, R., Happell, B., 2014. A systematic review of the aerobic exercise program variables for people with schizophrenia. Curr. Sports Med. Rep. https://doi.org/10.1249/JSR.0000000000000069

Sterne, J.A., Egger, M., Moher, D., 2008. Addressing Reporting Biases, in: Cochrane Handbook for Systematic Reviews of Interventions: Cochrane Book Series. John Wiley and Sons, pp. 
Stubbs, B., Firth, J., Berry, A., Schuch, F.B., Rosenbaum, S., Gaughran, F., Veronesse, N., Williams, J., Craig, T., Yung, A.R., Vancampfort, D., 2016. How much physical activity do people with schizophrenia engage in? A systematic review, comparative meta-analysis and meta-regression. Schizophr. Res. https://doi.org/10.1016/j.schres.2016.05.017

Stubbs, B., Vancampfort, D., Hallgren, M., Firth, J., Veronese, N., Solmi, M., Brand, S., Cordes, J., Malchow, B., Gerber, M., Schmitt, A., Correll, C.U., De Hert, M., Gaughran, F., Schneider, F., Kinnafick, F., Falkai, P., Möller, H.J., Kahl, K.G., 2018. EPA guidance on physical activity as a treatment for severe mental illness: a meta-review of the evidence and Position Statement from the European Psychiatric Association (EPA), supported by the International Organization of Physical Therapists in Mental Health (IOPTMH). Eur. Psychiatry 54, 124-144. https://doi.org/10.1016/j.eurpsy.2018.07.004

Sugawara, N., Sagae, T., Yasui-Furukori, N., Yamazaki, M., Shimoda, K., Mori, T., Sugai, T., Matsuda, H., Suzuki, Y., Ozeki, Y., Okamoto, K., Someya, T., 2018. Effects of nutritional education on weight change and metabolic abnormalities among patients with schizophrenia in Japan: A randomized controlled trial. J. Psychiatr. Res. 97, 77-83. https://doi.org/10.1016/j.jpsychires.2017.12.002

Svatkova, A., Mandl, R.C.W., Scheewe, T.W., Cahn, W., Kahn, R.S., Hulshoff Pol, H.E., 2015. Physical Exercise Keeps the Brain Connected: Biking Increases White Matter Integrity in Patients with Schizophrenia and Healthy Controls. Schizophr. Bull. 41, 869-878. https://doi.org/10.1093/schbul/sbv033

Tosh, G., Clifton, A., Bachner, M., 2011. General physical health advice for people with serious mental illness, in: Cochrane Database of Systematic Reviews. John Wiley \& Sons, Ltd. https://doi.org/10.1002/14651858.cd008567.pub2

Tumiel, E., Wichniak, A., Jarema, M., Lew-Starowicz, M., 2019. Nonpharmacological Interventions for the Treatment of Cardiometabolic Risk Factors in People With Schizophrenia-A Systematic Review. Front. Psychiatry 10. https://doi.org/10.3389/fpsyt.2019.00566

Vancampfort, D., Correll, C.U., Scheewe, T.W., Probst, M., De Herdt, A., Knapen, J., De Hert, M., 2013. Progressive muscle relaxation in persons with schizophrenia: A systematic review of randomized controlled trials. Clin. Rehabil. https://doi.org/10.1177/0269215512455531

Vancampfort, D., Firth, J., Schuch, F.B., Rosenbaum, S., Mugisha, J., Hallgren, M., Probst, M., Ward, P.B., Gaughran, F., De Hert, M., Carvalho, A.F., Stubbs, B., 2017. Sedentary behavior and physical activity levels in people with schizophrenia, bipolar disorder and major depressive disorder: a global systematic review and meta-analysis. World Psychiatry 16, 308-315. https://doi.org/10.1002/wps.20458

Vancampfort, D., Rosenbaum, S., Probst, M., Soundy, A., Mitchell, A.J., De Hert, M., Stubbs, B., 2015. Promotion of cardiorespiratory fitness in schizophrenia: a clinical overview and meta-analysis. Acta Psychiatr. Scand. 132, 131-143. https://doi.org/10.1111/acps.12407

Vancampfort, D., Rosenbaum, S., Schuch, F.B., Ward, P.B., Probst, M., Stubbs, B., 2016. Prevalence and predictors of treatment dropout from physical activity interventions in schizophrenia: A meta-analysis. Gen. Hosp. Psychiatry 39, 15-23. https://doi.org/10.1016/j.genhosppsych.2015.11.008 
Vancampfort, Firth, Correll, Solmi, M., Siskind, D., De Hert, M., Carney, R., Koyanagi, A., Carvalho, A.F., Gaughran, F., Stubbs, B., 2019. The impact of pharmacological and nonpharmacological interventions to improve physical health outcomes in people with schizophrenia: a meta-review of meta-analyses of randomized controlled trials. World Psychiatry 18, 53-66. https://doi.org/10.1002/wps.20614

Vancampfort, Probst, M., Knapen, J., Carraro, A., De Hert, M., 2012a. Associations between sedentary behaviour and metabolic parameters in patients with schizophrenia. Psychiatry Res. 200, 73-78. https://doi.org/10.1016/j.psychres.2012.03.046

Vancampfort, Vansteelandt, K., Scheewe, T., Probst, M., Knapen, J., De Herdt, A., De Hert, M., 2012b. Yoga in schizophrenia: A systematic review of randomised controlled trials. Acta Psychiatr. Scand. https://doi.org/10.1111/j.1600-0447.2012.01865.x

Varambally, S., Gangadhar, B.N., Thirthalli, J., Jagannathan, A., Kumar, S., Venkatasubramanian, G., Muralidhar, D., Subbakrishna, D.K., Nagendra, H.R., 2012. Therapeutic efficacy of add-on yogasana intervention in stabilized outpatient schizophrenia: Randomized controlled comparison with exercise and waitlist. Indian J. Psychiatry 54, 227-232. https://doi.org/10.4103/0019-5545.102414

Ventura, J., Tom, S.R., Jetton, C., Kern, R.S., 2013. Memory functioning and negative symptoms as differential predictors of social problem solving skills in schizophrenia. Schizophr. Res. 143, 307-311. https://doi.org/10.1016/j.schres.2012.10.043

Vera-Garcia, E., Mayoral-Cleries, F., Vancampfort, D., Stubbs, B., Cuesta-Vargas, A.I., 2015. A systematic review of the benefits of physical therapy within a multidisciplinary care approach for people with schizophrenia: An update. Psychiatry Res. 229, 828-839. https://doi.org/10.1016/j.psychres.2015.07.083

Visceglia, E., Lewis, S., 2011. Yoga therapy as an adjunctive treatment for schizophrenia: A randomized, controlled pilot study. J. Altern. Complement. Med. 17, 601-607. https://doi.org/10.1089/acm.2010.0075

Vogel, J.S., van der Gaag, M., Slofstra, C., Knegtering, H., Bruins, J., Castelein, S., 2019. The effect of mind-body and aerobic exercise on negative symptoms in schizophrenia: A meta-analysis. Psychiatry Res. https://doi.org/10.1016/j.psychres.2019.03.012

Vreeland, B., Minsky, S., Menza, M., Radler, D.R., Roemheld-Hamm, B., Stern, R., 2003. A program for managing weight gain associated with atypical antipsychotics. Psychiatr. Serv. 54, 1155-1157. https://doi.org/10.1176/appi.ps.54.8.1155

Wang, P.W., Lin, H.C., Su, C.Y., Chen, M. De, Lin, K.C., Ko, C.H., Yen, C.F., 2018. Effect of aerobic exercise on improving symptoms of individuals with schizophrenia: A single blinded randomized control study. Front. Psychiatry 9. https://doi.org/10.3389/fpsyt.2018.00167

Ward, M.C., White, D.T., Druss, B.G., 2015. A meta-review of lifestyle interventions for cardiovascular risk factors in the general medical population: Lessons for individuals with serious mental illness, in: Journal of Clinical Psychiatry. Physicians Postgraduate Press Inc., pp. e477-e486. https://doi.org/10.4088/JCP.13r08657

Weber, M., Wyne, K., 2006. A cognitive/behavioral group intervention for weight loss in patients treated with atypical antipsychotics. Schizophr. Res. 83, 95-101. https://doi.org/10.1016/j.schres.2006.01.008

Wu, M.K., Wang, C.K., Bai, Y.M., Huang, C.Y., Lee, S. Da, 2007. Outcomes of obese, clozapinetreated inpatients with schizophrenia placed on a six-month diet and physical activity 
program. Psychiatr. Serv. 58, 544-550. https://doi.org/10.1176/ps.2007.58.4.544

Wu, R.R., Zhao, J.P., Jin, H., Shao, P., Fang, M.S., Guo, X.F., He, Y.Q., Liu, Y.J., Chen, J.D., Li, L.H., 2008. Lifestyle intervention and metformin for treatment of antipsychotic-induced weight gain: A randomized controlled trial. JAMA - J. Am. Med. Assoc. 299, 185-193. https://doi.org/10.1001/jama.2007.56-b 
$++$

\begin{tabular}{|c|c|c|c|c|c|c|c|c|c|c|c|c|c|c|c|c|c|c|}
\hline \multirow[t]{2}{*}{$\begin{array}{l}\text { Study } \\
\text { (Country) }\end{array}$} & \multicolumn{2}{|c|}{$\mathrm{N}$ (start) } & \multicolumn{2}{|c|}{$\begin{array}{l}\text { Age, } \\
\text { y (SD) }\end{array}$} & \multicolumn{2}{|c|}{$\begin{array}{l}\text { Gender } \\
\text { (men) }\end{array}$} & \multirow[t]{2}{*}{ Diagnosi (\%)s (y) } & & \multirow[t]{2}{*}{$\begin{array}{l}\text { EG intervention } \\
\text { (format) }\end{array}$} & \multirow[t]{2}{*}{ Freq EG } & \multirow[t]{2}{*}{$\begin{array}{c}\text { Time; } \\
\text { ws }\end{array}$} & \multirow{2}{*}{$\begin{array}{c}\text { CG } \\
\text { intervention } \\
\text { (mode) }\end{array}$} & \multicolumn{2}{|c|}{$\begin{array}{l}\text { Drop- } \\
\text { outs }\end{array}$} & \multirow[t]{2}{*}{$\begin{array}{l}\text { F-Up; } \\
\text { ws }\end{array}$} & \multirow{2}{*}{$\begin{array}{l}\text { Antropometric } \\
\text { and metabolic } \\
\text { outcomes }\end{array}$} & \multirow{2}{*}{$\begin{array}{l}\text { Psychopathology } \\
\text { and clinical } \\
\text { outcomes }\end{array}$} & \multirow{2}{*}{ 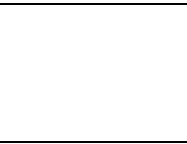 } \\
\hline & EG & CG & EG & CG & EG & CG & & & & & & & EG & CG & & & & \\
\hline & & & & & & & & & & Outpatients & & & & & & & & \\
\hline $\begin{array}{l}\text { Beebe LH. et } \\
\text { al., } 2005 \\
\text { (USA) }\end{array}$ & 6 & 6 & $\begin{array}{l}40- \\
63 y\end{array}$ & $\begin{array}{l}40- \\
63 y\end{array}$ & & & SCZ (100) & 8.6 & $\begin{array}{l}\text { Light AE (group): } \\
\text { treadmill walking }\end{array}$ & $30 \mathrm{~min} \times 3 / \mathrm{w}$ & 16 & $\begin{array}{l}\text { TAU } \\
\text { (indiv) }\end{array}$ & 2 & 0 & No & BMI & PANSS & 6MWT \\
\hline $\begin{array}{l}\text { Acil AA. et al., } \\
2008 \\
\text { (Turkey) }\end{array}$ & 15 & 15 & NS & NS & & & SCZ (100) & 10.3 & $\begin{array}{l}\text { Moderate AE } \\
\quad \text { (group) }\end{array}$ & $45 \mathrm{~min} \times 3 / \mathrm{w}$ & 10 & TAU (indiv) & NS & NS & No & No & SANS; SAPS; BSI & WHOQOL-BREF \\
\hline $\begin{array}{l}\text { Pajonk FG. et } \\
\text { al., 2010 } \\
\text { (Germany) }\end{array}$ & 11 & 13 & $\begin{array}{c}32.9 \\
(10.6)\end{array}$ & $\begin{array}{l}37.4 \\
(8.1)\end{array}$ & 11 & 13 & SCZ (100) & 10.45 & $\begin{array}{c}\text { Moderate } \\
\text { aerobic exercise } \\
\text { (group): cycling }\end{array}$ & $30 \mathrm{~min} \times 3 / \mathrm{w}$ & 12 & $\begin{array}{c}\text { TAU + } \\
\text { football } \\
\text { table }\end{array}$ & 3 & 5 & No & No & $\begin{array}{c}\text { RAVLT; Corsi } \\
\text { direct block span }\end{array}$ & No \\
\hline \multirow[t]{2}{*}{$\begin{array}{l}\text { Varambally S. } \\
\text { et al., } 2012 \\
\text { (India) }\end{array}$} & 47 & 36 & $\begin{array}{c}32.8 \\
(10.0)\end{array}$ & $\begin{array}{l}33.6 \\
(9.5)\end{array}$ & 28 & 27 & SCZ (100) & 9.8 & $\begin{array}{c}\text { MBE: Yoga } \\
\text { (group/indiv) }\end{array}$ & $\begin{array}{c}45 \text { min } \times 25 / \\
1^{\text {st }} \text { month }+ \\
\text { practice at } \\
\text { home next } 3 \\
\text { months }\end{array}$ & 16 & WL (indiv) & 8 & 2 & No & No & PANSS & SOFS \\
\hline & 37 & & $\begin{array}{l}30.6 \\
(7.3)\end{array}$ & & 28 & & & & $\begin{array}{l}\text { Light aerobic } \\
\text { exercise: walking, } \\
\text { jogging and } \\
\text { exercises in } \\
\text { standing and } \\
\text { sitting } \\
\text { postures(group/i } \\
\text { ndiv) }\end{array}$ & $\begin{array}{c}45 \mathrm{~min} \times 25 / \\
1^{\text {st }} \text { month + } \\
\text { practice at } \\
\text { home next } 3 \\
\text { months }\end{array}$ & & & 15 & & & & & \\
\hline $\begin{array}{l}\text { Jayaram N et } \\
\text { al., 2013^ } \\
\text { (India) }\end{array}$ & 15 & 28 & $\begin{array}{l}28.3 \\
(4.7)\end{array}$ & $\begin{array}{l}29.5 \\
(8.2)\end{array}$ & 12 & 7 & SCZ (100) & 6 & $\begin{array}{l}\text { MBE: Yoga } \\
\text { (group) }\end{array}$ & NS & 4 & WL (indiv) & 0 & 16 & No & No & $\begin{array}{l}\text { SAPS; SANS; } \\
\text { TRENDS }\end{array}$ & SOFS \\
\hline $\begin{array}{l}\text { Svatkova A et } \\
\text { al. } 2015 \\
\text { (Netherlands) }\end{array}$ & 16 & 17 & $\begin{array}{l}28.8 \\
(7.4)\end{array}$ & $\begin{array}{l}31.3 \\
(8.2)\end{array}$ & 13 & 14 & SCZ (100) & 7.4 & $\begin{array}{l}\text { Mixed exercise: } \\
\text { Aerobic and } \\
\text { anaerobic } \\
\text { (group) }\end{array}$ & $60 \mathrm{~min} \times 2 / \mathrm{w}$ & 24 & Life as usual & NS & NS & No & BMI & PANSS & $\begin{array}{c}\text { W peak; VO2; } \\
\text { HR }\end{array}$ \\
\hline $\begin{array}{l}\text { Isuru LA et al., } \\
2015 \\
\text { (Sri Lanka) }\end{array}$ & 33 & 40 & $\begin{array}{l}38.8 \\
(9.5)\end{array}$ & $\begin{array}{l}41.9 \\
(9.8)\end{array}$ & 26 & 30 & SCZ (100) & & $\begin{array}{l}\text { Mixed exercise: } \\
\text { MBE (yoga) + } \\
\text { aerobic exercise } \\
\text { (dance) }\end{array}$ & $\begin{array}{c}360 \min x \\
1 / w\end{array}$ & 8 & OT (group) & NS & NS & No & No & PANSS & No \\
\hline
\end{tabular}




\begin{tabular}{|c|c|c|c|c|c|c|c|c|c|c|c|c|c|c|c|c|c|c|}
\hline \multirow[t]{2}{*}{$\begin{array}{l}\text { Study } \\
\text { (Country) }\end{array}$} & \multicolumn{2}{|c|}{$\mathrm{N}$ (start) } & \multicolumn{2}{|c|}{$\begin{array}{l}\text { Age, } \\
\text { y (SD) }\end{array}$} & \multicolumn{2}{|c|}{$\begin{array}{l}\text { Gender } \\
\text { (men) }\end{array}$} & \multirow[t]{2}{*}{ Diagnosi (\%)s (y) } & & \multirow[t]{2}{*}{$\begin{array}{l}\text { EG intervention } \\
\text { (format) }\end{array}$} & \multirow[t]{2}{*}{ Freq EG } & \multirow[t]{2}{*}{$\begin{array}{l}\text { Time; } \\
\text { ws }\end{array}$} & \multirow{2}{*}{$\begin{array}{c}\text { CG } \\
\text { intervention } \\
\text { (mode) }\end{array}$} & \multicolumn{2}{|c|}{$\begin{array}{l}\text { Drop- } \\
\text { outs }\end{array}$} & \multirow[t]{2}{*}{$\begin{array}{l}\text { F-Up; } \\
\text { ws }\end{array}$} & \multirow{2}{*}{$\begin{array}{l}\text { Antropometric } \\
\text { and metabolic } \\
\text { outcomes }\end{array}$} & \multirow{2}{*}{$\begin{array}{l}\text { Psychopathology } \\
\text { and clinical } \\
\text { outcomes }\end{array}$} & \\
\hline & EG & CG & EG & CG & EG & CG & & & & & & & EG & CG & & & & \\
\hline \multirow[t]{2}{*}{$\begin{array}{l}\text { Silva B et al., } \\
2015 \\
\text { (Brasil) }\end{array}$} & 17 & 16 & $\begin{array}{l}33.5 \\
(2.6)\end{array}$ & $\begin{array}{c}33.4 \\
(12.2)\end{array}$ & 17 & 16 & SCZ (100) & 13.01 & $\begin{array}{l}\text { Mixed exercise: } \\
\text { Aerobic and } \\
\text { anaerobic } \\
\text { exercise (group) }\end{array}$ & $60 \mathrm{~min} \times 2 / \mathrm{w}$ & 20 & $\begin{array}{l}\text { TAU + } \\
\text { mínimum } \\
\text { exercise at } \\
\text { treadmild }\end{array}$ & 8 & 3 & No & No & PANSS; CDS & SF-36; 1RMT \\
\hline & 014 & & $\begin{array}{l}32.9 \\
(2.3) \\
\end{array}$ & & 14 & & & & $\begin{array}{c}\text { Anaerobic } \\
\text { exercise (group) }\end{array}$ & & & $\begin{array}{c}\text { and load } \\
\text { (group) }\end{array}$ & 2 & & & & & \\
\hline \multirow[t]{2}{*}{$\begin{array}{l}\text { Hsu CC. et al., } \\
2016 \\
\text { (Taiwan) }\end{array}$} & 18 & 15 & $\begin{array}{c}42.7 \\
(11.3)\end{array}$ & $\begin{array}{c}45.7 \\
(11.0)\end{array}$ & 13 & 12 & SCZ (100) & 25 & $\begin{array}{c}\text { Moderate } \\
\text { aerobic exercise } \\
\text { (group): dancing }\end{array}$ & $\begin{array}{l}50 \min x \\
2 / w\end{array}$ & 8 & TAU (indiv) & 0 & 0 & Yes & Weight & No & $\mathrm{HR}$ \\
\hline & 18 & 15 & - & - & - & & SCZ (100) & & - & - & - & - & 0 & 0 & 4 & Weight & No & $\mathrm{HR}$ \\
\hline \multirow{4}{*}{$\begin{array}{l}\text { Bhatia T. et al., } \\
2017 \\
\text { (India) }\end{array}$} & 104 & 92 & $\begin{array}{l}34.8 \\
(9.8) \\
\end{array}$ & $\begin{array}{c}35.7 \\
(10.1) \\
\end{array}$ & 62 & 57 & SCZ (100) & & $\begin{array}{l}\text { MBE: Yoga } \\
\text { (group) }\end{array}$ & $60 \mathrm{~min} / \mathrm{d}$ & 3 & TAU (indiv) & 18 & 15 & Yes & No & $\begin{array}{c}\text { SANS; SAPS; } \\
\text { Neurocognition }\end{array}$ & GAF \\
\hline & 90 & & $\begin{array}{l}35.2 \\
(9.5)\end{array}$ & & 62 & & & & $\begin{array}{l}\text { Light aerobic } \\
\text { exercise: walking, } \\
\text { jogging and } \\
\text { exercises in } \\
\text { standing and } \\
\text { sitting postures } \\
\text { (group) } \\
\end{array}$ & $60 \mathrm{~min} / \mathrm{d}$ & & & 15 & & & & (Penn CNB) & \\
\hline & 86 & 77 & - & - & - & - & SCZ (100) & & - & - & - & - & 7 & 21 & 24 & No & $\begin{array}{l}\text { SANS; SAPS; } \\
\text { Neurocognition } \\
\text { (Penn CNB) }\end{array}$ & GAF \\
\hline & 75 & & - & & - & & & & & & & & 25 & & & & & \\
\hline \multirow[t]{2}{*}{$\begin{array}{l}\text { Cheng SL. et al., } \\
2017 \\
\text { (Chine) }\end{array}$} & 26 & 28 & $\begin{array}{c}43.7 \\
(12.7)\end{array}$ & $\begin{array}{l}47.4 \\
(9.7)\end{array}$ & 20 & 22 & SCZ (100) & & $\begin{array}{c}\text { Moderate } \\
\text { aerobic exercise } \\
\text { (group): dancing }\end{array}$ & $60 \mathrm{~min} \times 2 / \mathrm{w}$ & 8 & $\begin{array}{l}\text { TAU (indiv/ } \\
\text { group) }\end{array}$ & 4 & 2 & Yes & Weight; BMI & No & $\begin{array}{c}\text { 1-minute flexed } \\
\text { leg sit-up; sit- } \\
\text { and-reach test; } \\
\text { 3-minute } \\
\text { step test } \\
\end{array}$ \\
\hline & 22 & 26 & - & - & - & - & SCZ (100) & & - & - & - & - & 0 & 0 & 12 & Weight; BMI & No & $\begin{array}{l}\text { 1-minute flexed } \\
\text { leg sit-up; sit- } \\
\text { and-reach test; } \\
\text { 3-minute } \\
\text { step test }\end{array}$ \\
\hline \multirow[t]{2}{*}{$\begin{array}{l}\text { Wang PW et al., } \\
2018 \\
\text { (Taiwan) }\end{array}$} & 33 & 29 & & s & 15 & 15 & SCZ (100) & & $\begin{array}{c}\text { Moderate } \\
\text { aerobic exercise } \\
\text { (group) }\end{array}$ & $40 \mathrm{~min} \times 3 / \mathrm{w}$ & 12 & $\begin{array}{c}\text { TAU + } \\
\text { stretching } \\
\text { (group) } \\
\end{array}$ & 8 & 6 & Yes & No & PANSS & No \\
\hline & 25 & 23 & & s & - & - & SCZ (100) & & - & - & - & - & 0 & 0 & 12 & No & PANSS & No \\
\hline
\end{tabular}




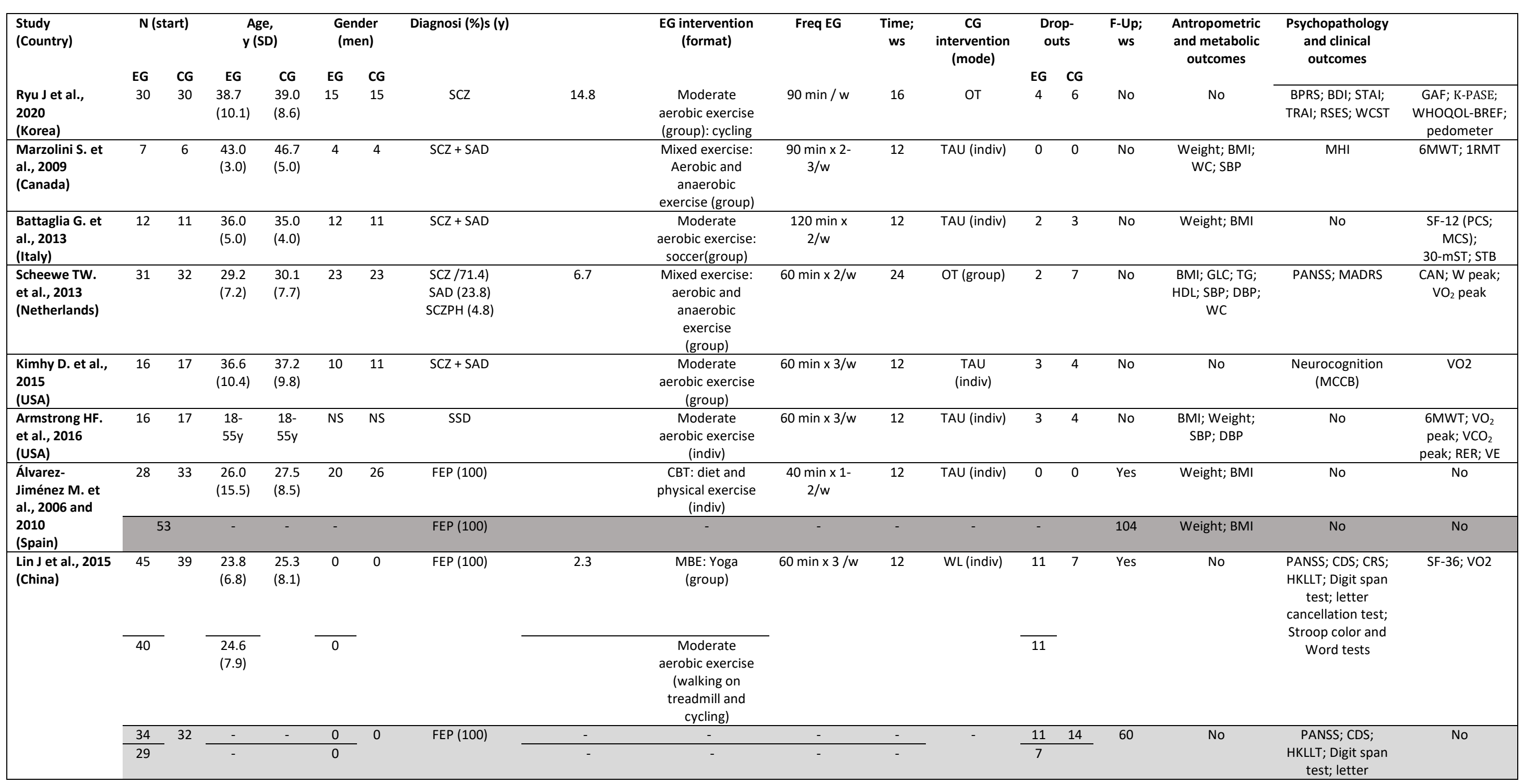




\begin{tabular}{|c|c|c|c|c|c|c|c|c|c|c|c|c|c|c|c|c|c|c|}
\hline \multirow[t]{3}{*}{$\begin{array}{l}\text { Study } \\
\text { (Country) }\end{array}$} & \multicolumn{2}{|c|}{$\mathrm{N}$ (start) } & \multicolumn{2}{|c|}{$\begin{array}{l}\text { Age, } \\
\text { y (SD) }\end{array}$} & \multicolumn{2}{|c|}{$\begin{array}{l}\text { Gender } \\
\text { (men) }\end{array}$} & \multirow[t]{3}{*}{ Diagnosi (\%)s (y) } & & \multirow[t]{2}{*}{$\begin{array}{l}\text { EG intervention } \\
\text { (format) }\end{array}$} & \multirow[t]{2}{*}{ Freq EG } & \multirow[t]{2}{*}{$\begin{array}{l}\text { Time; } \\
\text { ws }\end{array}$} & \multirow{3}{*}{$\begin{array}{c}\text { CG } \\
\text { intervention } \\
\text { (mode) }\end{array}$} & \multicolumn{2}{|c|}{$\begin{array}{l}\text { Drop- } \\
\text { outs }\end{array}$} & \multirow[t]{3}{*}{$\begin{array}{l}\text { F-Up; } \\
\text { ws }\end{array}$} & \multirow{2}{*}{$\begin{array}{c}\text { Antropometric } \\
\text { and metabolic } \\
\text { outcomes }\end{array}$} & \multirow{2}{*}{$\begin{array}{l}\text { Psychopathology } \\
\text { and clinical } \\
\text { outcomes }\end{array}$} & \\
\hline & EG & CG & EG & CG & EG & CG & & & & & & & EG & CG & & & & \\
\hline & & & & & & & & & & & & & & & & & $\begin{array}{l}\text { cancellation test; } \\
\text { Stroop color and } \\
\text { Word tests }\end{array}$ & \\
\hline $\begin{array}{l}\text { Fisher E et al., } \\
2020 \\
\text { (UK) }\end{array}$ & 11 & 11 & $\begin{array}{l}23.5 \\
(3.8)\end{array}$ & $\begin{array}{l}26.1 \\
(5.7)\end{array}$ & 11 & 11 & FEP (100) & 3 & $\begin{array}{l}\text { Moderate } \\
\text { aerobic exercise: } \\
\text { running, } \\
\text { swimming, } \\
\text { cycling, tennis }\end{array}$ & $\begin{array}{c}50 \min _{3 / w} \times 2- \\
-1\end{array}$ & 12 & TAU (indiv) & 2 & 0 & No & Weight & PANSS & $\begin{array}{c}\text { WHOQOL; } \\
\text { IPAQ; WHODAS }\end{array}$ \\
\hline $\begin{array}{l}\text { Ikai S et al., } \\
2013 \\
\text { (Japan) }\end{array}$ & 25 & 24 & $\begin{array}{l}54.8 \\
(9.0)\end{array}$ & $\begin{array}{l}51.5 \\
(15.1)\end{array}$ & 16 & 16 & SSD & 26.1 & $\begin{array}{l}\text { MBE: Yoga } \\
\text { (group) }\end{array}$ & $60 \min \times 1 / w$ & 8 & $\begin{array}{l}\text { Day-care } \\
\text { program } \\
\text { (group) }\end{array}$ & 2 & 3 & Yes* & No & PANSS & EQ5D; FACT-Sz \\
\hline $\begin{array}{l}\text { Ikai S et al., } \\
2014 \\
\text { (Japan) }\end{array}$ & 25 & 25 & $\begin{array}{l}53.5 \\
(9.9)\end{array}$ & $\begin{array}{c}48.2 \\
(12.3)\end{array}$ & 16 & 17 & SSD & 25 & $\begin{array}{l}\text { MBE: Yoga } \\
\text { (group) }\end{array}$ & $60 \mathrm{~min} \times 1 / \mathrm{w}$ & 8 & $\begin{array}{l}\text { Day-care } \\
\text { program } \\
\text { (group) }\end{array}$ & 7 & 7 & Yes* & $\begin{array}{l}\text { Glc; TG; HDL; } \\
\text { LDL; CL }\end{array}$ & PANSS & EQ5D; FACT-Sz \\
\hline $\begin{array}{l}\text { Holt R. et al., } \\
2019 \\
\text { (UK) }\end{array}$ & 207 & 205 & $\begin{array}{c}40.0 \\
(11.3)\end{array}$ & $\begin{array}{c}40.1 \\
(11.5)\end{array}$ & 115 & 95 & $\begin{array}{l}\text { FEP (15.3) + SCZ } \\
(68.7)+\text { SAD (16) }\end{array}$ & & $\begin{array}{l}\text { CBT: diet and } \\
\text { physical activity } \\
\text { (group) }\end{array}$ & $\begin{array}{l}\text { 1) CBT (0- } \\
4 w): 150 \text { min } \\
\times 1 / w \text {; } \\
\text { 2) Phone call } \\
\text { (5-52w) } 10 \\
\text { min } \times 1 / 2 w ; \\
\text { 3) Remainder } \\
\text { CBT: } 150 \text { min } \\
\text { at } 4-7-10 \\
\text { months. }\end{array}$ & 52 & TAU (indiv) & 40 & 32 & No & $\begin{array}{c}\text { Weight; BMI; } \\
\text { WC; SBP; DBP; } \\
\text { GLC; CL; HDL; } \\
\text { TG }\end{array}$ & $\begin{array}{c}\text { BPRS; BIPQ; PHQ- } \\
9\end{array}$ & $\begin{array}{c}\text { EQ5D; } \\
\text { accelerometer }\end{array}$ \\
\hline $\begin{array}{l}\text { Lovell K et al. } \\
2014 \\
\text { (UK) }\end{array}$ & 54 & 51 & $\begin{array}{l}25.6 \\
(5.5)\end{array}$ & $\begin{array}{l}25.9 \\
(6.0)\end{array}$ & 33 & 30 & $\begin{array}{c}\text { FEP: SCZ (5.7) + } \\
\text { SAD (1.9) + SSD } \\
(77.1)+\text { NS (15.3) }\end{array}$ & & $\begin{array}{c}\text { Psychoeducation: } \\
\text { diet and physical } \\
\text { activity (indiv) + } \\
\text { optional } \\
\text { exercises (group) }\end{array}$ & $\begin{array}{c}7 \text { sessions } \\
\text { over } 6 \\
\text { months, with } \\
\text { a "booster" } \\
\text { session at } 9 \text { - } \\
10 \text { months. }\end{array}$ & 52 & TAU (indiv) & 5 & 7 & No & $\begin{array}{l}\text { Weight; BMl; } \\
\text { WC }\end{array}$ & CDS; BARS & $\begin{array}{l}\text { IPAQ; SF-36; } \\
\text { EQ5D. }\end{array}$ \\
\hline \multirow[t]{2}{*}{$\begin{array}{l}\text { Rotatori A. \& } \\
\text { Fox R. } 1980 \\
\text { (USA) }\end{array}$} & 7 & 7 & $\begin{array}{l}36.7 \\
(8.0)\end{array}$ & $\begin{array}{l}35.0 \\
(8.2)\end{array}$ & 3 & 3 & SCZ (100) & & $\begin{array}{l}\text { BT: diet and } \\
\text { physical activity } \\
\text { (indiv) }\end{array}$ & $1 / w$ & 14 & WL (indiv) & 0 & 0 & Yes & Weight & No & No \\
\hline & 7 & 7 & - & - & - & - & SCZ (100) & & - & - & - & - & 3 & 2 & 16 & Weight & No & No \\
\hline $\begin{array}{l}\text { McCreadie R. et } \\
\text { al., } 2005\end{array}$ & 32 & 33 & \multicolumn{2}{|c|}{$45(13)$} & \multicolumn{2}{|c|}{72} & SCZ (100) & 21 & $\begin{array}{c}\text { Fruits and } \\
\text { vegetables free + }\end{array}$ & $\begin{array}{l}5 \text { free } \\
\text { portions }\end{array}$ & 24 & TAU (indiv) & 0 & 6 & Yes & $\begin{array}{l}\text { BMI; GLC; CT; } \\
\text { HDL; CVR; }\end{array}$ & PANSS & $\begin{array}{l}\text { Eating habits } \\
\text { section of the }\end{array}$ \\
\hline
\end{tabular}




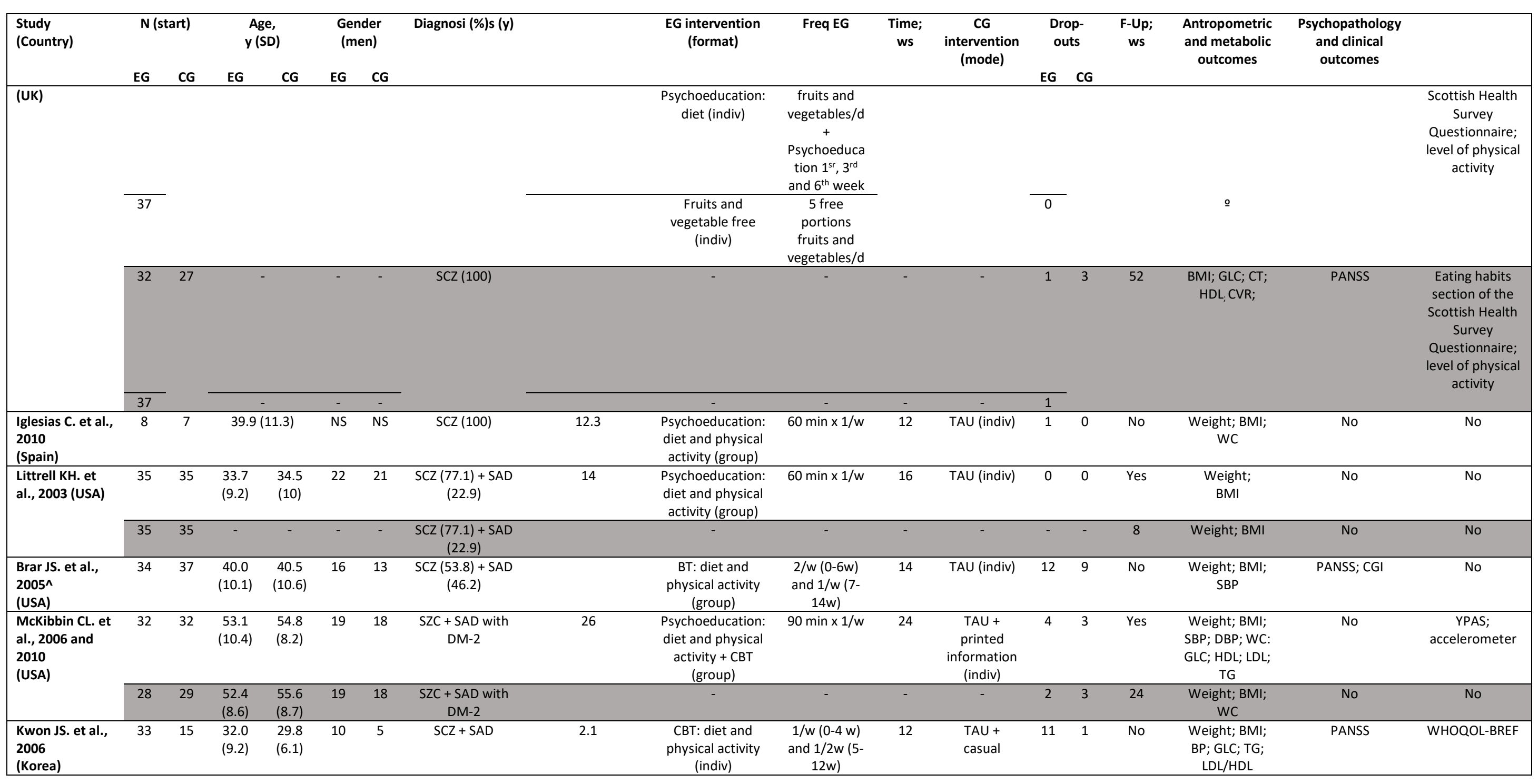




\begin{tabular}{|c|c|c|c|c|c|c|c|c|c|c|c|c|c|c|c|c|c|}
\hline \multirow[t]{2}{*}{$\begin{array}{l}\text { Study } \\
\text { (Country) }\end{array}$} & \multicolumn{2}{|c|}{$\mathbf{N}$ (start) } & \multicolumn{2}{|c|}{$\begin{array}{l}\text { Age, } \\
\text { y (SD) }\end{array}$} & \multicolumn{2}{|c|}{$\begin{array}{l}\text { Gender } \\
\text { (men) }\end{array}$} & \multirow[t]{2}{*}{ Diagnosi (\%)s (y) } & \multirow[t]{2}{*}{$\begin{array}{l}\text { EG intervention } \\
\text { (format) }\end{array}$} & \multirow[t]{2}{*}{ Freq EG } & \multirow[t]{2}{*}{$\begin{array}{l}\text { Time; } \\
\text { ws }\end{array}$} & \multirow{2}{*}{$\begin{array}{c}\text { CG } \\
\text { intervention } \\
\text { (mode) }\end{array}$} & \multicolumn{2}{|c|}{$\begin{array}{l}\text { Drop- } \\
\text { outs }\end{array}$} & \multirow[t]{2}{*}{$\begin{array}{l}\text { F-Up; } \\
\text { ws }\end{array}$} & \multirow{2}{*}{$\begin{array}{c}\text { Antropometric } \\
\text { and metabolic } \\
\text { outcomes }\end{array}$} & \multirow{2}{*}{$\begin{array}{l}\text { Psychopathology } \\
\text { and clinical } \\
\text { outcomes }\end{array}$} & \\
\hline & EG & CG & EG & CG & EG & CG & & & & & & EG & CG & & & & \\
\hline & & & & & & & & & & & $\begin{array}{l}\text { counselling } \\
\text { (indiv) }\end{array}$ & & & & & & \\
\hline $\begin{array}{l}\text { Scocco P. et al., } \\
2006 \\
\text { (Italy) }\end{array}$ & 9 & 10 & $\begin{array}{l}51.7 \\
(12.4)\end{array}$ & $\begin{array}{l}39.2 \\
(9.9)\end{array}$ & 3 & 8 & $\begin{array}{c}\text { SAD (25) + SCZ } \\
\text { (75) }\end{array}$ & $\begin{array}{c}\text { Psychoeducation: } \\
\text { diet and physical } \\
\text { activity + } \\
\text { nutricionist } \\
\text { (indiv) }\end{array}$ & $1 / 2 w$ & 8 & WL (indiv) & 0 & 2 & No & Weight; & No & No \\
\hline $\begin{array}{l}\text { Weber M. \& } \\
\text { Wyne K., } 2006 \\
\text { (USA) }\end{array}$ & 8 & 9 & $\begin{array}{l}18- \\
65 y\end{array}$ & $\begin{array}{l}18- \\
65 y\end{array}$ & 3 & 2 & $S Z C+S A D$ & $\begin{array}{l}\text { CBT: diet and } \\
\text { physical activity } \\
\text { (group) }\end{array}$ & $60 \min \times 1 / w$ & 16 & TAU (indiv) & 0 & 2 & No & Weight; BMI & No & No \\
\hline \multirow[t]{2}{*}{$\begin{array}{l}\text { Gaitero AM. et } \\
\text { al., 2007 } \\
\text { (Spain) }\end{array}$} & 7 & 8 & $\begin{array}{l}43.9 \\
(6.9)\end{array}$ & $\begin{array}{l}38.6 \\
(6.7)\end{array}$ & 3 & 4 & $\begin{array}{c}\text { SZC (73.3)+ SAD } \\
(6.7)+ \\
\text { NS (20) }\end{array}$ & $\begin{array}{c}\text { CBT + } \\
\text { Psychoeducation: } \\
\text { diet and physical } \\
\text { activity (group) }\end{array}$ & $90 \min \times 1 / w$ & 12 & TAU (indiv) & NS & NS & Yes & BMI & No & No \\
\hline & 7 & 8 & - & - & - & - & $\begin{array}{c}\text { SZC (73.3) + SAD } \\
(6.7)+ \\
\text { NS (20) }\end{array}$ & - & - & - & - & NS & NS & 12 & BMI & No & No \\
\hline $\begin{array}{l}\text { Beebe LH. et } \\
\text { al., 2010 } \\
\text { (USA) }\end{array}$ & 48 & 49 & 46.9 & 2.0) & 23 & 28 & $\begin{array}{c}\mathrm{SAD}(71.1)+\mathrm{SCZ} \\
(28.9)\end{array}$ & $\begin{array}{l}\text { Psychoeducation: } \\
\text { diet and physical } \\
\text { activity }+\mathrm{Ml}+ \\
\text { printed } \\
\text { information } \\
\text { (group) }\end{array}$ & $60 \min \times 1 / w$ & 4 & TAC (group) & 13 & 9 & No & No & No & SEE; OEES \\
\hline \multirow[t]{2}{*}{$\begin{array}{l}\text { Beebe LH. et } \\
\text { al., } 2011 \text { and } \\
2013 \\
\text { (USA) }\end{array}$} & 48 & 49 & 46.9 & 2.0) & 23 & 28 & $\begin{array}{c}\text { SCZ (28.9) + SAD } \\
(71.1)\end{array}$ & $\begin{array}{c}\text { Psychoeducation: } \\
\text { diet and physical } \\
\text { activity }+\mathrm{Ml}+ \\
\text { printed } \\
\text { information } \\
\text { (group) } \\
\end{array}$ & $60 \min \times 1 / w$ & 4 & TAC (group) & 11 & 7 & Yes & No & No & $\begin{array}{l}\text { Minutes walked } \\
\text { per month; }\end{array}$ \\
\hline & 11 & 11 & 48.1 & 13.3) & 6 & 6 & $\begin{array}{c}S C Z(36)+S A D \\
\text { (74) }\end{array}$ & - & - & - & - & - & - & 88 & No & No & $\begin{array}{c}\text { Steps and } \\
\text { distance walked } \\
\text { per day; } \\
\text { pedometer }\end{array}$ \\
\hline \multirow[t]{2}{*}{$\begin{array}{l}\text { Attux C. et al., } \\
2013 \\
\text { (Brazil) }\end{array}$} & 81 & 79 & $\begin{array}{l}36.2 \\
(9.9)\end{array}$ & $\begin{array}{c}38.3 \\
(10.7)\end{array}$ & 50 & 46 & $\begin{array}{c}\text { SCZ }(90.4)+N S \\
(9.6)\end{array}$ & $\begin{array}{c}\text { Psychoeducation: } \\
\text { diet and physical } \\
\text { activity (group) + } \\
\text { BT }\end{array}$ & $60 \min \times 1 / w$ & 12 & TAU (indiv) & 21 & 13 & Yes & $\begin{array}{c}\text { Weight; BMI; } \\
\text { WC; GLC; SBP; } \\
\text { DBP; CL; HDL; } \\
\text { LDL; TG }\end{array}$ & RSES & $\begin{array}{c}\text { ILSS; IPAQ; } \\
\text { WHOQOL-BREF; }\end{array}$ \\
\hline & 44 & 41 & - & - & - & - & $\mathrm{SCZ}+\mathrm{NS}$ & - & - & - & - & - & - & 12 & Weight & No & No \\
\hline
\end{tabular}




\begin{tabular}{|c|c|c|c|c|c|c|c|c|c|c|c|c|c|c|c|c|c|c|}
\hline \multirow[t]{2}{*}{$\begin{array}{l}\text { Study } \\
\text { (Country) }\end{array}$} & \multicolumn{2}{|c|}{$\mathrm{N}$ (start) } & \multicolumn{2}{|c|}{$\begin{array}{l}\text { Age, } \\
\text { y (SD) }\end{array}$} & \multicolumn{2}{|c|}{$\begin{array}{l}\text { Gender } \\
\text { (men) }\end{array}$} & \multirow[t]{2}{*}{ Diagnosi (\%)s (y) } & & \multirow[t]{2}{*}{$\begin{array}{l}\text { EG intervention } \\
\text { (format) }\end{array}$} & \multirow[t]{2}{*}{ Freq EG } & \multirow[t]{2}{*}{$\begin{array}{c}\text { Time; } \\
\text { ws }\end{array}$} & \multirow{2}{*}{$\begin{array}{c}\text { CG } \\
\text { intervention } \\
\text { (mode) }\end{array}$} & \multicolumn{2}{|c|}{$\begin{array}{l}\text { Drop- } \\
\text { outs }\end{array}$} & \multirow[t]{2}{*}{$\begin{array}{l}\text { F-Up; } \\
\text { ws }\end{array}$} & \multirow{2}{*}{$\begin{array}{c}\text { Antropometric } \\
\text { and metabolic } \\
\text { outcomes }\end{array}$} & \multirow{2}{*}{$\begin{array}{l}\text { Psychopathology } \\
\text { and clinical } \\
\text { outcomes }\end{array}$} & \multirow{4}{*}{$\begin{array}{c}\mathrm{HR} ; \mathrm{VO}_{2} \text { peak; } \\
\text { FEF; Physical } \\
\text { Activity Scale; } \\
\text { MANSA; EQ5D } \\
\text { GAF }\end{array}$} \\
\hline & EG & CG & EG & CG & EG & CG & & & & & & & EG & CG & & & & \\
\hline \multirow[t]{2}{*}{$\begin{array}{l}\text { Speyer H. et al., } \\
2016 \\
\text { (Denmark) }\end{array}$} & 138 & 142 & $\begin{array}{c}37.8 \\
(12.6)\end{array}$ & $\begin{array}{l}39.5 \\
(12.8)\end{array}$ & 62 & 60 & $\begin{array}{c}\mathrm{SCZ}(88.4)+\mathrm{NS} \\
(11.6)\end{array}$ & & $\mathrm{BT}$ (indiv) & $1 / w$ & 52 & $\begin{array}{l}\text { Coordinatio } \\
n \text { with } \\
\text { primary care } \\
+ \text { TAU } \\
\text { (indiv) }\end{array}$ & 18 & 21 & No & $\begin{array}{l}\text { Weight; BMI; } \\
\text { WC; SBP; HDL; } \\
\text { LDL; TG; 10- } \\
\text { year-CVR }\end{array}$ & SAPS; SANS; BACS & \\
\hline & & 148 & & $\begin{array}{c}38.5 \\
(11.8)\end{array}$ & & 67 & & & & & & TAU (indiv) & & 21 & & & & \\
\hline \multirow[t]{2}{*}{$\begin{array}{l}\text { Sugawara N. et } \\
\text { al., } 2018 \\
\text { (Japan) }\end{array}$} & 87 & 85 & $\begin{array}{c}46.6 \\
(10.9)\end{array}$ & $\begin{array}{c}44.0 \\
(10.3)\end{array}$ & 46 & 49 & $S C Z+S A D$ & 18.3 & PEn: diet (indiv) & $\begin{array}{c}30-40 \min x \\
1 / 4 w\end{array}$ & 52 & TAU (indiv) & 26 & 24 & No & $\begin{array}{c}\text { Weight; BMI; } \\
\text { WC; BP; GLC; } \\
\text { TG; HDL }\end{array}$ & No & No \\
\hline & & 93 & $\begin{array}{l}47.6 \\
(9.6)\end{array}$ & 43 & & 43 & & & & & & $\begin{array}{c}\text { TAU + } \\
\text { casual } \\
\text { counselling } \\
\text { by doctor } \\
\text { (indiv) }\end{array}$ & & 26 & & & & \\
\hline $\begin{array}{l}\text { Wu RR. et al., } \\
2008 \\
\text { (China) }\end{array}$ & 32 & 32 & 26.4 & 25.8 & 17 & 16 & FEP (100) & 0.75 & $\begin{array}{c}\text { Psychoeducation: } \\
\text { diet (group) + } \\
\text { Aerobic exercise } \\
\text { (group/indiv) }\end{array}$ & $30 \min \times 7 / w$ & 12 & $\begin{array}{c}\text { Placebo } \\
\text { (inidiv) }\end{array}$ & 3 & 3 & No & BMI; WC; GLC & PANSS & No \\
\hline $\begin{array}{l}\text { Brown C. et al., } \\
2014 \\
\text { (USA) }\end{array}$ & 70 & 66 & $\begin{array}{c}44.4 \\
(11.7)\end{array}$ & $\begin{array}{c}44.9 \\
(10.1)\end{array}$ & 25 & 20 & SCZ (100) & & $\begin{array}{c}\text { Psychoeducation: } \\
\text { diet and physical } \\
\text { activity (group) + } \\
\text { Aerobic exercise } \\
\text { (group) }\end{array}$ & $\begin{array}{l}\text { Intensive: } 0- \\
12 \mathrm{w} \\
\text { maintenance } \\
: 13-24 \mathrm{w} ; \\
\text { intermitent } \\
\text { support: } 25- \\
52 \mathrm{w}\end{array}$ & 52 & TAU (indiv) & 23 & 21 & No & Weight & No & No \\
\hline $\begin{array}{l}\text { Kang R et al., } \\
2016 \\
\text { (China) }\end{array}$ & 118 & 126 & $\begin{array}{c}46.4 \\
(11.9)\end{array}$ & $\begin{array}{c}45.4 \\
(12.3)\end{array}$ & 53 & 63 & SCZ (100) & 20.6 & $\begin{array}{l}\text { BT: Social skill } \\
\text { Training (45 min) } \\
\text { + MBE: Tai-chi } \\
\text { (45 min) (group) }\end{array}$ & $\begin{array}{c}120 \min x \\
2 / 4 w\end{array}$ & 52 & TAU (indiv) & 0 & 0 & No & No & PANSS & WHOQOL-BREF \\
\hline $\begin{array}{l}\text { Jean-Baptiste } \\
\text { M. et al., } 2017 \\
\text { (USA) }\end{array}$ & 9 & 9 & 52.4 & 40.7 & 2 & 7 & $\begin{array}{c}\mathrm{SCZ}(55.6)+\mathrm{SAD} \\
(44.4)\end{array}$ & & $\begin{array}{c}\text { BT (indiv) + } \\
\text { Aerobic exercise } \\
\text { (group) + } \\
\text { Psychoeducation: } \\
\text { diet and physical } \\
\text { activity (group)( }\end{array}$ & $\begin{array}{c}45-60 \min x \\
1 / w\end{array}$ & 16 & WL (indiv) & 1 & 3 & $\begin{array}{l}\text { Yes* } \\
\text { (24) }\end{array}$ & Weight & No & No \\
\hline
\end{tabular}




\begin{tabular}{|c|c|c|c|c|c|c|c|c|c|c|c|c|c|c|c|c|c|c|}
\hline \multirow{3}{*}{$\begin{array}{l}\text { Study } \\
\text { (Country) } \\
\text { Sailer P. et al., } \\
\text { 2015 } \\
\text { (Gernany \& } \\
\text { Switzerland) }\end{array}$} & \multicolumn{2}{|c|}{$\mathrm{N}$ (start) } & \multicolumn{2}{|c|}{$\begin{array}{l}\text { Age, } \\
\text { y (SD) }\end{array}$} & \multicolumn{2}{|c|}{$\begin{array}{l}\text { Gender } \\
\text { (men) }\end{array}$} & \multirow[t]{2}{*}{ Diagnosi (\%)s (y) } & & \multirow[t]{2}{*}{$\begin{array}{l}\text { EG intervention } \\
\text { (format) }\end{array}$} & \multirow[t]{2}{*}{ Freq EG } & \multirow[t]{2}{*}{$\begin{array}{l}\text { Time; } \\
\text { ws }\end{array}$} & \multirow{2}{*}{$\begin{array}{c}\text { CG } \\
\text { intervention } \\
\text { (mode) }\end{array}$} & \multicolumn{2}{|c|}{$\begin{array}{l}\text { Drop- } \\
\text { outs }\end{array}$} & \multirow[t]{2}{*}{$\begin{array}{l}\text { F-Up; } \\
\text { ws }\end{array}$} & \multirow{2}{*}{$\begin{array}{c}\text { Antropometric } \\
\text { and metabolic } \\
\text { outcomes }\end{array}$} & \multirow{2}{*}{$\begin{array}{l}\text { Psychopathology } \\
\text { and clinical } \\
\text { outcomes }\end{array}$} & \\
\hline & EG & CG & & CG & EG & CG & & & & & & & EG & CG & & & & \\
\hline & $\begin{array}{l}19: \\
7+ \\
12\end{array}$ & $\begin{array}{c}17: \\
4+1 \\
3\end{array}$ & 30.9 & 11.4) & 12 & 13 & $\begin{array}{c}\text { FEP }(23.4)+\text { SZC } \\
(42.5)+D D(2.1) \\
+\operatorname{ATP}(10.6)+ \\
\text { SAD (21.4) }\end{array}$ & & $\begin{array}{l}\text { Moderate } \\
\text { AE(group): } \\
\text { jogging }\end{array}$ & $30 \mathrm{~min} \times 2 / \mathrm{w}$ & 4 & $\begin{array}{c}\text { TAU + } \\
\text { informative } \\
\text { test about } \\
\text { physical } \\
\text { activity } \\
\text { (indiv) } \\
\end{array}$ & 4 & 3 & No & No & $\begin{array}{l}\text { PANSS; BDI; } \\
\text { attention }\end{array}$ & IPAQ \\
\hline $\begin{array}{l}\text { Visceglia E. \& } \\
\text { Lewis S. } 2011 \\
\text { (USA) }\end{array}$ & 10 & 8 & $\begin{array}{l}37.4 \\
(13.7)\end{array}$ & $\begin{array}{c}48.1 \\
(11.2)\end{array}$ & 6 & 6 & SCZ (100) & & $\begin{array}{l}\text { MBE: Yoga } \\
\text { (group) }\end{array}$ & $45 \min \times 2 / w$ & 8 & WL (indiv) & NS & NS & No & No & PANSS & WHOQOL-BREF \\
\hline $\begin{array}{l}\text { Kaltsatou A. et } \\
\text { al., 2015 } \\
\text { (Greece) }\end{array}$ & 16 & 15 & $\begin{array}{l}59.5 \\
(19.6)\end{array}$ & $\begin{array}{l}60.4 \\
(8.6)\end{array}$ & 14 & 11 & SCZ (100) & 34.4 & $\begin{array}{c}\text { Moderate } \\
\text { aerobic exercise } \\
\text { (group): dancing }\end{array}$ & $60 \mathrm{~min} \times 3 / \mathrm{w}$ & 32 & TAU (indiv) & 0 & 0 & No & BMI & PANSS & $\begin{array}{c}\text { Q-LES-Q; } \\
\text { 6MWT; sit-to- } \\
\text { stand test; BBS; } \\
\text { lower limbs } \\
\text { strength } \\
\text { testing; hand } \\
\text { grip strength; } \\
\text { GAF }\end{array}$ \\
\hline $\begin{array}{l}\text { Loh S et al., } \\
2015 \\
\text { (Malaa) }\end{array}$ & 52 & 52 & 21.6 & 10.2) & 35 & 39 & SCZ (100) & & $\begin{array}{l}\text { Light aerobic } \\
\text { exercise (group): } \\
\text { walking }\end{array}$ & $\begin{array}{c}3 / w: \\
0-4 w: 30 \\
\text { min; } \\
\text { 5-8w: } 40 \\
\text { min; } \\
\text { 9-12w: } 50 \\
\text { min }\end{array}$ & 12 & TAU (indiv) & 4 & 0 & No & No & PANSS & PSP; SF-36 \\
\hline $\begin{array}{l}\text { Paikkatt B et } \\
\text { al., 2015 } \\
\text { (India) }\end{array}$ & 15 & 15 & 20 & & 15 & 15 & SCZ (100) & & $\begin{array}{l}\text { MBE: Yoga } \\
\text { (group) }\end{array}$ & $90 \min \times 7 / w$ & 4 & TAU (indiv) & NS & NS & No & No & PANSS & No \\
\hline $\begin{array}{l}\text { Ho R. et al., } \\
2016 \\
\text { (Hong Kong) }\end{array}$ & 51 & 49 & $\begin{array}{l}52.4 \\
(9.6)\end{array}$ & $\begin{array}{l}54.7 \\
(8.0)\end{array}$ & 31 & 24 & SCZ (100) & 29.9 & $\begin{array}{l}\text { MBE: Tai-chi } \\
\text { (group) }\end{array}$ & $\begin{array}{c}\text { Class of } 60 \\
\mathrm{~min} / \mathrm{w}+45 \\
\mathrm{~min} \text { twice- } \\
\text { weekly } \\
\text { sessions in- } \\
\text { between } \\
\text { under } \\
\text { guidance of } \\
\text { mental } \\
\end{array}$ & 12 & WL (indiv) & 0 & 1 & Yes & No & $\begin{array}{c}\text { PANSS; PSS; ;WAIS } \\
\text { Digit Span; NES }\end{array}$ & $\begin{array}{c}\text { IADL; Barthel's } \\
\text { ADL }\end{array}$ \\
\hline
\end{tabular}




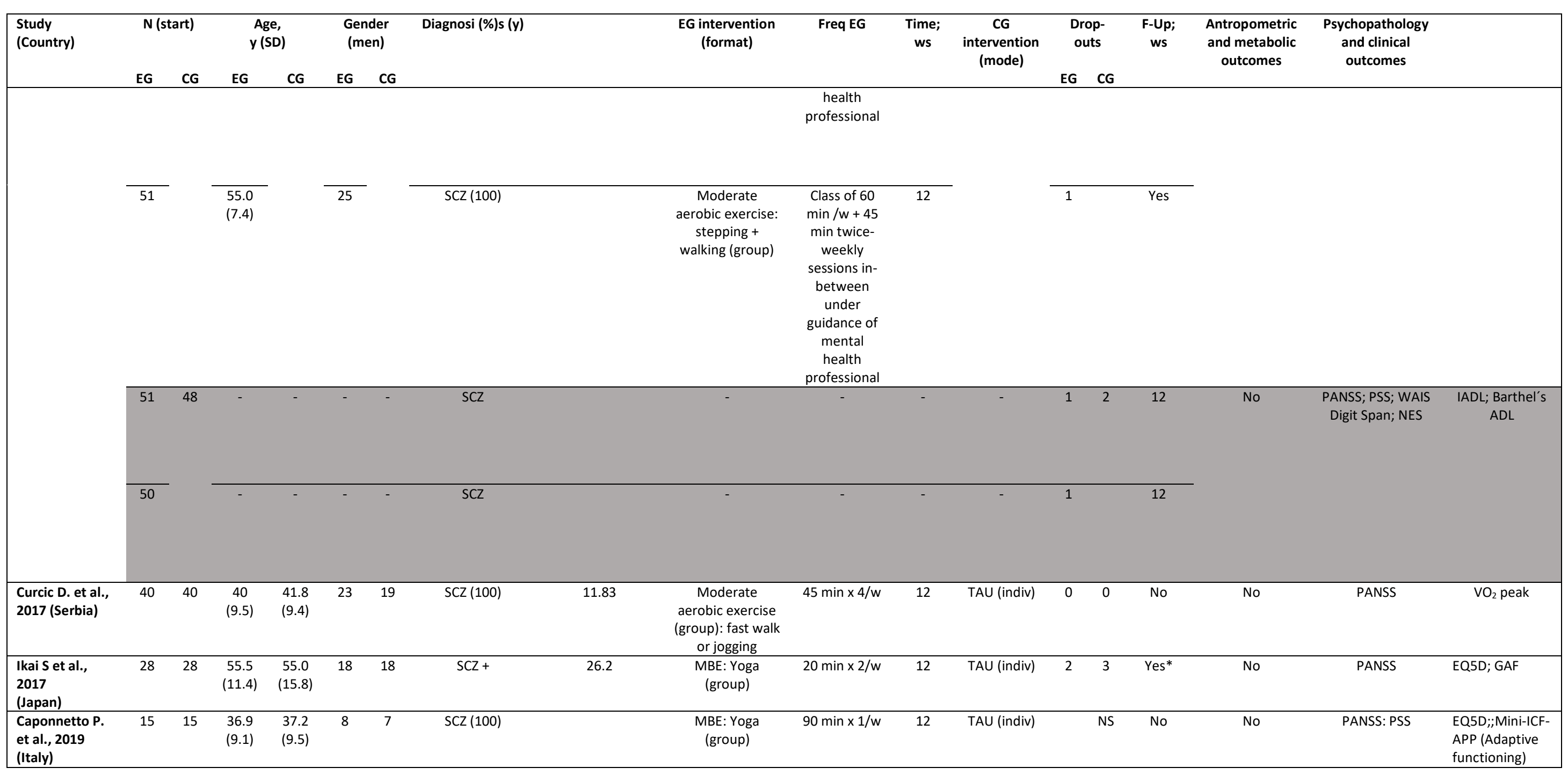




\begin{tabular}{|c|c|c|c|c|c|c|c|c|c|c|c|c|c|c|c|c|c|c|}
\hline \multirow{4}{*}{$\begin{array}{l}\begin{array}{l}\text { Study } \\
\text { (Country) }\end{array} \\
\text { Cordes J. et al., } \\
2014 \\
\text { (Germany) }\end{array}$} & \multicolumn{2}{|c|}{$\mathrm{N}$ (start) } & \multicolumn{2}{|c|}{$\begin{array}{l}\text { Age, } \\
\text { y (SD) }\end{array}$} & \multicolumn{2}{|c|}{$\begin{array}{l}\text { Gender } \\
\text { (men) }\end{array}$} & \multirow[t]{2}{*}{ Diagnosi (\%)s (y) } & & \multirow[t]{2}{*}{$\begin{array}{l}\text { EG intervention } \\
\text { (format) }\end{array}$} & \multirow[t]{2}{*}{ Freq EG } & \multirow[t]{2}{*}{$\begin{array}{c}\text { Time; } \\
\text { ws }\end{array}$} & \multirow{2}{*}{$\begin{array}{c}\text { CG } \\
\text { intervention } \\
\text { (mode) }\end{array}$} & \multicolumn{2}{|c|}{$\begin{array}{l}\text { Drop- } \\
\text { outs }\end{array}$} & \multirow[t]{2}{*}{$\begin{array}{l}\text { F-Up; } \\
\text { ws }\end{array}$} & \multirow{2}{*}{$\begin{array}{c}\text { Antropometric } \\
\text { and metabolic } \\
\text { outcomes }\end{array}$} & \multirow{2}{*}{$\begin{array}{l}\text { Psychopathology } \\
\text { and clinical } \\
\text { outcomes }\end{array}$} & \\
\hline & EG & CG & EG & CG & EG & CG & & & & & & & EG & CG & & & & \\
\hline & 36 & 38 & $\begin{array}{c}38.2 \\
(11.2)\end{array}$ & $\begin{array}{l}35.8 \\
(10.9)\end{array}$ & 15 & 27 & $S C Z+S A D$ & & $\begin{array}{c}\text { BT + } \\
\text { Psychoeducation: } \\
\text { diet and physical } \\
\text { activity (group) }\end{array}$ & $\begin{array}{l}90 \min x \\
1 / 2 w\end{array}$ & 24 & TAU & 23 & 20 & Yes & $\begin{array}{c}\text { Weight; BMI; } \\
\text { WC; SBP; DBP; } \\
\text { GLC; TG; CL }\end{array}$ & PANSS; CGI & No \\
\hline & 13 & 18 & $\begin{array}{l}44.1 \\
(7.5)\end{array}$ & $\begin{array}{c}40.7 \\
(11.7)\end{array}$ & 4 & 11 & $\mathrm{SCZ}+\mathrm{SAD}$ & & - & - & - & - & 2 & 4 & 24 & $\begin{array}{r}\text { Weight; BMI; } \\
\text { WC; SBP; DBP; } \\
\text { GLC; TG; CL }\end{array}$ & PANSS; CGI & No \\
\hline $\begin{array}{l}\text { Wu M et al., } \\
2007 \\
\text { (Taiwan) }\end{array}$ & 28 & 25 & $\begin{array}{l}42.2 \\
(7.5)\end{array}$ & $\begin{array}{l}39.0 \\
(6.7)\end{array}$ & 11 & 11 & SCZ (100) & & $\begin{array}{l}\text { Psychoeducation: } \\
\text { diet (indiv) }+ \\
\text { Aerobic exercise } \\
\text { (group) }\end{array}$ & $60 \mathrm{~min} \times 3 / \mathrm{w}$ & 24 & TAU (indiv) & 0 & 3 & No & $\begin{array}{l}\text { Weight; BMl; } \\
\text { GLC; TG; CL }\end{array}$ & No & No \\
\hline $\begin{array}{l}\text { Methapatara } \\
\text { W. \& } \\
\text { Srisurapanont } \\
\text { M. 2011 } \\
\text { (Thailand) }\end{array}$ & 32 & 32 & $\begin{array}{l}43.2 \\
(9.3)\end{array}$ & $\begin{array}{c}37.6 \\
(10.8)\end{array}$ & 23 & 18 & SCZ (100) & 13.1 & $\begin{array}{c}\text { MI (indiv)+ } \\
\text { Psychoeducation: } \\
\text { diet (group) + } \\
\text { Aerobic exercise } \\
\text { (group/indiv) }\end{array}$ & NS & 12 & $\begin{array}{c}\text { TAU + } \\
\text { printed } \\
\text { information } \\
\text { (indiv.) }\end{array}$ & 4 & 1 & No & $\begin{array}{l}\text { Weight; BMl; } \\
\text { WC }\end{array}$ & No & No \\
\hline \multirow[t]{2}{*}{$\begin{array}{l}\text { Oertel-Knöchel } \\
\text { V. et al., } 2014 \\
\text { (Germany) }\end{array}$} & 8 & 10 & $\begin{array}{c}44.6 \\
(13.8)\end{array}$ & $\begin{array}{l}38.3 \\
(4.5)\end{array}$ & 3 & 5 & SCZ (56.9) † & 10.3 & $\begin{array}{c}\text { Aerobic exercise } \\
+\mathrm{CT} \text { (group) }\end{array}$ & $75 \mathrm{~min} \times 3 / \mathrm{w}$ & 4 & WL (indiv) & NS & NS & No & No & $\begin{array}{c}\text { TMT-A; BACS- } \\
\text { symbol coding } \\
\text { and animal } \\
\text { naming; Category } \\
\text { fluency; WMS-III } \\
\text { SS; LNS; HVLT-R; } \\
\text { BVMT-R; STAI; } \\
\text { RHS; PANSS }\end{array}$ & SF-12 (PCS) \\
\hline & 11 & & $\begin{array}{l}34.9 \\
(9.3) \\
\end{array}$ & & 4 & & & & $\begin{array}{c}\text { Relaxation + CT } \\
\text { (group) }\end{array}$ & $30 \mathrm{~min} \times 3 / \mathrm{w}$ & 4 & & NS & & & & & \\
\hline
\end{tabular}

Table. Characteristics of included studies

1RMT: One Repetition Maximum test; 6MWT: 6-Minutes Walking Test; 30-mST: 30 meter Sprint Test; ADL: Barthel's Activities of Daily Living; AE: Aerobic exercise; AnE: Anaerobic exercise; ATP: Acute and transient psychosis; BACS: Brief Assessment of Cognition; BARS: Brief Adherence Rating Scale; BBS: Berg-Balance Scale Score; BDI: Beck Depression Inventory; BIPQ: Brief Illness Perception Questionnaire; BMI: Body Mass Index; BNSS:: Brief Negative Symptom Scale; BP: Blood Pressure; BPRS: Brief Psychiatric Rating Scale; BSI:Brief Symptom Inventory; BT: Behavior Therapy; BVMT-R: Brief Visuospatial Memory Test-Revised; CAN: The Camberwell Assessment of Need; CBT: Cognitive-behavioral therapy; CDS: Calgary Depression Scale; CNB: Computerized Neurocognitive Battery;CRS: Compliance Rating Scale; EG: Experimental group; CG: Control group; CGI: Clinical Global Impression; CL: cholesterol (total); CVR: Cardiocascular Risk; CT: Cognitive Training; DBP: Diastolic Blood Pressure; DD: Delusional disorder; DM-2: Diabetes Mellitus type 2; EG: Experimental group; EQ5D: European Quality of Life 5 Dimensions; F-up: Follow-up; FACT-Sz: Funtional Assessment for Comprehensive Treatment of Schziophrenia; FEF: Forzed Expiratory Flow; FEP: First Episode of Psychosis; Freq: Frequency; GAF: Global Assessment of Functioning; GLC: Glucose; HDL: High-density lipoprotein; indiv: individual; HKLLT: Hong-Kong List Learning Test; HR: Heart rate; HVLT-R: Hopkins Verbal Learning Test-Revised; IADL: Lawton's Instrumental Activities of Daily Living; ILSS: Independent Living Skills Survey; IPAQ: International Physical Activity Questionnaire; K-PASE: Physical Activity Scale; LDL: Low-density lipoprotein; LNS: Letter- 
Number-Span; MADRS: The Montgomery-Asberg Depression Rating Scale; min: minutes; MANSA: Manchester Short Assessment of Quality of Life;MBE: Mind-Body Exercise; MCCB: MATRICS Consensus Cognitive Battery; MCS-12: mental component summary; MHI: Mental Health Inventory; MI: Motivational Interview; NES: Neurological Evaluation Scale; NIDDM: noninsulin-dependent diabetes mellitus; NS: No Specified; OEES: Outcome expectations for exercise; OT: Occupational Therapy; PANSS: Positive and Negative Syndrome Scale; PCS: physical component summary; PE: Psychoeducation; PHQ: Patient Health Questionnaire; PSP: Personal and Social Performance Scale; PSS: Perceived Stress Scale; Q-LES-Q: Quality of Life Enjoyment and Satisfaction Questionnaire; RAVLT: Rey Auditory Verbal Learning Test; RER: Respiratory Exchange Ratio; RHS: Revised Hallucination Scale; RSES: Rosenberg Self-esteem Scale; SAD: Schizoaffective disorder; SANS: Scale for the Assessment of Negative Symptoms; SAPS: Scale for the Assessment of Positive Symptoms; SBP: Systolic Blood Pressure; SCZ: Schizophrenia; SCZPH: Schizophreniform; SD: Standard Deviation; SEE: Exercise self-efficacy; SF-12: Short Form 12-item Physical and Mental Survey; SF-36: Short Form-36 Health Survey; SOFS: Social and Occupational Functioning Scale; SSD: Schizophrenia Spectrum Disorders; SDS: Sheehan Disability Scale; STB: Slalom test running with ball; STAI: State- Trait-Anxiety-Inventory; t: duration; TAC: Time and attention control; TAU: Treatment as usual; TG: triglycerides; TMT: Trail Making Test; TRAI: Trait Anxiety Inventory; TRENDS: Recognition of emotions in neuripsychiatric disorders; VCO 2 : peak rate of carbon dioxide production; VE: Peak minute ventilation; $\mathrm{VO}_{2}$ peak: peak rate of oxygen uptake; w: week; W peak: Peak power output; WAIS: Wechsler Adult Intelligence Scale; WC: Waist Circumference; WHODAS: World Health Organization Disability Schedule; WHOQOL-BREF: World Health Organization's Quality of Life shorter version; WCST: Wisconsin Card Sorting Test; WL: Waiting List; WMS-III SS: Wechsler Memory Scale Spatial Span; Y: years; YPAS: The Yale Physical Activity Scale.

Shaded rows means follow-up studies.

*Ineligible design for the purpose of the follow-up synthesis.

^Sample contains outpatient and stable long-term inpatients

†A Major Depressive Dissorder sample was not included in the synthesis 


\begin{tabular}{|c|c|c|c|c|c|c|}
\hline \multirow[t]{2}{*}{ Study } & \multicolumn{2}{|c|}{ Selection bias } & \multirow{2}{*}{$\begin{array}{c}\text { Reporting } \\
\text { bias }\end{array}$} & \multirow{2}{*}{$\begin{array}{c}\text { Detection } \\
\text { bias }\end{array}$} & \multirow[t]{2}{*}{ Attrition bias } & \multirow[t]{2}{*}{ Bias score } \\
\hline & $\begin{array}{c}\text { Random } \\
\text { sequence } \\
\text { generation }\end{array}$ & $\begin{array}{c}\text { Allocation } \\
\text { concealment }\end{array}$ & & & & \\
\hline Beebe LH. et al., 2005 & Low & Unclear & High & Unclear & Low & 2 \\
\hline Acil AA. et al., 2008 & Low & High & Low & High & Unclear & 2 \\
\hline Pajonk FG. et al., 2010 & Low & Low & Low & Low & Low & 5 \\
\hline Varambally S. et al., 2012 & Low & Low & Unclear & Low & High & 3 \\
\hline Jayaram N. et al., 2013 & Unclear & Unclear & Unclear & Unclear & High & 0 \\
\hline Svatkova A. et al., 2015 & Low & Low & Low & High & Low & 4 \\
\hline Isuru LAA. et al., 2015 & Unclear & Unclear & High & Low & Low & 2 \\
\hline Silva B et al., 2015 & Low & Low & Low & Low & Low & 5 \\
\hline Hsu CC. et al., 2016 & Low & Unclear & Low & Low & Low & 4 \\
\hline Bhatia T. et al., 2017 & Low & Low & Low & High & Unclear & 3 \\
\hline Cheng SL. et al., 2017 & Low & Unclear & Unclear & Low & Low & 3 \\
\hline Wang PW. et al., 2018 & Unclear & Unclear & Low & Low & Unclear & 2 \\
\hline Ryu J et al., 2020 & Low & Low & Low & Low & Low & 5 \\
\hline Marzolini S. et al., 2009 & Low & Low & Low & Low & Low & 5 \\
\hline Battaglia G. et al., 2013 & Low & Low & Unclear & Low & Low & 4 \\
\hline Scheewe TW. et al., 2013 & Low & Low & Low & High & Low & 4 \\
\hline Kimhy D. et al., 2015 & Low & Low & Low & Low & Low & 5 \\
\hline Armstrong HF. et al., 2016 & Low & Low & Low & Low & Low & 5 \\
\hline Álvarez-Jiménez M. et al., 2006 & Low & Low & Low & Low & Low & 5 \\
\hline Álvarez-Jiménez M. et al., 2010 (follow-up) & Low & Low & Unclear & Low & Low & 4 \\
\hline Lin J. et al., 2015 & Low & Low & Low & Low & Low & 5 \\
\hline Fisher E. et al., 2020 & Low & High & Low & High & Low & 3 \\
\hline Ikai S. et al., 2013 & Low & Low & Unclear & Low & Low & 4 \\
\hline Ikai S. et al., 2014 & Low & Low & Low & Low & Low & 5 \\
\hline Holt R. et al., 2019 & Low & Low & Low & Low & Low & 5 \\
\hline Rotatori A. \& Fox R. 1980 & Low & Unclear & High & Low & Low & 3 \\
\hline McCreadie R. et al., 2005 & High & High & Unclear & Unclear & Low & 1 \\
\hline Iglesias C. et al., 2010 & Low & Unclear & Low & Low & Low & 4 \\
\hline Littrell KH. et al., 2003 & Low & Unclear & Low & Low & Low & 4 \\
\hline Brar JS. et al., 2005 & Low & Unclear & Low & Unclear & Low & 3 \\
\hline McKibbin CL. et al., 2006 & Low & Unclear & Low & Unclear & Low & 3 \\
\hline McKibbin CL. et al., 2010 (follow-up) & Low & Unclear & Low & Low & Low & 4 \\
\hline Kwon JS. et al., 2006 & Low & Unclear & Low & Unclear & Low & 3 \\
\hline Scocco P. et al., 2006 & Low & High & High & Low & Low & 3 \\
\hline Weber M. \& Wyne K., 2006 & Low & Low & Low & Low & Low & 5 \\
\hline Gaitero AM. et al., 2007 & Unclear & Unclear & High & Low & High & 1 \\
\hline Beebe LH. et al., 2010 & Low & Unclear & Low & Unclear & Low & 3 \\
\hline Beebe LH. et al., 2011 & Low & Unclear & Low & Unclear & Low & 3 \\
\hline Beebe LH et al., 2013 (follow-up) & Low & Unclear & Low & Low & Low & 4 \\
\hline Attux C. et al., 2013 & Low & Low & Low & Low & Low & 5 \\
\hline Speyer H. et al., 2016 & Low & Low & High & Low & Low & 4 \\
\hline Sugawara N. et al., 2018 & Low & High & Low & Low & Low & 4 \\
\hline Lovell K et al.2014 & Low & Low & Low & Low & Low & 5 \\
\hline Wu RR et al., 2008 & Low & Low & Low & Low & Low & 5 \\
\hline Brown C. et al., 2014 & Low & Unclear & Low & Low & Low & 4 \\
\hline Kang R et al., 2016 & Low & Low & Low & Low & Low & 5 \\
\hline Jean-Baptiste M. et al., 2017 & Low & Unclear & High & Low & Low & 3 \\
\hline Sailer P. et al., 2015 & Low & Low & High & Low & Low & 4 \\
\hline Visceglia E. \& Lewis S. 2011 & Low & Unclear & Low & Low & Low & 4 \\
\hline Kaltsatou A. et al., 2015 & Unclear & Low & Low & Low & Low & 4 \\
\hline Loh S. et al., 2015 & Low & High & Low & High & Low & 3 \\
\hline Paikkatt E. et al., 2015 & Unclear & Unclear & High & Unclear & Unclear & 0 \\
\hline Ho R. et al., 2016 & Low & Unclear & Unclear & High & Unclear & 1 \\
\hline Curcic D. et al., 2017 & Unclear & Unclear & Unclear & High & Unclear & 0 \\
\hline Ikai S. et al., 2017 & Low & Low & Low & Low & Low & 5 \\
\hline Caponetto P. et al., 2019 & Low & Unclear & High & Unclear & Unclear & 1 \\
\hline Cordes J. et al., 2014 & Low & Unclear & Low & Unclear & Low & 3 \\
\hline Wu M et al., 2007 & Low & Unclear & Low & Low & Low & 4 \\
\hline Methapatara W. \& Srisurapanont M. 2011 & Low & High & Low & Low & Low & 4 \\
\hline Oertel-Knöchel V. et al., 2014 & Low & Low & Low & Unclear & Low & 4 \\
\hline
\end{tabular}

Table 2. Risk of bias of included studies following the Cochrane handbook recommendations: Low risk: the investigators describe a random component for the considered risk Unclear: insufficient information to permit judgment of "Low risk" or "High risk" High risk: the investigators describe a non-random component 


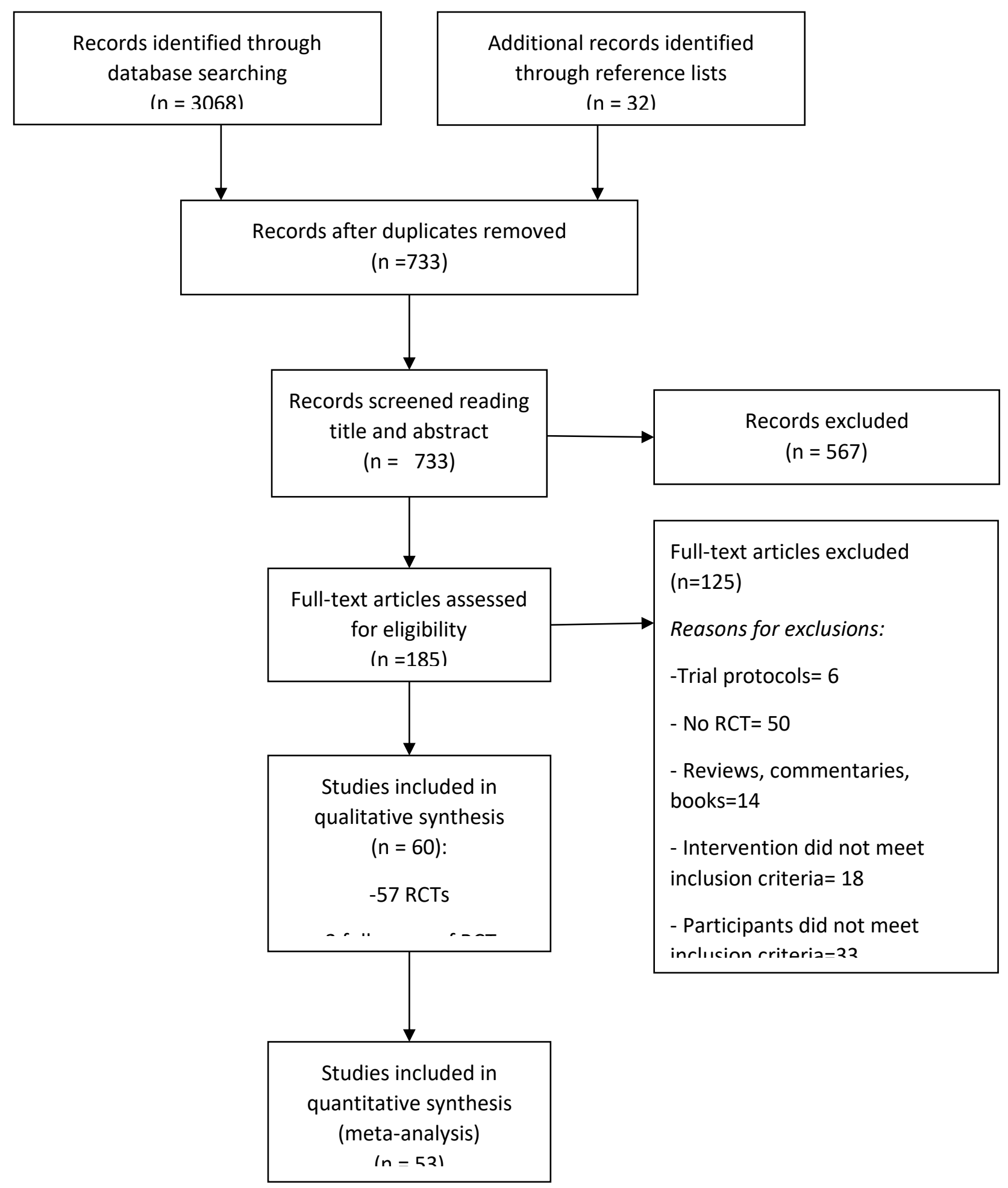

Figure 1. Flow diagram. 


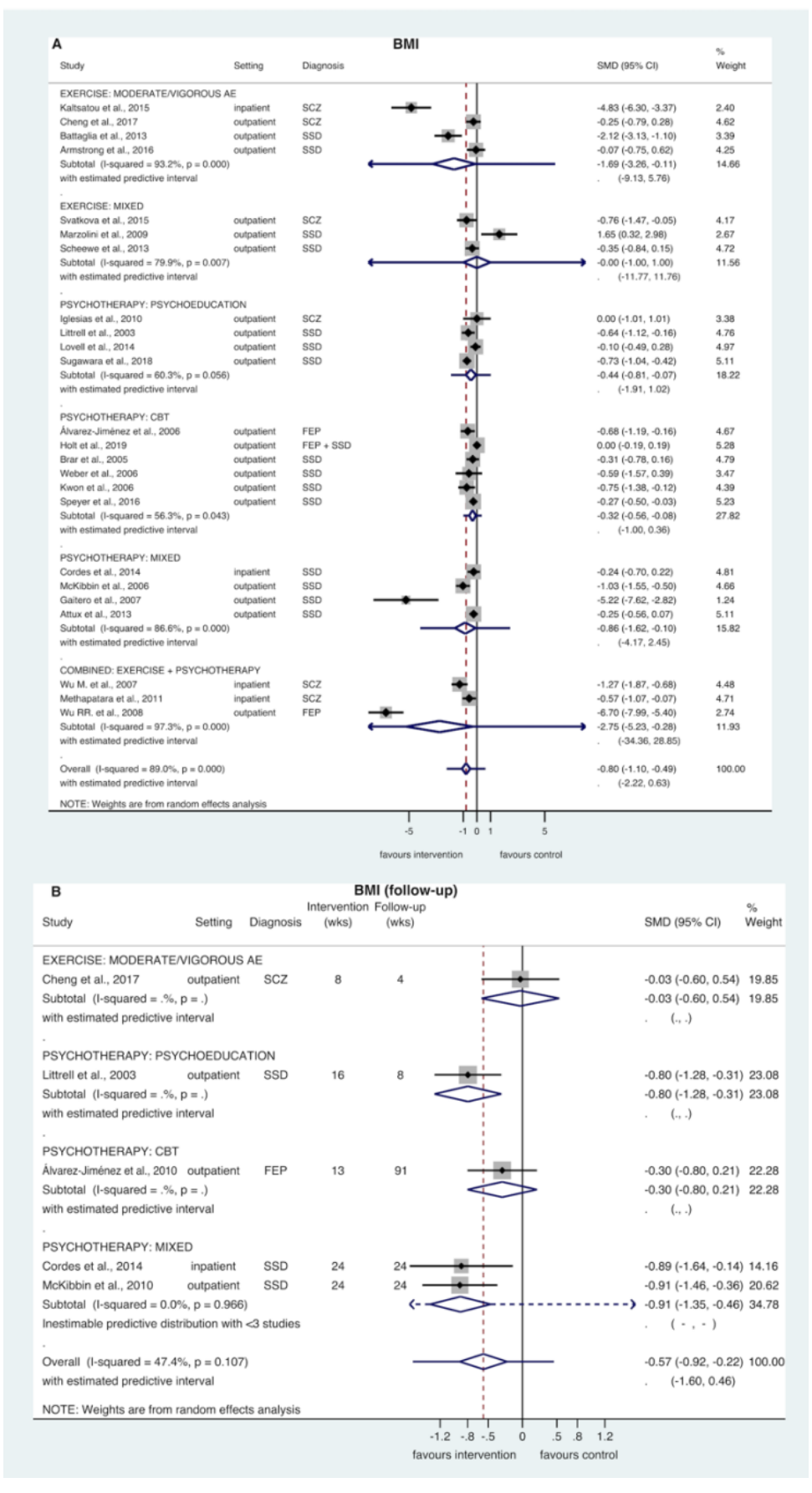

Figure 2. Forest plots for between-group (intervention vs control) difference in $\mathrm{BMI}$ change immediately after intervention (2A) or at the end of follow-up (2B). The effect size is expressed as standardized mean difference (Hedges' $\mathrm{g}$ ): a negative effect size indicates a relative decrease in BMI in the intervention group compared to controls (thus in favour of intervention), whereas a positive effect size indicates a relative BMI increase in the intervention group (thereby favouring controls). Effect sizes from individual studies are accompanied by their $95 \%$ confidence intervals (represented as back horizontal lines); the size of the grey boxes in the forest plots is proportional to the weight assigned to each study according to DerSimonian-Laird random-effects weighting. Meta-analysis was performed over all studies and within subgroups defined by subtype of intervention: the overall effect size is represented by a diamond, where its center (and point of maximum width) is located at average effect size, $95 \%$ confidence intervals for the average effect size are indicated by the ends of the diamond, and $95 \%$ predictive 
intervals are indicated by horizontal lines extending from each end of the diamond. The dashed vertical line indicates overall effect size. Abbreviations: BMI: body mass index; SMD: standardized mean difference; $\mathrm{Cl}$ : confidence intervals; FEP: first-episode psychosis; SCZ: schizophrenia; SSD: schizophrenia-spectrum disorder; wks: weeks; AE: aerobic exercise; CBT: cognitive behavioral therapy. 


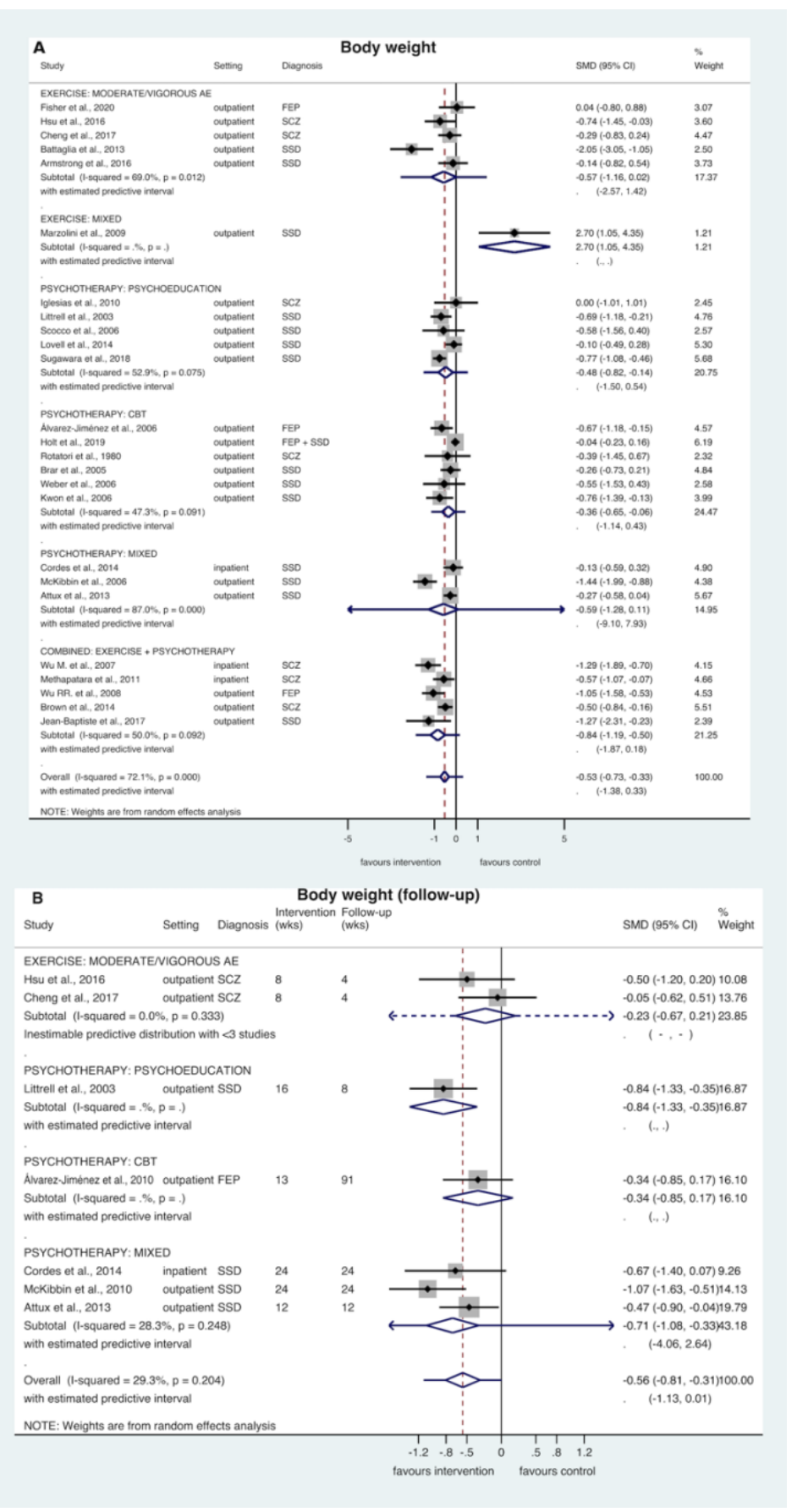

Figure 3. Forest plots for between-group (intervention vs control) difference in body weight change immediately after intervention (3A) or at the end of follow-up (3B). The effect size is expressed as standardized mean difference (Hedges' g): a negative effect size indicates a relative decrease in body weight in the intervention group compared to controls (thus in favour of intervention), whereas a positive effect size indicates a relative weight increase in the intervention group (thereby favouring controls). Effect sizes from individual studies are accompanied by their $95 \%$ confidence intervals (represented as back horizontal lines); the size of the grey boxes in the forest plots is proportional to the weight assigned to each study according to DerSimonian-Laird random-effects weighting. Meta-analysis was performed over all studies and within subgroups defined by subtype of intervention: the overall effect size is represented by a diamond, where its center (and point of maximum width) is located at average effect size, $95 \%$ confidence intervals for the average effect size are indicated by the ends of the 
diamond, and $95 \%$ predictive intervals are indicated by horizontal lines extending from each end of the diamond. The dashed vertical line indicates overall effect size. Abbreviations: SMD: standardized mean difference; $\mathrm{Cl}$ : confidence intervals; FEP: first-episode psychosis; SCZ: schizophrenia; SSD: schizophrenia-spectrum disorder; wks: weeks; AE: aerobic exercise; CBT: cognitive behavioral therapy. 


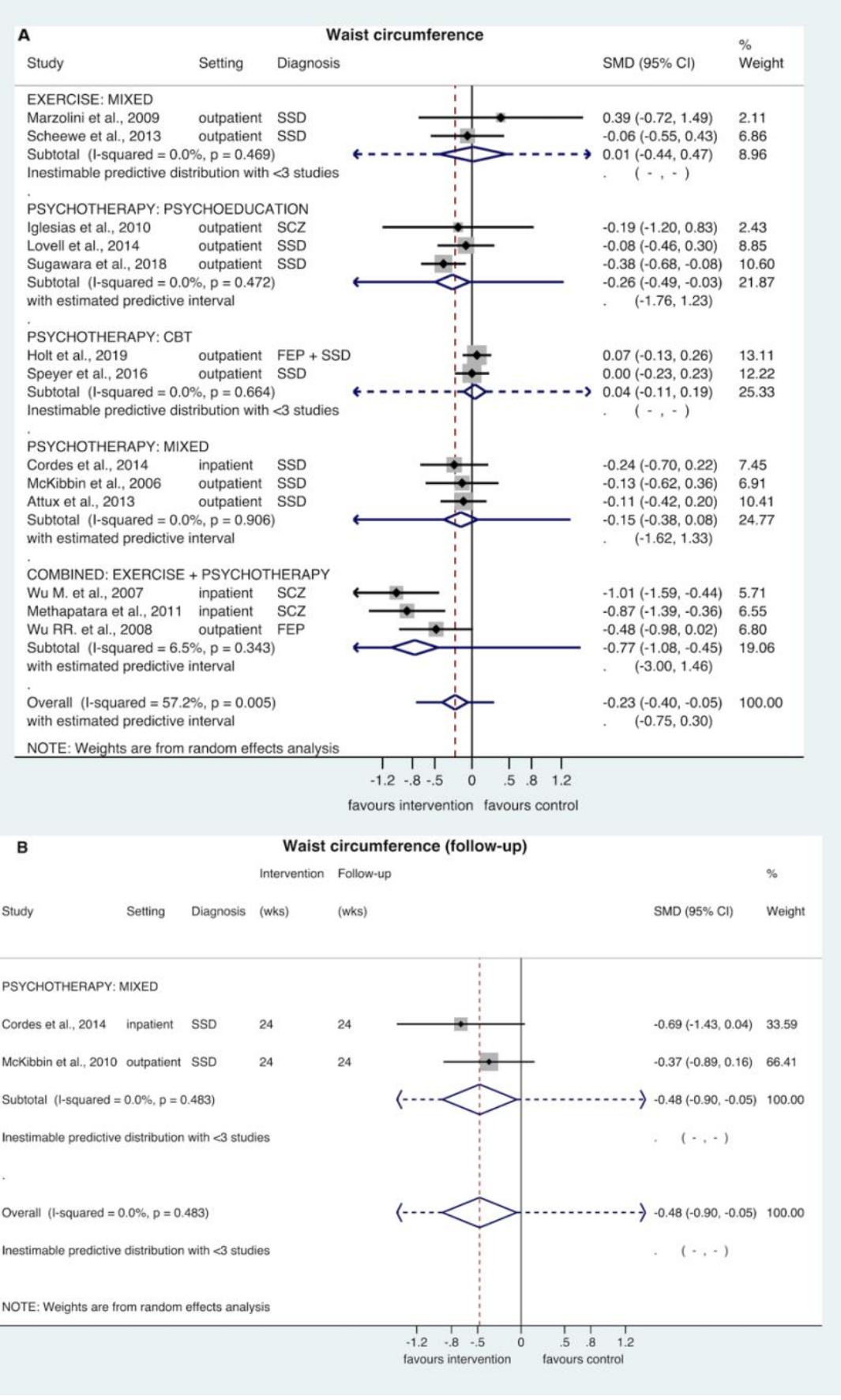

Figure 4. Forest plots for between-group (intervention vs control) difference in waist circumference change immediately after intervention (4A) or at the end of follow-up (4B). The effect size is expressed as standardized mean difference (Hedges' g): a negative effect size indicates a relative decrease in waist circumference in the intervention group compared to controls (thus in favour of intervention), whereas a positive effect size indicates a relative waist circumference increase in the intervention group (thereby favouring controls). Effect sizes from individual studies are accompanied by their 95\% confidence intervals (represented as back horizontal lines); the size of the grey boxes in the forest plots is proportional to the weight assigned to each study according to DerSimonian-Laird random-effects weighting. Meta-analysis was performed over all studies and within subgroups defined by subtype of intervention: the overall effect size is represented by a diamond, where its center (and point of maximum width) is located at average effect size, $95 \%$ confidence intervals for the average effect size are 
indicated by the ends of the diamond, and $95 \%$ predictive intervals are indicated by horizontal lines extending from each end of the diamond. The dashed vertical line indicates overall effect size. Abbreviations: SMD: standardized mean difference; $\mathrm{Cl}$ : confidence intervals; FEP: firstepisode psychosis; SCZ: schizophrenia; SSD: schizophrenia-spectrum disorder; wks: weeks; CBT: cognitive behavioral therapy. 


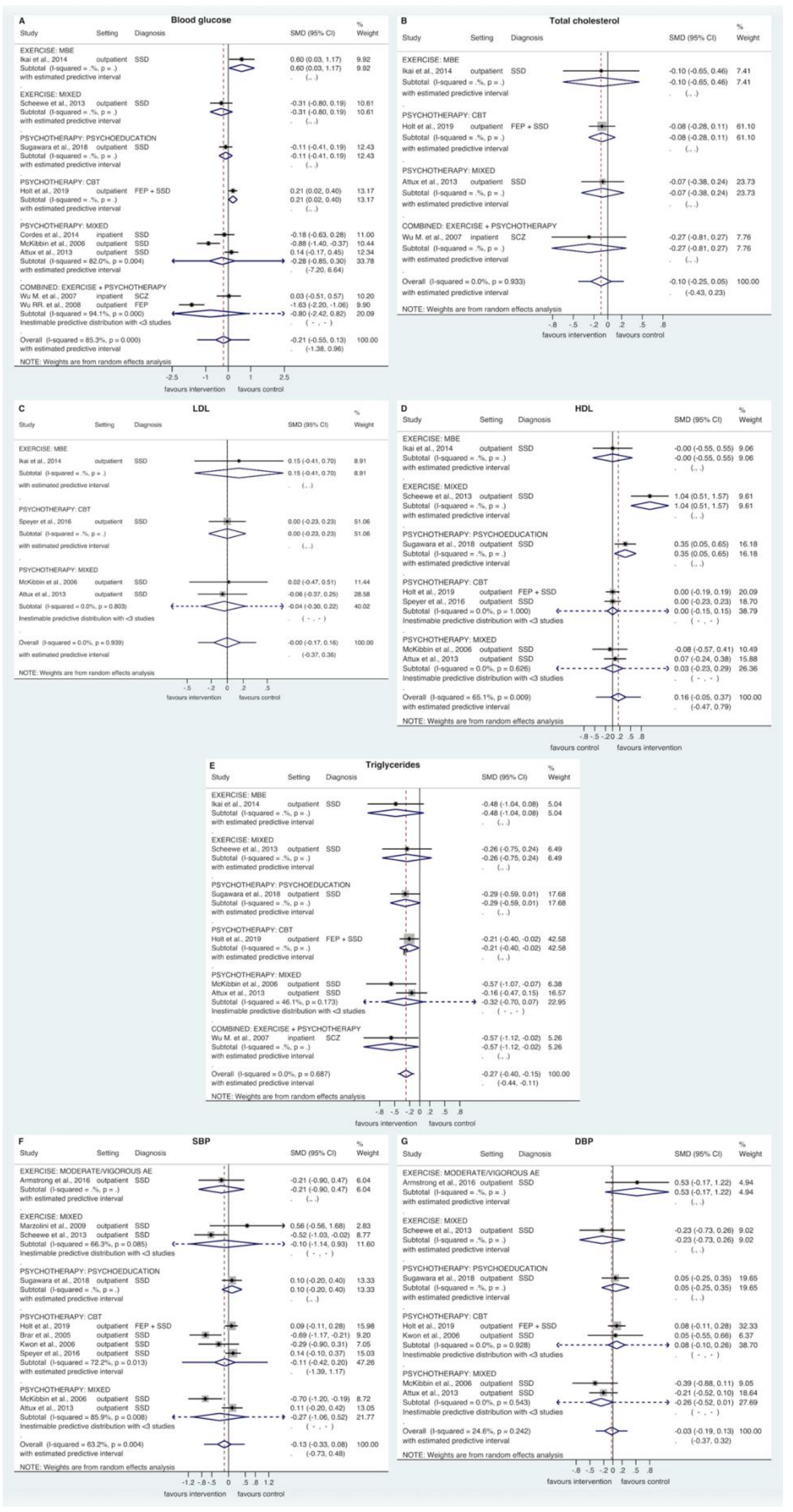


Figure 5. Forest plots for between-group (intervention vs control) difference in metabolic parameters change immediately after intervention: blood glucose (5A), total cholesterol (5B), LDL (5C), HDL (5D), triglycerides (5E), systolic blood pressure (5F) and diastolic blood pressure (5G). The effect size is expressed as standardized mean difference (Hedges' g): a negative effect size indicates a relative decrease in the intervention group compared to controls, whereas a positive effect size indicates the opposite. Thus, the sign of effect in favour of intervention will be different in each case (for instance, negative for LDL and positive for HDL); it is indicated at the bottom of each forest plot. Effect sizes from individual studies are accompanied by their $95 \%$ confidence intervals (represented as back horizontal lines); the size of the grey boxes in the forest plots is proportional to the weight assigned to each study according to DerSimonian-Laird random-effects weighting. Meta-analysis was performed over all studies and within subgroups defined by subtype of intervention: the overall effect size is represented by a diamond, where its center (and point of maximum width) is located at average effect size, $95 \%$ confidence intervals for the average effect size are indicated by the ends of the diamond, and $95 \%$ predictive intervals are indicated by horizontal lines extending from each end of the diamond. The dashed vertical line indicates overall effect size.

Abbreviations: LDL: low-density lipoprotein; HDL: high-density lipoprotein; SBP: systolic blood pressure; DBP: diastolic blood pressure; SMD: standardized mean difference; $\mathrm{Cl}$ : confidence intervals; FEP: first-episode psychosis; SCZ: schizophrenia; SSD: schizophrenia-spectrum disorder; wks: weeks; AE: aerobic exercise; MBE: mind-body exercise; CBT: cognitive behavioral therapy. 


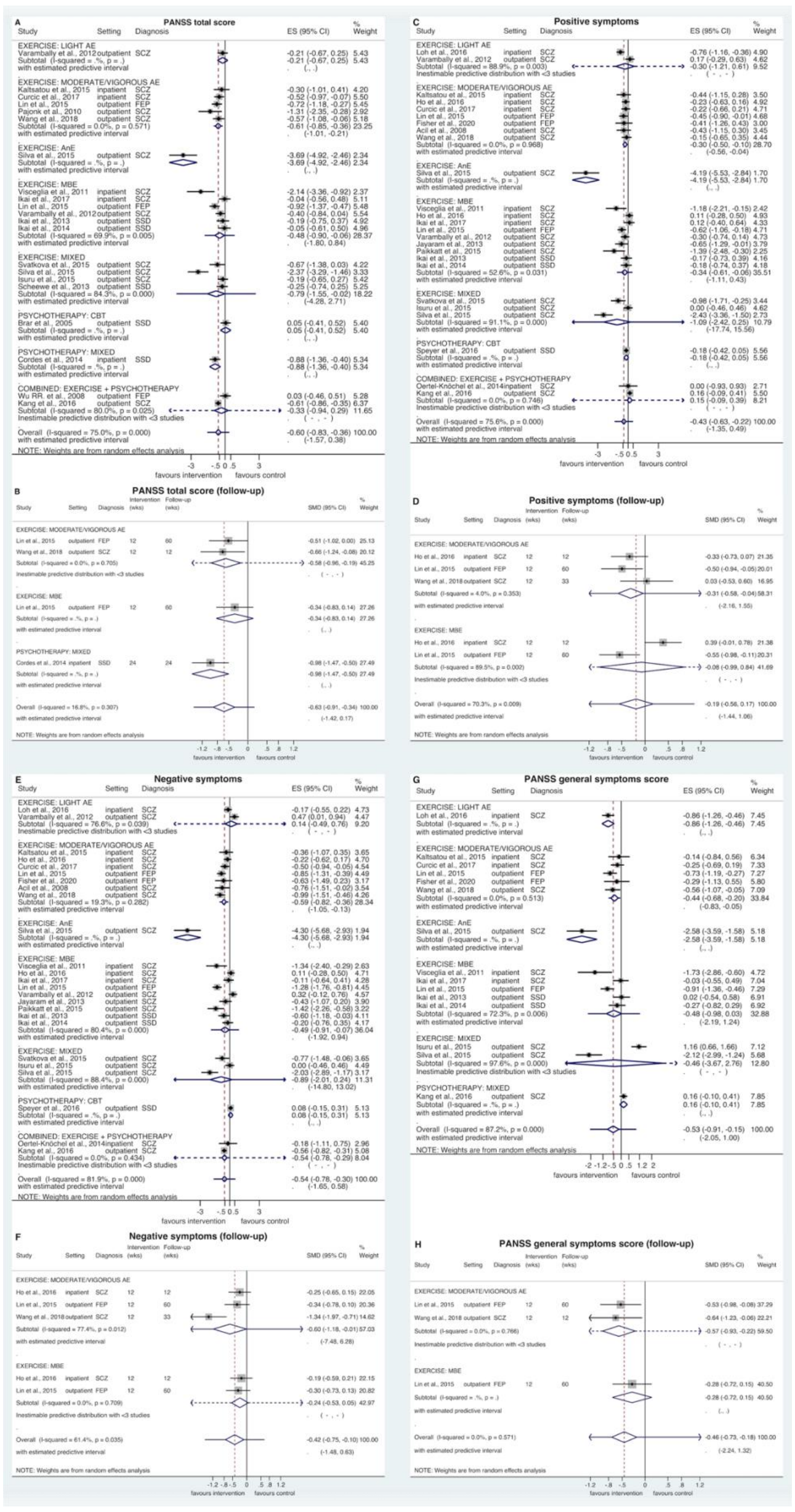


Figure 6. Forest plots for between-group (intervention vs control) difference in psychiatric symptoms change: PANSS total score (post-intervention: 6A; follow-up: 6B), positive symptoms (post-intervention: 6C; follow-up: 6D), negative symptoms (post-intervention: 6E; follow-up: 6F), PANSS general symptoms score (post-intervention: 6G; follow-up: 6H). The effect size is expressed as standardized mean difference (Hedges' g): a negative effect size indicates a relative decrease in the intervention group compared to controls (thus favouring intervention), whereas a positive effect size indicates the opposite. Effect sizes from individual studies are accompanied by their $95 \%$ confidence intervals (represented as back horizontal lines); the size of the grey boxes in the forest plots is proportional to the weight assigned to each study according to DerSimonian-Laird random-effects weighting. Meta-analysis was performed over all studies and within subgroups defined by subtype of intervention: the overall effect size is represented by a diamond, where its center (and point of maximum width) is located at average effect size, $95 \%$ confidence intervals for the average effect size are indicated by the ends of the diamond, and 95\% predictive intervals are indicated by horizontal lines extending from each end of the diamond. The dashed vertical line indicates overall effect size. Abbreviations: PANSS: Positive and Negative Syndrome Scale; SMD: standardized mean difference; $\mathrm{Cl}$ : confidence intervals; FEP: first-episode psychosis; SCZ: schizophrenia; SSD: schizophrenia-spectrum disorder; wks: weeks; AE: aerobic exercise; AnE: anaerobic exercise; $\mathrm{MBE}$ : mind-body exercise; $\mathrm{CBT}$ : cognitive behavioral therapy. 


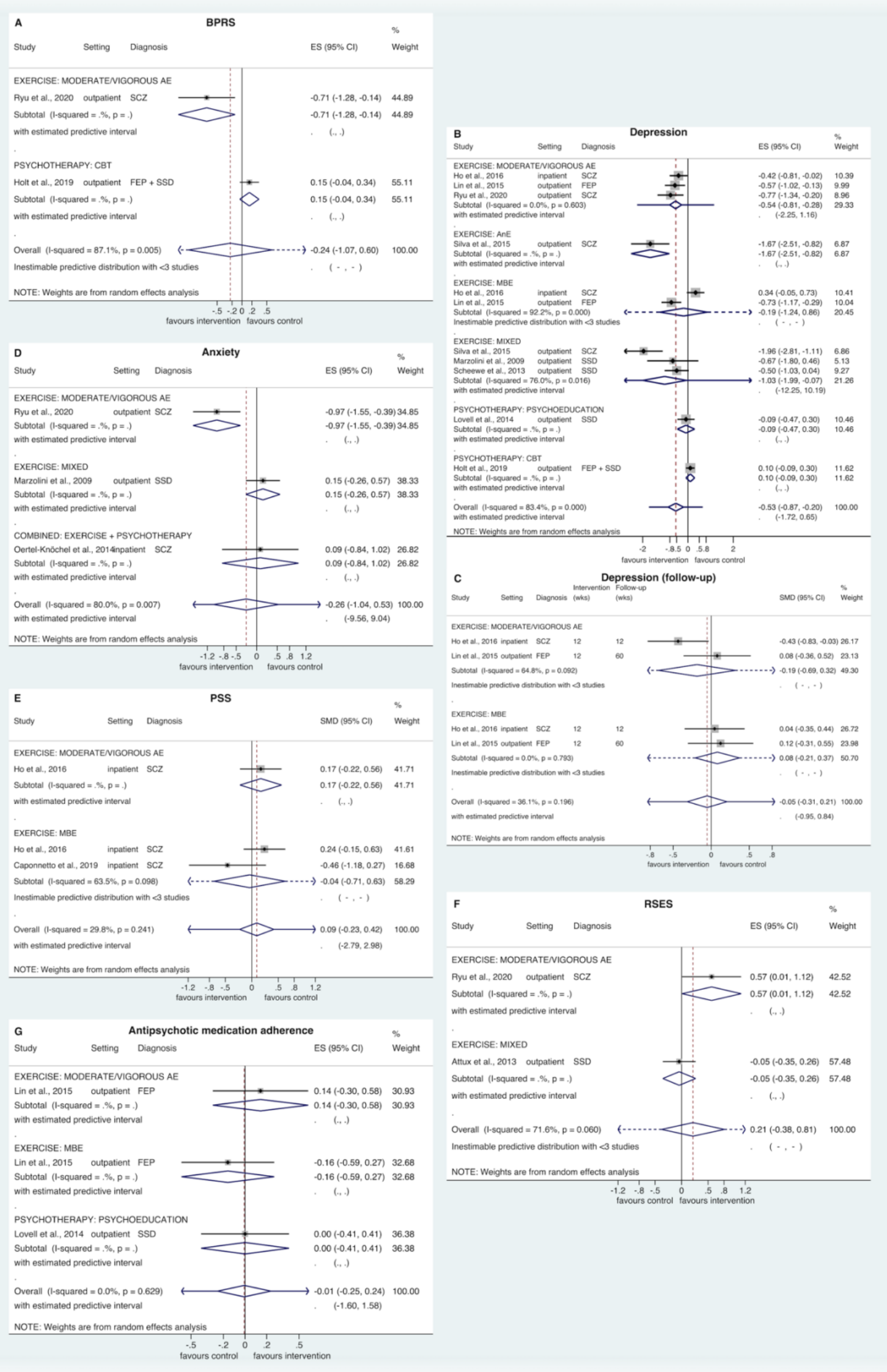

Figure 7. Forest plots for between-group (intervention vs control) difference in psychopathology change immediately after intervention (except for $\mathbf{7 C}$, which indicates change after follow-up): BPRS (7A), depressive symptoms (post-intervention: 7B; follow-up: 7C), state anxiety (7D), 
perceived stress scale (7E), Rosenberg Self-Esteem Scale (7F), antipsychotic medication adherence (7G). The effect size is expressed as standardized mean difference (Hedges' g): a negative effect size indicates a relative decrease in the intervention group compared to controls, whereas a positive effect size indicates the opposite; the sign of the effect in favour of intervention varies with the outcome and is indicated at the bottom of each plot. Effect sizes from individual studies are accompanied by their $95 \%$ confidence intervals (represented as back horizontal lines); the size of the grey boxes in the forest plots is proportional to the weight assigned to each study according to DerSimonian-Laird random-effects weighting. Meta-analysis was performed over all studies and within subgroups defined by subtype of intervention: the overall effect size is represented by a diamond, where its center (and point of maximum width) is located at average effect size, $95 \%$ confidence intervals for the average effect size are indicated by the ends of the diamond, and $95 \%$ predictive intervals are indicated by horizontal lines extending from each end of the diamond. The dashed vertical line indicates overall effect size. Abbreviations: BPRS: Brief Psychiatric Rating Scale; PSS: Perceived Stress Scale; RSES: Rosenberg Self-Esteem Scale; SMD: standardized mean difference; $\mathrm{Cl}$ : confidence intervals; FEP: first-episode psychosis; SCZ: schizophrenia; SSD: schizophrenia-spectrum disorder; wks: weeks; AE: aerobic exercise; AnE: anaerobic exercise; $\mathrm{MBE}$ : mind-body exercise; $\mathrm{CBT}$ : cognitive behavioral therapy. 


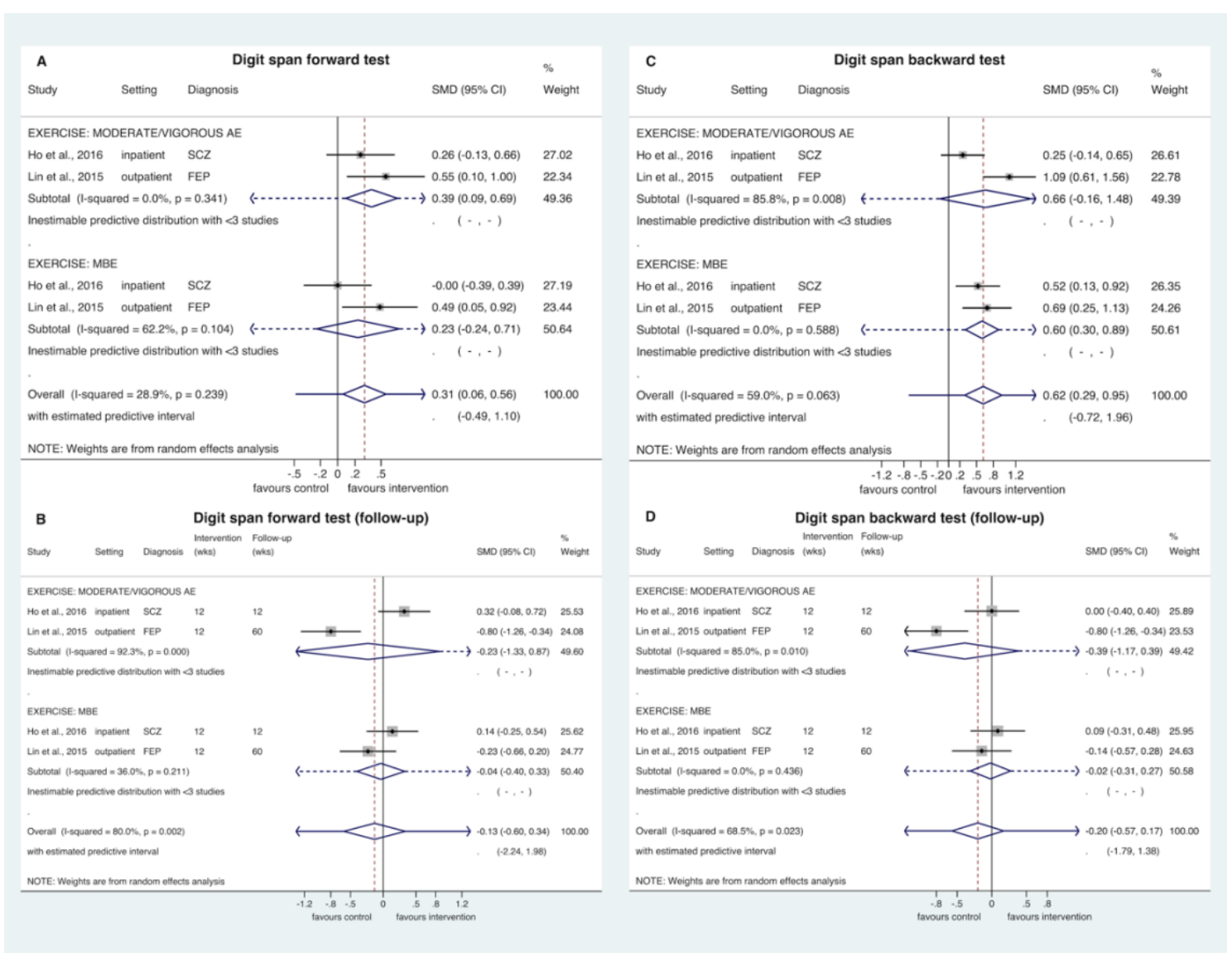

Figure 8. Forest plots for between-group (intervention vs control) difference in digit span test score change: forward score (post-intervention: 8A; follow-up: 8B); backward score (postintervention: 8C; follow-up: 8D). The effect size is expressed as standardized mean difference (Hedges' g): a positive effect size indicates a relative increase in the intervention group compared to controls (thus favouring intervention for these outcomes), whereas a negative effect size indicates the opposite. Effect sizes from individual studies are accompanied by their 95\% confidence intervals (represented as back horizontal lines); the size of the grey boxes in the forest plots is proportional to the weight assigned to each study according to DerSimonian-Laird random-effects weighting. Meta-analysis was performed over all studies and within subgroups defined by subtype of intervention: the overall effect size is represented by a diamond, where its center (and point of maximum width) is located at average effect size, 95\% confidence intervals for the average effect size are indicated by the ends of the diamond, and $95 \%$ predictive intervals are indicated by horizontal lines extending from each end of the diamond. The dashed vertical line indicates overall effect size. Abbreviations: SMD: standardized mean difference; $\mathrm{Cl}$ : confidence intervals; FEP: first-episode psychosis; SCZ: schizophrenia; SSD: schizophreniaspectrum disorder; wks: weeks; AE: aerobic exercise; AnE: anaerobic exercise; MBE: mind-body exercise; CBT: cognitive behavioral therapy. 


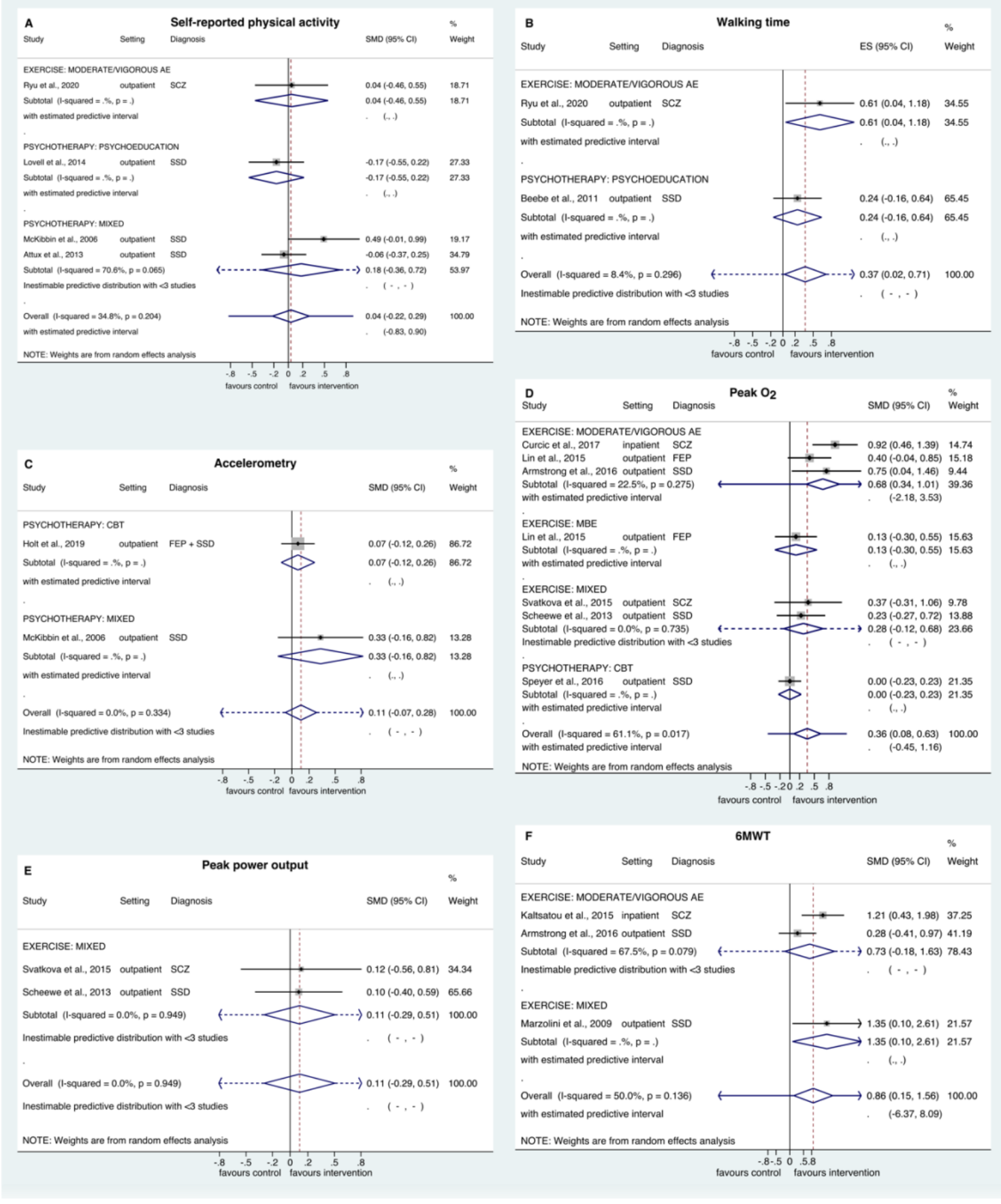

Figure 9. Forest plots for between-group (intervention vs control) difference in change in physical activity or performance immediately after intervention: self-reported physical activity (9A), walking time (9A), accelerometry (9C), peak $\mathrm{O}_{2}$ intake (9D), peak power output (9E) and $6 \mathrm{MWT}$ (9F). The effect size is expressed as standardized mean difference (Hedges' g): a positive effect size indicates a relative increase in the intervention group compared to controls (thus favouring intervention for these outcomes), whereas a negative effect size indicates the opposite. Effect sizes from individual studies are accompanied by their $95 \%$ confidence intervals (represented as back horizontal lines); the size of the grey boxes in the forest plots is proportional to the weight assigned to each study according to DerSimonian-Laird randomeffects weighting. Meta-analysis was performed over all studies and within subgroups defined by subtype of intervention: the overall effect size is represented by a diamond, where its center (and point of maximum width) is located at average effect size, $95 \%$ confidence intervals for the 
average effect size are indicated by the ends of the diamond, and 95\% predictive intervals are indicated by horizontal lines extending from each end of the diamond. The dashed vertical line indicates overall effect size. Abbreviations: 6MWT: 6-Minute Walking Test; SMD: standardized mean difference; $\mathrm{Cl}$ : confidence intervals; FEP: first-episode psychosis; SCZ: schizophrenia; SSD: schizophrenia-spectrum disorder; wks: weeks; AE: aerobic exercise; AnE: anaerobic exercise; MBE: mind-body exercise; CBT: cognitive behavioral therapy. 


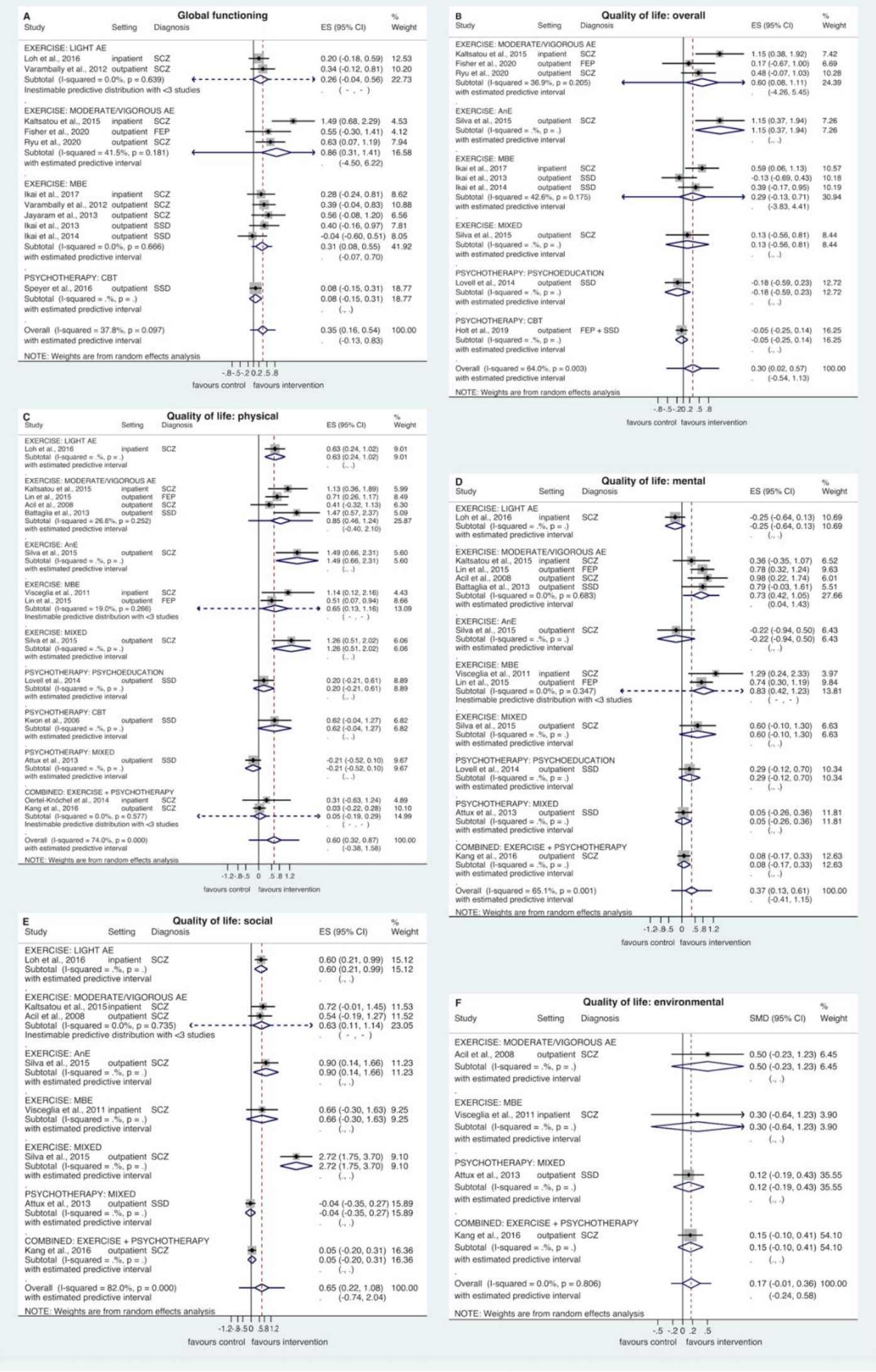

Figure 10. Forest plots for between-group (intervention vs control) difference in change in global functioning and quality of life immediately after intervention: global functioning (10A); quality of life: overall (10B), physical (10C), mental (10D), social (10E) and environmental (10F). The 
effect size is expressed as standardized mean difference (Hedges' g): a positive effect size indicates a relative increase in the intervention group compared to controls (thus favouring intervention for these outcomes), whereas a negative effect size indicates the opposite. Effect sizes from individual studies are accompanied by their $95 \%$ confidence intervals (represented as back horizontal lines); the size of the grey boxes in the forest plots is proportional to the weight assigned to each study according to DerSimonian-Laird random-effects weighting. Meta-analysis was performed over all studies and within subgroups defined by subtype of intervention: the overall effect size is represented by a diamond, where its center (and point of maximum width) is located at average effect size, 95\% confidence intervals for the average effect size are indicated by the ends of the diamond, and $95 \%$ predictive intervals are indicated by horizontal lines extending from each end of the diamond. The dashed vertical line indicates overall effect size. Abbreviations: SMD: standardized mean difference; $\mathrm{Cl}$ : confidence intervals; FEP: firstepisode psychosis; SCZ: schizophrenia; SSD: schizophrenia-spectrum disorder; wks: weeks; AE: aerobic exercise; AnE: anaerobic exercise; MBE: mind-body exercise; $\mathrm{CBT}$ : cognitive behavioral therapy. 